Japan. J. Math.

Vol. 8, No. 1, 1982

\title{
Contact geometry of higher order
}

\author{
By Keizo Yamaguchi \\ (Received April 14, 1981)
}

\section{Introduction}

Around 1900, E. Cartan initiated the study of the general equivalence problems of geometric structures and pseudo-groups by his theory of exterior differential systems. In this study he established the notion of involutive systems and the prolongation scheme for exterior differential systems. In [5] and [6], by his method of the equivalence, he dealt directly with involutive systems of second order partial differential equations of one unknown function with 2 or 3 independent variables. There he investigated the contact equivalence and the integration problem by ordinary differential equations of such involutive systems, following the tradition of the geometric theory of partial differential equations developed by Monge, Jacobi, Lie, Darboux, Goursat and others.

The rigorous formulation of involutive systems and the prolongation scheme for exterior differential systems was given by M. Kuranishi in [13] and [14]. Furthermore the algebraic aspect of the notion of involutiveness was clarified by V. W. Guillemin, S. Sternberg and J. P. Serre. Nowadays the involutive systems of non-linear partial differential equations are formulated in terms of Ehresmann's theory of jets as in [9] and [15]. However, in this formulation, it seems that the contact equivalence approach to involutive systems is missing. On the other hand the equivalence problem of (linear) differential systems, or more generally linear group structures subordinate to differential systems, was investigated by N. Tanaka. And he introduced the notion of graded algebras associated with a differential system and established a new prolongation scheme for such structures [19].

The main purpose of this paper is to give a rigorous formulation of the geometric aspects of [5] and [6]. More precisely we will clarify the notion of the "contact equivalence of involutive systems". For this purpose, starting from a contact manifold $\mathfrak{c}$, we first construct higher order prolongations

*) This research was partially supported by Grant-in-Aid for Scientific Research (No. 474003, 574004, 534001), Ministry of Education. 
$\left(J^{k}(\Subset), C^{k}\right)$ of $\Subset$ geometrically and clarify the notion of contact transformations of higher order. By this construction, we also clarify the geometric structure of Jet bundles, which leads us to the formulation of the notion of higher order contact manifolds. Furthermore, by analyzing the geometric structure which submanifolds of $J^{k}(\mathfrak{C})$ possess, we establish the notion of a $P D$ manifold. A PD manifold abstractly represents a system of partial differential equations of one unknown function having no equations of the first order and satisfying some regularity conditions. And the (local) equivalence of $\mathrm{PD}$ manifolds corresponds to the (local) contact equivalence of representing partial differential equations. Besides, the notion of symbol algebras of a PD manifold is introduced, in terms of which the involutiveness of the PD manifold is characterized. The symbol algebra of an involutive PD manifold expresses the structure formula of the representing involutive system in the sense of E. Cartan.

Now we proceed to the description of the contents of the following sections and explain main results of this paper. A differential system $D$ on a manifold $M$ is, by definition, a subbundle of the tangent bundle $T(M)$ of $M$. In $\S 1$, we fix our terminology for differential systems and related concepts, and prepare several lemmas for later use.

In $\S 2$, starting from a contact manifold $\Subset=(K, C)$ of dimension $2 n+1$, we first study the Lagrange-Grassmann bundle $(L(\mathbb{\complement}), E)$ over $\sqrt{5}$ and its higher prolongations $\left(J^{k}(\mathfrak{E}), C^{k}\right)(k \geqq 3)$. $\quad J^{k}(\mathfrak{S})$ is constructed as a submanifold of $J\left(J^{k-1}(\mathbb{E}), n\right)$ and $C^{k}$ is the canonical (differential) system on $J^{k}(\mathfrak{C})$, where $J\left(J^{k-1}(\mathfrak{5}), n\right)$ denotes the Grassmann bundle over $J^{k-1}(\mathfrak{5})$ consisting of all $n$-dimensional contact elements to $J^{k-1}(\mathfrak{C})$ (for the precise definitions see $\S 2)$. ( being regarded, by the Darboux's theorem, as the space of 1-jets of one unknown function, $\left(J^{k}(\mathcal{C}), C^{k}\right)$ corresponds to the space of $k$-jets, where we set $(L(\mathfrak{S}), E)=\left(J^{2}(\mathfrak{S}), C^{2}\right)$ by convention. Our emphasis is to be laid on the fact that $(L(\mathbb{E}), E)$ is a differential system with geometrically defined $n$ independent variables. And $\left(J^{k}(\Im), C^{k}\right)(k \geqq 3)$ are obtained as the prolongations of $(L(\sqrt{ }), E)$.

$\S 3$ is concerned with contact transformations of higher order. We first prove: Let $\widetilde{S}$ and $\hat{\mathbb{C}}$ be contact manifolds of dimension $2 n+1$ and let $\left(J^{k}(\mathfrak{S})\right.$, $\left.C^{k}\right)$ and $\left(J^{k}(\hat{\mathfrak{C}}), \hat{C}^{k}\right)$ be the $k$-th order prolongations of $\mathfrak{C}$ and $\hat{\mathbb{C}}$ respectively. A contact transformation $\phi$ of $\mathbb{E}$ onto $\hat{\mathbb{E}}$ induces a unique isomorphism $\psi=$ $q^{k} \dot{\phi}$ of $\left(J(\mathcal{E}), C^{k}\right)$ onto $\left(J^{k}(\hat{\mathbb{C}}), \hat{C}^{k}\right)$ and vice versa (Theorem 3.2). Although this theorem is, in its local form, substantially known in classical literature (cf. [2], [5] and [6]), it seems that our formulation reveals its geometric contents most naturally. Furthermore it is shown that $C^{k}$ is a regular differential system of type $\mathfrak{S}^{k}(n)$ on $J^{k}(\mathfrak{S})$ in the sense of N. Tanaka [19], where $\mathfrak{F}^{k}(n)$ is a fundamental graded algebra of $(k+1)$-th kind and is called 
the contact algebra of order $k$ of degree $n$ (see Definition 3.5). And we study the structure of ${ }^{k}(n)$ in detail. The fundamental identification and the affine bundle structure of Jet bundles are formulated in our geometric setting by means of graded algebras of $\left(J^{k}(ङ), C^{k}\right)$ (see also $\S 6$ ).

In $\S 4$, we study submanifolds $R$ of $J^{k}(\mathfrak{S})$ and investigate the geometric structure of $R$ which is inherited from $\left(J^{k}(\mathfrak{C}), C^{k}\right)$. More precisely let $\iota$ be the inclusion of $R$ into $J^{k}(\mathfrak{S})$ and set $\rho_{r}=\iota \cdot \rho_{r}^{k}$, where $\rho_{r}^{k}$ is the projection of $J^{k}(\mathfrak{S})$ onto $J^{r}(\mathfrak{S})$. Assume that $D^{r}=\left(\rho_{r}\right)_{*}^{-1}\left(C^{r}\right)$ is a differential system on $R$ for $r=1, \cdots, k$. In this case $\left(D^{1}, \cdots, D^{k}\right)$ is called the contact system of order $k$ associated with $R$. We study the properties of $\left(D^{1}, \cdots, D^{k}\right)$ in detail. And involutive submanifolds are characterized in terms of graded subalgebras associated with $R$. Furthermore the contact equivalence of involutive submanifolds is discussed.

We start our study towards the geometrization of involutive systems in $\S 5$. In view of the results obtained in $\S 4$, we first introduce the notion of a PD manifold (Definition 5.1):

$A P D$ manifold $\left(R ; D^{1}, \cdots, D^{k}\right)$ of order $k$ of degree $n$ consists of a manifold $R$ together with a family $\left\{D^{1}, \cdots, D^{k}\right\}$ of differential systems on $R$ satisfying the following conditions.

(1) $D^{1}$ and $D^{2}$ are differential systems of codimension 1 and $n+1$ respectively.

(2) The derived space $\partial D^{r+1}(x)$ of $D^{r+1}$ at $x$ is a subspace of $D^{r}(x)$ at each $x \in R$ for $r=1, \cdots, k-1$.

(3) The assignment $x \mapsto F^{r}(x)=C h\left(D^{1}\right)(x) \cap \cdots \cap C h\left(D^{r}\right)(x)$ defines a subbundle $F^{r}$ of $D^{r+1}$ of codimension $n$ for $r=1, \cdots, k-1$, where $C h\left(D^{i}\right)(x)$ is the Cauchy-Cartan characteristic subspace of $D^{i}$ at $x \in R$.

(4) $\quad F^{k}(x)=C h\left(D^{1}\right)(x) \cap \cdots \cap C h\left(D^{k}\right)(x)=\{0\} \quad$ at each $x \in R$.

(5) If $k \geqq 3, F^{k-1}=F^{1} \cap D^{k}$.

By utilizing the Realization Lemma (Lemma 1.5), we establish the Realization Theorem (Theorem 5.3) for a PD manifold of order $k$ of degree $n$, which says that $\left(R ; D^{1}, \cdots, D^{k}\right)$ is locally realized as a submanifold of $J^{k}($ () in a canonical manner such that $\left(D^{1}, \cdots, D^{k}\right)$ coincides with the associated contact system of order $k$, where $\sqrt{5}$ is, roughly speaking, the contact manifold of dimension $2 n+1$ obtained as the quotient manifold of $R$ by the foliation defined by $F^{1}=C h\left(D^{1}\right)$ (for the precise statement of Theorem 5.3, see $\S 5)$. Furthermore an isomorphism of $\mathrm{PD}$ manifolds is compatible with the above realization as submanifolds of $J^{k}(\sqrt{ })$ (Corollary 5.4). Thus a PD manifold of order $k$ of degree $n$ abstractly represents (locally) a system of $k$-th order partial differential equations of one unknown function with $n$ independent variables having no equations of the first order. And the (local) 
equivalence of PD manifolds corresponds to the (local) contact equivalence of representing partial differential equations.

We next introduce the notion of symbol algebras of a PD manifold, which is an analogue of graded algebras of a regular differential system. Let $x$ be any point of $R$. We set

$$
g(x)=\sum_{p=-1}^{-(k+1)} g_{p}(x) \quad \text { (direct sum), }
$$

where $\mathfrak{\Xi}_{-(k+1)}(x)=T_{x}(R) / D^{1}(x), \mathfrak{马}_{p}(x)=D^{p+k+1}(x) / D^{p+k+2}(x) \quad(-k \leqq p \leqq-2)$ and $\mathfrak{Z}_{-1}(x)=D^{k}(x)$. Then, by (2) of Definition 5.1, it is shown that $\mathfrak{g}(x)$ is endowed with a structure of graded Lie algebra (see 1.3 of $\S 1)$. $\xi(x)$ is called the symbol algebra of $\left(R ; D^{1}, \cdots, D^{k}\right)$ at $x$ (Definition 5.5). We study the structure of $\mathfrak{s}(x)$ in detail. In particular it is shown that there exists a graded Lie algebra isomorphism of $\mathfrak{z}(x)$ into $\mathfrak{S}^{k}(n)$ and that two such isomorphisms $\phi_{1}$ and $\phi_{2}$ differ by an automorphism $A$ of $\Im^{k}(n)$, i.e., $\phi_{1}=A \cdot \phi_{2}$. The involutiveness of a PD manifold is formulated in terms of symbol algebras. Furthermore the first prolongation $R^{(1)}$ of an involutive PD manifold $\left(R ; D^{1}\right.$, $\left.\cdots, D^{k}\right)$ is shown to have the structure of an involutive PD manifold of order $k+1$ of degree $n$. And the affine bundle structure of $R^{(1)}$ is explained by means of symbol algebras of $R$.

In $\S 6$, we formulate contact manifolds of higher order (Definition 6.1):

Let $C$ be a differential system on a manifold $K$. Then $(K, C)$ is called a contact manifold of order $k$ of degree $n$ if and only if the following conditions are satisfied.

(1) There exists a family $\left\{C^{1}, \cdots, C^{k}\right\}$ of differential systems on $K$ such that $C^{k}=C$ and $C^{r}=\partial C^{r+1}$ for $r=1, \cdots, k-1$, i.e., the $r$-th derived system $\partial^{r} C$ of $C$ is a differential system on $K$ for $r=1, \cdots, k-1$.

(2) $C^{1}$ is a differential system of codimension 1.

(3) $C^{2}$ is a differential system of codimension $n+1$.

(4) The Cauchy-Cartan characteristic system $C h\left(C^{r}\right)$ of $C^{r}$ is a subbundle of $C^{r+1}$ of codimension $n$ for $r=1, \cdots, k-1$.

(5) $\quad C h\left(C^{k}\right)(x)=\{0\} \quad$ at each $x \in K$.

(6) $\quad C h\left(C^{k-1}\right)=C h\left(C^{1}\right) \cap C^{k}(k \geqq 3)$.

(7) $\operatorname{dim} K=\sum_{r=1}^{k}{ }_{n} H_{r}+n+1$, where ${ }_{n} H_{r}=\left(\begin{array}{c}n+r-1 \\ r\end{array}\right)$.

Let $(K, C)$ be a contact manifold of order $k$ of degree $n$. Then it is shown that $(K, C)$ is locally isomorphic with $\left(J^{k}(\mathfrak{\complement}), C^{k}\right)$, where $\mathfrak{S}$ is a contact manifold of dimension $2 n+1$. Furthermore we characterize $(K, C)$ in terms of graded algebras.

Finally $\S 7$ is concerned with the characteristics in the sense of Monge. 
Furthermore we discuss their relation with covariant systems of $\left(R ; D^{1}, \cdots\right.$, $\left.D^{k}\right)$.

The author is grateful to Professor N. Tanaka for invaluable discussions during the course of preparation of this paper. In particular, our present formulation of a PD manifold is inspired by his recent work [20] (cf. Lemma 1.5).

Throughout this paper we always assume the differentiability of class $C^{\infty}$, though the argument goes through in real or complex analytic category with suitable modifications.

\section{Table of Contents}

$\S 1$. Preliminaries on differential systems

1.1. Differential systems.

1.2. Derived systems.

1.3. Graded algebras associated with differential systems.

1.4. Cauchy-Cartan characteristic systems.

1.5. Canonical systems on Grassmann bundles.

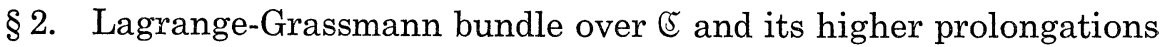

2.1. Lagrange-Grassmann bundle $L(\mathbb{C})$.

2.2. Higher prolongations $\left(J^{k}(\mathfrak{S}), C^{k}\right)$.

2.3. Canonical system $C^{k}$ on $J^{k}(\mathbb{E})$.

§ 3. Contact transformations and the symbol algebra of $\left(J^{k}(\mathfrak{S}), C^{k}\right)$

3.1. Contact transformations.

3.2. Symbol algebra of $\left(J^{k}(\mathfrak{S}), C^{k}\right)$.

3.3. Fundamental identification of $Q^{k}$.

§4. Submanifolds of $\left(J^{k}(\mathbb{E}), C^{k}\right)$

4.1. Submanifolds of $\left(J^{k}(\mathfrak{S}), C^{k}\right)$.

4.2. Involutive submanifolds.

4.3. Contact equivalence of involutive submanifolds.

§5. Geometrization of involutive systems

5.1. Contact system of order $k$.

5.2. Symbol algebra of a PD manifold.

5.3. Involutive $\mathrm{PD}$ manifolds.

$\S 6$. Higher order contact manifolds

6.1. Higher order contact manifolds.

6.2. Characterization by graded algebras. 
$\S 7 . \quad$ Monge characteristics and Covariant systems

7.1. Polar subspaces and Characteristic ideal $\mathfrak{M}(f(x))$.

7.2. Characteristic elements.

7.3. Covariant systems of regularly involutive PD manifolds.

\section{$\S 1$. Preliminaries on differential systems}

In this section we will recall the basic concepts and fix our terminology for differential systems, partially following [6], [18] and [19]. And further, we will give several lemmas for later use.

1.1. Differential systems. Let $M$ be a manifold of dimension $m$. Let $T(M)$ and $T^{*}(M)$ denote the tangent and the cotangent bundle of $M$ respectively. A differential system $D$ on $M$ of rank $r$ is, by definition, a subbundle of $T(M)$ of fibre dimension $r$. Let $D(x)$ denote the fibre of $D$ at $x \in M$. $D$ is also defined by the annihilator subbundle $D^{\perp}$ of $T^{*}\left(M^{*}\right)$ of fibre dimension $m-r$, where

$$
D^{\perp}(x)=\left\{\omega \in T_{x}^{*}(M) \mid \omega(X)=0 \text { for any } X \in D(x)\right\} .
$$

In this context $D$ is called a differential system of codimension $m-r$ and $D^{\perp}$ is called the annihilator bundle of $D$. Let $\omega_{1}, \cdots, \omega_{m-r}$ be 1 -forms defined on a neighborhood $U$ of $x \in M$ such that $\left(\omega_{1}\right)_{y}, \cdots,\left(\omega_{m-r}\right)_{y}$ form a basis of $D^{\perp}(y)$ at each $y \in U$. Then we say that $D$ is defined on $U$ by $\omega_{1}, \cdots, \omega_{m-r}$.

Let $M$ and $\hat{M}$ be manifolds of dimension $m$. Let $D_{i}$ and $\hat{D}_{i}(i=1, \cdots, s)$ be families of differential systems on $M$ and $\hat{M}$ respectively. A diffeomorphism $\phi$ of $M$ onto $\hat{M}$ is called an isomorphism of $\left(M ; D_{1}, \cdots, D_{s}\right)$ onto $\left(\hat{M} ; \hat{D}_{1}, \cdots, \hat{D}_{s}\right)$ if $\phi_{*}\left(D_{i}(x)\right)=\hat{D}_{i}(\phi(x))$ for each $x \in M$ and $i=1, \cdots, s$.

Let $(M, N, p)$ be a fibred manifold, i.e., $p$ is a submersion of $M$ onto $N$. Let $D$ be a differential system on $N$ and let $x$ be any point of $M$. We denote by $p_{*}^{-1}(D)$ and $\operatorname{Ker} p_{*}$ the differential systems on $M$ defined by

$$
\begin{aligned}
& p_{*}^{-1}(D)(x)=\left\{X \in T_{x}(M) \mid p_{*}(X) \in D(p(x))\right\}, \\
& \operatorname{Ker} p_{*}(x)=\left\{X \in T_{x}(M) \mid p_{*}(X)=0\right\} .
\end{aligned}
$$

More generally, let $\phi$ be a differentiable map of $M$ into $N$. If $\operatorname{dim} \phi_{*}^{-1}(D(\phi(x)))$ is constant on $M$, then it is easy to see that the assignment $x \mapsto \phi_{*}^{-1}(D(\phi(x)))$ defines a differential system on $M$. In this case we denote this differential system also by $\phi_{*}^{-1}(D)$.

Let $\mathscr{R}$ be the structure sheaf of $M$, i.e., the sheaf of germs of $C^{\infty}$-functions of $M . \mathscr{R}$ is a sheaf of rings. And let $\mathscr{T}$ and $\mathscr{T}^{*}$ denote the sheaves of germs of local sections of $T(M)$ and $T^{*}(M)$ respectively. $\mathscr{T}$ and $\mathscr{T}^{*}$ are sheaves of $\mathscr{R}$-modules, and $\mathscr{T}$ is also a sheaf of Lie algebras. For a differ- 
ential system $D$ on $M$, we denote the sheaf of germs of local sections of $D$ (resp. $D^{\perp}$ ) by the corresponding script letter $\mathscr{D}$ (resp. $\mathscr{D}^{\perp}$ ). And we call $\mathscr{D}$ (resp. $\mathscr{D}^{\perp}$ ) the sheaf of $D$ (resp. $D^{\perp}$ ). $\mathscr{D}$ (resp. $\mathscr{D}^{\perp}$ ) is a subsheaf of $\mathscr{R}$-modules of $\mathscr{T}$ (resp. $\left.\mathscr{T}^{*}\right)$. For a sheaf $\mathscr{S}$ on $M$, let $\Gamma(U, \mathscr{S})=\mathscr{S}(U)$ denote the sections of $\mathscr{S}$ on an open subset $U$ of $M$, and let $\mathscr{S}_{x}$ denote the stalk of $\mathscr{S}$ at $x \in M$.

1.2. Derived systems. Let $D$ be a differential system on $M$. First we will define the derived sheaf $\partial \mathscr{D}$ of $D$. For each open subset $U$ of $M, \mathscr{D}(U)$ is an $\mathscr{R}(U)$-submodule of $\mathscr{T}(U)$, hence is a linear subspace of $\mathscr{T}(U)$. Let $[\mathscr{D}(U), \mathscr{D}(U)]$ denote the linear subspace of $\mathscr{T}(U)$ generated by the set of elements $[X, Y]$, where $X, Y \in \mathscr{D}(U)$. Let $\check{\mathscr{D}}(U)$ be the linear subspace of $\mathscr{T}(U)$ spanned by $\mathscr{D}(U)$ and $[\mathscr{D}(U), \mathscr{D}(U)]$, i.e.,

$$
\check{\mathscr{D}}(U)=\mathscr{D}(U)+[\mathscr{D}(U), \mathscr{D}(U)] .
$$

Then it is easy to see that $\check{\mathscr{D}}(U)$ is an $\mathscr{R}(U)$-submodule of $\mathscr{T}(U)$ and that the assignment $U \mapsto \check{D}(U)$ defines a presheaf on $M$. The derived sheaf $\partial \mathscr{D}$ of $D$ is, by definition, the sheaf generated by the presheaf $U \mapsto \check{\mathscr{D}}(U)$. $\partial \mathscr{D}$ is a subsheaf of $\mathscr{R}$-modules of $\mathscr{T}$ and the stalk $\partial \mathscr{D}_{x}$ of $\partial \mathscr{D}$ at $x \in M$ is given by

$$
\partial \mathscr{D}_{x}=\mathscr{D}_{x}+\left[\mathscr{D}_{x}, \mathscr{D}_{x}\right] \text {, }
$$

where [,] is the bracket operation of $\mathscr{T}_{x}$. Since $\partial \mathscr{D}$ is a subsheaf of $\mathscr{R}$ modules of $\mathscr{T}$, we can define the linear subspace $\partial D(x)$ of $T_{x}(M)$ by

$$
\partial D(x)=\left\{X_{x} \in T_{x}(M) \mid X \in \partial \mathscr{D}_{x}\right\},
$$

where $X_{x}$ denotes the tangent vector of $X$ at $x \in M$. As for $\partial D(x)$, we have

Lemma 1.1. $(\partial D(x))^{\perp}=\left\{\omega_{x} \in D^{\perp}(x) \mid \omega \in \mathscr{D}_{x}^{\perp},(d \omega)_{x} \equiv 0\left(\bmod D^{\perp}(x)\right)\right\}$, where $\omega_{x}$ denotes the cotangent vector of $\omega$ at $x \in M$.

Proof. Let $\omega, \omega^{\prime} \in \mathscr{D}_{x}^{\perp}$. It is easy to see that if $\omega_{x}=\omega_{x}^{\prime}$, then $(\mathrm{d} \omega)_{x} \equiv\left(\mathrm{d} \omega^{\prime}\right)_{x}$ $\left(\bmod D^{\perp}(x)\right)$. On the other hand any element of $D^{\perp}(x)$ can be extended to a local section of $D^{\perp}$. Hence $(\mathrm{d} \omega)_{x} \equiv 0\left(\bmod D^{\perp}(x)\right)$ is a condition for the cotangent vector $\omega_{x} \in D^{\perp}(x)$. Let $\omega$ be any element of $\mathscr{D} \frac{\perp}{x}$ such that $\omega_{x} \in(\partial D(x))^{\perp}$. Then, for $X, Y \in \mathscr{D}_{x},(\mathrm{~d} \omega)_{x}(X, Y)=-\omega_{x}([X, Y])=0$. Hence we have $\left.(\mathrm{d} \omega)_{x}\right|_{D(x)}$ $=0$, i.e., $(\mathrm{d} \omega)_{x} \equiv 0\left(\bmod D^{\perp}(x)\right)$.

Conversely, let $\omega$ be any element of $\mathscr{D} \frac{\perp}{x}$ such that $(\mathrm{d} \omega)_{x} \equiv 0\left(\bmod D^{\perp}(x)\right)$. Then, for $X, Y \in \mathscr{D}_{x}$, we have $\omega_{x}(X)=0$ and $\omega_{x}([X, Y])=-(\mathrm{d} \omega)_{x}(X, Y)=0$. Since $\partial D(x)$ is spanned by the vectors $X_{x}$ and $[X, Y]_{x}, X, Y \in \mathscr{D}_{x}$, we get $\omega_{x} \in$ $(\partial D(x))^{\perp}$.

q.e.d.

If $\operatorname{dim} \partial D(x)$ is constant on $M$, then the assignment $x \mapsto \partial D(x)$ defines the differential system $\partial D$ on $M$ such that $\partial \mathscr{D}$ coincides with the sheaf of $\partial D$. 
In this case we say that $\partial \mathscr{D}$ defines a differential system. $\partial D$ is called the (first) derived system of $D$.

Let $\partial \mathscr{D}^{\perp}$ be the subsheaf of $\mathscr{R}$-modules of $\mathscr{D}^{\perp}$ generated by the presheaf: $U \mapsto \check{D}^{\perp}(U)=\left\{\omega \in \mathscr{D}^{\perp}(U) \mid d \omega \equiv 0\left(\bmod \mathscr{D}^{\perp}(U)\right)\right\}$. And we set

$$
\partial D^{\perp}(x)=\left\{\omega_{x} \in T_{x}^{*}(M) \mid \omega \in \partial \mathscr{D}_{x}^{\perp}\right\} .
$$

In general we have $\partial D^{\perp}(x) \subset(\partial D(x))^{\perp}$. One should note that if $\operatorname{dim} \partial D(x)$ is constant on $M$, then $\partial D^{\perp}(x)$ coincides with $(\partial D(x))^{\perp}$ at each $x \in M$ and that, in this case, $\partial \mathscr{D}^{\perp}$ coincides with the sheaf of the annihilator bundle $\partial D^{\perp}$ of $\partial D$.

Now, for an integer $k \geqq 2$, we will define the $k$-th derived sheaf $\partial^{k} \mathscr{D}$ of $D$ and the $k$-th weak derived sheaf $\partial^{(k)} \mathscr{D}$ of $D$ inductively as follows. By convention we set $\partial \mathscr{D}=\partial^{1} \mathscr{D}=\partial^{(1)} \mathscr{D} . \quad \partial^{k-1} \mathscr{D}$ being defined $(k \geqq 2)$, the $k$-th derived sheaf $\partial^{k} \mathscr{D}$ is defined as the sheaf generated by the presheaf: $U \mapsto \check{\mathscr{D}}^{k}(U)$, where

$$
\check{\mathscr{D}}^{k}(U)=\partial^{k-1} \mathscr{D}(U)+\left[\partial^{k-1} \mathscr{D}(U), \partial^{k-1} \mathscr{D}(U)\right] .
$$

And $\partial^{(k-1)} \mathscr{D}$ being defined $(k \geqq 2)$, the $k$-th weak derived sheaf $\partial^{(k)} \mathscr{D}$ is defined as the sheaf generated by the presheaf: $U \mapsto \check{\mathscr{D}}^{(k)}(U)$, where

$$
\check{\mathscr{D}}^{(k)}(U)=\partial^{(k-1)} \mathscr{D}(U)+\left[\partial^{(k-1)} \mathscr{D}(U), \mathscr{D}(U)\right] .
$$

$\partial^{k} \mathscr{D}$ and $\partial^{(k)} \mathscr{D}(k \geqq 2)$ are subsheaves of $\mathscr{R}$-modules of $\mathscr{T}$ and their stalks at $x \in M$ are given by

$$
\begin{aligned}
& \partial^{k} \mathscr{D}_{x}=\partial^{k-1} \mathscr{D}_{x}+\left[\partial^{k-1} \mathscr{D}_{x}, \partial^{k-1} \mathscr{D}_{x}\right], \\
& \partial^{(k)} \mathscr{D}_{x}=\partial^{(k-1)} \mathscr{D}_{x}+\left[\partial^{(k-1)} \mathscr{D}_{x}, \mathscr{D}_{x}\right] .
\end{aligned}
$$

Hence $\partial^{k} \mathscr{D}$ (resp. $\left.\partial^{(k)} \mathscr{D}\right)$ is a subsheaf of $\partial^{k+1} \mathscr{D}\left(\right.$ resp. $\left.\partial^{(k+1)} \mathscr{D}\right)$ for $k \geqq 1$ and $\partial^{(k)} \mathscr{D}$ is a subsheaf of $\partial^{k} \mathscr{D}$ for $k \geqq 2$. Let $\partial^{k} D(x)$ and $\partial^{(k)} D(x)(k \geqq 2)$ be the linear subspaces of $T_{x}(M), x \in M$, defined by

$$
\begin{aligned}
\partial^{k} D(x) & =\left\{X_{x} \in T_{x}(M) \mid X \in \partial^{k} \mathscr{D}_{x}\right\}, \\
\partial^{(k)} D(x) & =\left\{X_{x} \in T_{x}(M) \mid X \in \partial^{(k)} \mathscr{D}_{x}\right\} .
\end{aligned}
$$

If $\operatorname{dim} \partial^{k} D(x)\left(\right.$ resp. $\left.\operatorname{dim} \partial^{(k)} D(x)\right)$ is constant on $M$, the assignment $x \mapsto \partial^{k} D(x)$ (resp. $\partial^{(k)} D(x)$ ) defines the differential system $\partial^{k} D$ (resp. $\partial^{(k)} D$ ) on $M$ such that $\partial^{k} \mathscr{D}$ (resp. $\left.\partial^{(k)} \mathscr{D}\right)$ coincides with the sheaf of $\partial^{k} D$ (resp. $\left.\partial^{(k)} D\right)$. In this case we say that $\partial^{k} \mathscr{D}$ (resp. $\left.\partial^{(k)} \mathscr{D}\right)$ defines a differential system. $\partial^{k} D$ is called the $k$-th derived system of $D$ and $\partial^{(k)} D$ is called the $k$-th weak derived system of $D$. The notion of the weak derived systems was introduced by N. Tanaka [19]. 
$D$ is called regular if $\partial^{(k)} \mathscr{D}$ defines a differential system for every $k \geqq 1$ (Definition 1.1 [19]). If $D$ is regular, there exists a unique integer $\mu>0$ such that

$$
\cdots=\partial^{(\mu)} D=\partial^{(\mu-1)} D \subseteq \partial^{(\mu-2)} D \subseteq \cdots \subseteq \partial^{(1)} D \subseteq D .
$$

$\mu$ being as above, $D$ is called a regular differential system of $\mu$-th kind. One should note that if $D$ is of $\mu$-th kind, then $\partial^{(\mu-1)} \mathscr{D}_{x}$ is the subalgebra of $\mathscr{T}_{x}$ generated by $\mathscr{D}_{x}$ at each $x \in M$ (cf. Proposition 1.1 [19]).

1.3. Graded algebras associated with differential systems. First we will recall the notion of the graded algebra $m(x)$ of a regular differential system $(M, D)$ at $x \in M$, following [19]. Let $D$ be a regular differential system of $\mu$-th kind on $M$ such that $T(M)=\partial^{(\mu-1)} D$. We set $D^{-k-1}=\partial^{(k)} D$ for $k \geqq 1$ and $D^{-1}=D$. Let $x$ be any point of $M$. Then $T(M)=D^{p}$ for $p \leqq-\mu$ and $\mathscr{T}_{x}=\mathscr{D}_{x}^{-\mu}$ is generated, as a Lie algebra, by $\mathscr{D}_{x}$. From $\mathscr{D}_{x}^{p}=\mathscr{D}_{x}^{p+1}+\left[\mathscr{D}_{x}^{p+1}\right.$, $\left.\mathscr{D}_{x}^{-1}\right]$, it follows that the family $\left\{\mathscr{D}_{x}^{p}\right\}_{p<0}$ defines a filtration of $\mathscr{T}_{x}$ and that $\mathscr{T}_{x}$ becomes a (infinite dimensional) filtered Lie algebra with respect to this filtration, i.e.,

$$
\left[\mathscr{D}_{x}^{p}, \mathscr{D}_{x}^{q}\right] \subset \mathscr{D}_{x}^{p+q} \quad \text { for } p, q<0 .
$$

Since $\mathscr{D}_{x}^{p}$ is an $\mathscr{R}_{x}$-submodule of $\mathscr{T}_{x}$ for $p<0$, we can define the (finite dimensional) associated graded Lie algebra $\mathfrak{m}(x)$ of $\mathscr{T}_{x}$ as follows: We set $\mathfrak{g}_{-1}(x)=$ $D^{-1}(x), \mathfrak{g}_{p}(x)=D^{p}(x) / D^{p+1}(x)(p<-1)$ and $\mathfrak{m}(x)=\sum_{p<0} \mathfrak{g}_{p}(x)$ (direct sum). Let $\Pi_{p}$ be the projection of $D_{p}(x)$ onto $\mathfrak{g}_{p}(x)$. Then $\operatorname{dim} \mathfrak{m}(x)=\operatorname{dim} M$ and the product $[X, Y]$ of $X \in \mathfrak{g}_{p}(x)$ and $Y \in \mathfrak{g}_{q}(x)$ is defined by

$$
[X, Y]=\Pi_{p+q}\left([\tilde{X}, \tilde{Y}]_{x}\right) \in \mathfrak{g}_{p+q}(x),
$$

where $\tilde{X}$ and $\tilde{Y}$ are any elements of $\mathscr{D}_{x}^{p}$ and $\mathscr{D}_{x}^{q}$ respectively such that $\Pi_{p}\left(\tilde{X}_{x}\right)$ $=X$ and $\Pi_{q}\left(\tilde{Y}_{x}\right)=Y$. Since $\mathscr{D}_{x}^{p}$ is a free $\mathscr{R}_{x}$-module for $p<0$ and $[f \tilde{X}, g \tilde{Y}]=$ $f \cdot g[\tilde{X}, \tilde{Y}]+f(\tilde{X} g) \tilde{Y}-g(\tilde{Y} f) \tilde{X}$ for $\tilde{X}, \tilde{Y} \in \mathscr{T}_{x}$ and $f, g \in \mathscr{R}_{x}$, it follows immediately that $[X, Y]$ is well-defined for $X \in \mathfrak{g}_{p}(x)$ and $Y \in \mathfrak{g}_{q}(x)$ (cf. Lemma 1.1 [19]). Note that $\mathfrak{g}_{p}(x)=0$ for $p<-\mu, \mathfrak{g}_{-\mu}(x) \neq 0$ and $\left[\mathfrak{g}_{p}(x), \mathfrak{g}_{-1}(x)\right]=\mathfrak{g}_{p-1}(x)$, i.e., $\mathfrak{m}(x)$ is a fundamental graded algebra of $\mu$-th kind (Definition 1.3 [19]). $\mathfrak{m}(x)$ is called the graded algebra of $(M, D)$ at $x \in M$. Furthermore, let $\mathfrak{m}$ be a fundamental graded algebra of $\mu$-th kind. Then $D$ is said to be of type $\mathfrak{m}$ if $\mathfrak{m}(x)$ is isomorphic with $\mathfrak{m}$ at each $x \in M$ (Definition 1.4 [19]). For the notion of standard differential system of type $\mathrm{m}$, we refer to $\S 2$ of [19].

Let $D$ be a regular differential system on $M$ such that $T(M)=\partial^{(\mu-1)} D$. Next we will consider the graded subalgebra of $m(x)$ associated with a submanifold of $M$. Let $N$ be a submanifold of $M$ and c be the inclusion of $N$ into $M$. For each $y \in N$, we set 


$$
C^{p}(y)=\left\{X \in T_{y}(N) \mid \iota_{*}(X) \in D^{p}(\iota(y))\right\} \quad \text { for } p<0 .
$$

If $\operatorname{dim} C^{p}(y)$ is constant on $N$, the assignment $y \mapsto C^{p}(y)$ defines a differential system $C^{p}$ on $N$. In the following we assume that $\operatorname{dim} C^{p}(y)$ is constant on $N$ for every $p<0$. Let $\mathscr{C}^{p}$ be the sheaf of $C^{p}(p<0)$. Let $y$ be any point of $N$. For $X \in \mathscr{C}_{y}^{p}$, let $\bar{X}$ be a representative local vector field of $X$ around $y$. Then $\bar{X}$ can be locally extended to a local section $\bar{X}^{\prime}$ of $D^{p}$ around $\iota(y)$. Let $X^{\prime}$ be the element of $\mathscr{D}_{i(y)}^{p}$ represented by $\bar{X}^{\prime}$. In this case we say that $X^{\prime} \in \mathscr{D}_{\iota(y)}^{p}$ is an extension of $X \in \mathscr{C}_{y}^{p}$. Let $Y^{\prime} \in \mathscr{D}_{b(y)}^{q}$ be an extension of $Y \in \mathscr{C}_{y}^{q}$. Then it is obvious that $\left[X^{\prime}, Y^{\prime}\right]$ is an extension of $[X, Y]$. From $\left[X^{\prime}, Y^{\prime}\right] \in \mathscr{D}_{\iota(y)}^{p+q}$, it follows that $[X, Y] \in \mathscr{C}_{y}^{p+q}$, i.e.,

$$
\left[\mathscr{C}_{y}^{p}, \mathscr{C}_{y}^{q}\right] \subset \mathscr{C}_{y}^{p+q} .
$$

Hence the family $\left\{\mathscr{C}_{y}^{p}\right\}_{p<0}$ defines a filtration of $\mathscr{T}(N)_{y}$ such that $\mathscr{T}(N)_{y}$ is a filtered Lie algebra with respect to this filtration, where $\mathscr{T}(N)$ is the sheaf of germs of local vector fields on $N$. Therefore, similarly as above, we can define the associated graded Lie algebra $\mathfrak{n}(y)=\sum_{p<0} \mathfrak{n}_{p}(y)$ of $\mathscr{T}(N)_{y}$, where $\mathfrak{n}_{-1}(y)=C^{-1}(y)$ and $\mathfrak{n}_{p}(y)=C^{p}(y) / C^{p+1}(y)(p<-1)$. $\mathfrak{n}(y)$ is considered canonically as a graded subalgebra of $m(\iota(y))$ as follows: Let $\hat{\Pi}_{p}$ be the projection of $C^{p}(y)$ onto $\mathfrak{n}_{p}(y)(p<-1)$. Since $\iota_{*}\left(C^{p}(y)\right)=D^{p}(\iota(y)) \cap \iota_{*}\left(T_{y}(N)\right), \iota_{*} ; T_{y}(N)$ $\rightarrow T_{\iota(y)}(M)$ induces an injective linear map $\iota_{*}^{p}$ of $\mathfrak{n}_{p}(y)$ into $\mathfrak{g}_{p}(\iota(y))$ satisfying $\iota_{*}^{p} \cdot \hat{\Pi}_{p}=\left.\Pi_{p} \cdot \iota_{*}\right|_{C^{p}(y)}(p<-1)$. Set $\hat{\iota}_{*}=\iota_{*}^{-\mu} \times \cdots \times \iota_{*}^{-1}$, where $\iota_{*}^{-1}=\left.\iota_{*}\right|_{c^{-1(y)}}$. Then $\hat{\iota}_{*}$ is an injective linear map of $\mathfrak{n}(y)$ into $\mathfrak{m}(\iota(y))$. Let $X \in \mathfrak{n}_{p}(y), Y \in \mathfrak{n}_{q}(y)$. The product $[X, Y]$ of $X$ and $Y$ is defined by

$$
[X, Y]=\hat{\Pi}_{p+q}\left([\tilde{X}, \tilde{Y}]_{y}\right) \in \mathfrak{n}_{p+q}(y),
$$

where $\tilde{X}$ and $\tilde{Y}$ are any elements of $\mathscr{C}_{y}^{p}$ and $\mathscr{C}_{y}^{q}$ respectively such that $\hat{\Pi}_{p}\left(\tilde{X}_{y}\right)$ $=X$ and $\hat{\Pi}_{q}\left(\tilde{Y}_{y}\right)=Y$. Take extensions $\tilde{X}^{\prime} \in \mathscr{D}_{\iota}^{p}$ and $\tilde{Y}^{\prime} \in \mathscr{D}^{q}(y)$ of $\tilde{X}$ and $\tilde{Y}$. Then $\iota_{*}\left(\tilde{X}_{y}\right)=\tilde{X}_{\iota(y)}^{\prime}, \iota_{*}\left(\tilde{Y}_{y}\right)=\tilde{Y}_{\iota(y)}^{\prime}$ and $\iota_{*}\left([\tilde{X}, \tilde{Y}]_{y}\right)=\left[\tilde{X}^{\prime}, \tilde{Y}^{\prime}\right]_{\iota(y)}$. Hence we have $\Pi_{p}\left(\tilde{X}_{\iota(y)}^{\prime}\right)=\iota_{*}^{p}(X), \Pi_{q}\left(\tilde{Y}_{\iota(y)}^{\prime}\right)=\iota_{*}^{q}(Y)$ and $\iota_{*}^{p+q}([X, Y])=\Pi_{p+q}\left(\left[\tilde{X}^{\prime}, \tilde{Y}^{\prime}\right]_{\iota(y)}\right)$. By the definition of the bracket operation of $\mathrm{m}(\iota(y))$, we have $\left[\iota_{*}^{p}(X), \iota_{*}^{q}(Y)\right]=\Pi_{p+q}$ $\left(\left[\tilde{X}^{\prime}, \tilde{Y}^{\prime}\right]_{\iota(y)}\right)$. Hence $\iota_{*}^{p+q}([X, Y])=\left[\iota_{*}^{p}(X), \iota_{*}^{q}(Y)\right]$, i.e., $\hat{\iota}_{*}$ is a graded Lie algebra isomorphism of $\mathfrak{n}(y)$ into $\mathfrak{m}(\iota(y))$. $\mathfrak{n}(y)$, identified with $\hat{\iota}_{*}(\mathfrak{n}(y))$, is called the graded subalgebra of $\mathrm{m}(\iota(y))$ associated with $N$ at $y$.

Now we will consider another type of graded algebras associated with differential systems. Let $D^{1}, \cdots, D^{\nu}$ be a family of differential systems on $M$ such that $\partial \mathscr{D}^{i+1}$ is a subsheaf of $\mathscr{D}^{i}$ for $i=1, \cdots, \nu-1$, where $\mathscr{D}^{i}$ is the sheaf of $D^{i}$. Set $\mathscr{S}^{-i}=\mathscr{D}^{\nu+1-i}$ for $i=1, \cdots, \nu$ and let $x$ be any point of $M$. Then we have $\mathscr{S}_{x}^{p-1} \supset \partial \mathscr{S}_{x}^{p}=\mathscr{S}_{x}^{p}+\left[\mathscr{S}_{x}^{p}, \mathscr{S}_{x}^{p}\right]$ for $p=-1, \cdots,-\nu+1$. Hence it follows that

$$
\left[\mathscr{S}_{x}^{p}, \mathscr{S}_{x}^{q}\right] \subset \mathscr{S}_{x}^{m(p, q)-1} \subset \mathscr{S}_{x}^{p+q} \quad \text { for } p, q<0
$$


where $m(p, q)$ denotes the smaller element of $\{p, q\}$ and we set $\mathscr{S}_{x}^{p}=\mathscr{T}_{x}$ for $p<-\nu$. Therefore, at any rate, the family $\left\{\mathscr{S}_{x}^{p}\right\}_{p<0}$ defines a filtration of $\mathscr{T}_{x}$ such that $\mathscr{T}_{x}$ is a filtered Lie algebra with respect to this filtration. $\mathscr{S}_{x}^{p}=$ $\mathscr{D}_{x}^{p+\nu+1}$ is a free $\mathscr{R}_{x}$-submodule of $\mathscr{T}_{x}$ for each $p<0$. Hence, similarly as above, we can define the associated graded Lie algebra $\mathfrak{S}(x)$ of $\mathscr{T}_{x}$ as follows: We set $\mathfrak{g}_{-1}(x)=D^{k}(x), \mathfrak{g}_{p}(x)=D^{p+k+1}(x) / D^{p+k+2}(x)(p<-1)$ and $\mathfrak{g}(x)=\sum_{p<0} \mathfrak{g}_{p}(x)$ (direct sum). Let $\Pi_{p}$ be the projection of $D^{p+k+1}(x)$ onto $\mathfrak{S}_{p}(x)$. Then $\operatorname{dim} \mathfrak{s}(x)$ $=\operatorname{dim} M$ and the product $[X, Y]$ of $X \in \mathfrak{I}_{p}(x)$ and $Y \in \mathfrak{S}_{q}(x)$ is defined by

$$
[X, Y]=\Pi_{p+q}\left([\tilde{X}, \tilde{Y}]_{x}\right) \in \mathfrak{B}_{p+q}(x),
$$

where $\tilde{X}$ and $\tilde{Y}$ are any elements of $\mathscr{S}_{x}^{p}$ and $\mathscr{S}_{x}^{q}$ respectively such that $\pi_{p}\left(\tilde{X}_{x}\right)$ $=X$ and $\pi_{q}\left(\tilde{Y}_{x}\right)=Y$. Then one should note that

$$
\left[\mathfrak{S}_{p}(x), \mathfrak{I}_{q}(x)\right]=0 \quad \text { if } p, q<-1 .
$$

$\mathfrak{S}(x)$ is called the graded algebra of $\left(M: D^{1}, \cdots, D^{\nu}\right)$ at $x \in M$. We will consider graded algebras of this type in $\S 5$.

1.4. Cauchy-Cartan characteristic systems. Let $D$ be a differential system on $M$. First we define the Cauchy-Cartan characteristic subspace $C h(D)(x)$ of $D$ at $x \in M$ by setting

$$
\left.C h(D)(x)=\{X \in D(x) \mid X\lrcorner(\mathrm{d} \omega)_{x} \equiv 0\left(\bmod D^{\perp}(x)\right) \text { for any } \omega \in \mathscr{D} \frac{\perp}{x}\right\},
$$

where - is the interior multiplication (cf. [7, p. 52] or [18, p. 135]). One should note that, when $D$ is a regular differential system, $C h(D)(x)$ is also defined, as a subspace of $g_{-1}(x)=D(x)$, by

$$
C h(D)(x)=\left\{X \in \mathfrak{g}_{-1}(x) \mid\left[X, \mathfrak{g}_{-1}(x)\right]=0\right\} .
$$

Next we will consider the (cotangent) Cauchy-Cartan characteristic sheaf $C h\left(\mathscr{D}^{\perp}\right)$ of $D$. Let $\mathscr{D}^{\perp}$ be the sheaf of $D^{\perp}$. For each open subset $U$ of $M, \mathscr{D}^{\perp}(U)$ is an $\mathscr{R}(U)$-submodule of $\mathscr{T}^{*}(U)$. We define linear subspaces $\mathscr{L}(U)$ and $\check{\mathscr{C}}^{*}(U)$ of $\mathscr{T}^{*}(U)$ by setting

$$
\begin{aligned}
& \mathscr{L}(U)=\left\{L_{X} \omega \mid X \in \mathscr{D}(U), \quad \text { and } \quad \omega \in \mathscr{D}^{\perp}(U)\right\}, \\
& \check{\mathscr{C}}^{*}(U)=\mathscr{D}^{\perp}(U)+\mathscr{L}(U),
\end{aligned}
$$

where $L_{X}$ denotes the Lie differentiation with respect to $X$. By $\left.L_{X} \omega=X\right\lrcorner \mathrm{d} \omega$ for $X \in \mathscr{D}(U)$ and $\omega \in \mathscr{D}^{\perp}(U)$, it is easy to see that $\check{\mathscr{C}}^{*}(U)$ is an $\mathscr{R}(U)$-submodule of $\mathscr{T}^{*}(U)$. The (cotangent) Cauchy-Cartan characteristic sheaf $C h\left(\mathscr{D}^{\perp}\right)$ of $D$ is, by definition, the sheaf generated by the presheaf: $U \mapsto \check{\mathscr{C}}^{*}(U)$. $\mathrm{Ch}\left(\mathscr{D}^{\perp}\right)$ is a subsheaf of $\mathscr{R}$-modules of $\mathscr{T}^{*}$. We can define the linear subspace $C h\left(D^{\perp}\right)(x)$ of $T_{x}^{*}(M)$ by 


$$
C h\left(D^{\perp}\right)(x)=\left\{\omega_{x} \in T_{x}^{*}(M) \mid \omega \in C h\left(\mathscr{D}^{\perp}\right)_{x}\right\}
$$

Then we have

\section{Lemma 1.2. $\quad C h\left(D^{\perp}\right)(x)=(C h(D)(x))^{\perp}$}

Proof. Let $X_{1}, \cdots, X_{r}$ (resp. $\omega_{1}, \cdots, \omega_{m-r}$ ) be a free basis of $\mathscr{R}_{x}$-module $\mathscr{D}_{x}$ (resp. $\left.\mathscr{D} \frac{1}{x}\right)$. For $X=\sum f_{i} X_{i} \in \mathscr{D}_{x}$ and $\omega=\sum g_{j} \omega_{j} \in \mathscr{D}_{\frac{1}{x}}$, we have

$$
L_{x} \omega=X \smile \mathrm{d} \omega=\sum f_{i} \cdot \mathrm{d} g_{j}\left(X_{i}\right) \omega_{j}+\sum f_{i} g_{j}\left(X_{i} \downarrow \mathrm{d} \omega_{j}\right) .
$$

Hence $C h\left(\mathscr{D}^{\perp}\right)_{x}$ is spanned, as an $\mathscr{R}_{x}$-module, by $\omega_{j}$ and $\left.X_{i}\right\lrcorner \mathrm{d} \omega_{j}(1 \leqq i \leqq r$, $1 \leqq j \leqq m-r)$. Let $X$ be any element of $C h(D)(x)$. Then we have $\left(\omega_{j}\right)_{x}(X)=0$, $\left.\left.\left(X_{i}\right\lrcorner \mathrm{d} \omega_{j}\right)_{x}(X)=X\right\lrcorner\left(\mathrm{d} \omega_{j}\right)_{x}\left(X_{i}\right)=0$. Therefore $C h\left(D^{\perp}\right)(x) \subset(C h(D)(x))^{\perp}$.

Conversely, let $Y$ be any element of $\left(\mathrm{Ch}\left(D^{\perp}\right)(x)\right)^{\perp}$, i.e., $\left(\omega_{j}\right)_{x}(Y)=$ $\left.\left(X_{i}\right\lrcorner \mathrm{d} \omega_{j}\right)_{x}(Y)=0$ for $i=1, \cdots, r$ and $j=1, \cdots, m-r$. From $\left(\omega_{j}\right)_{x}(Y)=0$ for $j=1, \cdots, m-r$, we have $Y \in D(x)$. And from $\left.\left.\left(X_{i}\right\lrcorner \mathrm{d} \omega_{j}\right)_{x}(Y)=Y\right\lrcorner\left(\mathrm{d} \omega_{j}\right)_{x}\left(X_{i}\right)=0$, we get $Y\lrcorner\left(\mathrm{d} \omega_{j}\right)_{x} \equiv 0\left(\bmod D^{\perp}(x)\right)$ for $j=1, \cdots, m-r$. Therefore $Y \in C h(D)(x)$.

q.e.d.

If $\operatorname{dim} C h(D)(x)$ is constant on $M$, then it is easily seen that the assignment $x \mapsto C h(D)(x)$ defines the differential system $C h(D)$ on $M$ such that $C h\left(\mathscr{D}^{\perp}\right)$ coincides with the sheaf of the annihilator bundle $(C h(D))^{\perp}$ of $C h(D)$. $C h(D)$ is called the Cauchy-Cartan characteristic system of $D$.

Let $C h(\mathscr{D})$ be the subsheaf of $\mathscr{R}$-modules of $\mathscr{D}$ generated by the presheaf; $U \mapsto \check{\mathscr{C}}(U)=\{X \in \mathscr{D}(U) \mid[X, \mathscr{D}(U)] \subset \mathscr{D}(U)\}$. And we set

$$
\check{C}(x)=\left\{X_{x} \in T_{x}(M) \mid X \in C h(\mathscr{D})_{x}\right\} .
$$

In general we have $\check{C}(x) \subset C h(D)(x)$. One should note that, if $\operatorname{dim} C h(D)(x)$ is constant on $M$, then $\check{C}(x)$ coincides with $C h(D)(x)$ at each $x \in M$ and that, in this case, $C h(\mathscr{D})$ coincides with the sheaf of $C h(D)$. Furthermore it is easy to see that $C h(\mathscr{D})$ is a sheaf of Lie algebras. Hence, as is well known, $C h(D)$ is completely integrable.

LEMmA 1.3. Let $C$ and $D$ be differential systems on $M$ such that the derived sheaf $\partial \mathscr{D}$ of $D$ is a subsheaf of the sheaf $\mathscr{C}$ of $C$. Let $x$ be a point of $M$. Then $C h(D)(x) \subset C h(C)(x)$ if $C(x)=\partial D(x)$. Hence, in particular, if $\operatorname{dim} \partial D(x)$ is constant on $M$,

$$
C h(D)(x) \subset C h(\partial D)(x) \quad \text { at each } x \in M,
$$

where $\partial D$ is the first derived system of $D$.

Proof. Let $\omega_{1}, \cdots, \omega_{r+s}$ be 1 -forms on a neighborhood $U$ of $x$ such that $D$ is defined on $U$ by $\omega_{1}, \cdots, \omega_{r+s}$ and that $C$ is defined on $U$ by $\omega_{1}, \cdots, \omega_{r}$, 
where $r=\operatorname{codim} C$ and $r+s=\operatorname{codim} D . \quad$ From $\partial \mathscr{D} \subset \mathscr{C}$ and Lemma 1.1, we get

$$
\mathrm{d} \omega_{\alpha} \equiv 0\left(\bmod \omega_{1}, \cdots, \omega_{r+s}\right) \quad \text { for } \quad \alpha=1, \cdots, r \text { on } U \text {. }
$$

Hence there exist 1 -forms $\pi_{j}^{\alpha}(1 \leqq \alpha \leqq r, 1 \leqq j \leqq s)$ on $U$ such that

$$
\mathrm{d} \omega_{\alpha} \equiv \sum_{j=1}^{s} \pi_{j}^{\alpha} \wedge \omega_{r+j}\left(\bmod \omega_{1}, \cdots, \omega_{r}\right) \text { for } \alpha=1, \cdots, r \text { on } U .
$$

Differentiating both sides, we get

$$
\sum_{j=1}^{s} \pi_{j}^{\alpha} \wedge \mathrm{d} \omega_{r+j} \equiv 0\left(\bmod \omega, \cdots, \omega_{r+s}\right) \text { for } \alpha=1, \cdots, r \text { on } U .
$$

Let $X$ be any element of $C h(D)(x)$. Then $\omega_{i}(X)=0$ and $\left.X\right\lrcorner\left(\mathrm{d} \omega_{i}\right)_{x}=0$ $\left(\bmod D^{\perp}(x)\right)(1 \leqq i \leqq r+s)$. Hence we have

$$
\sum_{j=1}^{s} \pi_{j}^{\alpha}(X)\left(\mathrm{d} \omega_{r+j}\right)_{x} \equiv 0\left(\bmod D^{\perp}(x)\right) \text { for } \quad \alpha=1, \cdots, r .
$$

On the other hand, from Lemma 1.1, it follows that $C(x)=\partial D(x)$ if and only if $\left(\mathrm{d} \omega_{r+1}\right)_{x}, \cdots,\left(\mathrm{d} \omega_{r+s}\right)_{x}$ are linearly independent modulo $D^{\perp}(x)$. Hence we get $\pi_{j}^{\alpha}(X)=0$ for $1 \leqq \alpha \leqq r, 1 \leqq j \leqq s$. Therefore we obtain

$$
X\lrcorner\left(\mathrm{d} \omega_{\alpha}\right)_{x} \equiv 0\left(\bmod C^{\perp}(x)\right) \quad \text { for } \quad \alpha=1, \cdots, r,
$$

which implies $X \in C h(C)(x)$.

q.e.d.

REMARK 1.4 (Covariant systems). Let $M$ and $\hat{M}$ be manifolds of dimension $m$. Let $D$ and $\hat{D}$ be differential systems on $M$ and $\hat{M}$ respectively. Assume that there exists an isomorphism $\phi$ of $(M, D)$ onto $(\hat{M}, \hat{D})$. Then, since $k$-th derived systems, $k$-th weak derived systems and Cauchy-Cartan characteristic systems are canonically constructed, it follows that $\phi_{*}\left(\partial^{k} D\right)=$ $\partial^{k} \hat{D}, \quad \phi_{*}\left(\partial^{(k)} D\right)=\partial^{(k)} \hat{D}(k \geqq 1)$ and $\phi_{*}(C h(D))=C h(\hat{D})$. In other words $\partial^{k} D$, $\partial^{(k)} D(k \geqq 1)$ and $C h(D)$ are covariant systems of $D$ in the sense of E. Cartan [5] or [6]. We will consider another type of covariant systems in $\S 7$.

1.5. Canonical systems on Grassmann bundles. Let $M$ be a manifold of dimension $m+n$. We consider the Grassmann bundle $J(M, n)$ over $M$ consisting of $n$-dimensional contact elements of $M$, i.e.,

$$
J(M, n)=\bigcup_{x \in M} G r\left(T_{x}(M), n\right),
$$

where $\operatorname{Gr}\left(T_{x}(M), n\right)$ denotes the Grassmann manifold of $n$-dimensional subspaces of $T_{x}(M)$ (cf. [14, p. 44]). Let $\Pi$ be the bundle projection of $J(M, n)$ onto $M$. Let $z \in J(M, n)$. Then $\Pi_{*}: T_{z}(J(M, n)) \rightarrow T_{x}(M)$ is onto and $z$ is an $n$-dimensional subspace of $T_{x}(M)$, where $x=\Pi(z)$. The canonical (differential) 
system $C^{*}$ on $J(M, n)$ is, by definition, the differential system of codimension $m$ on $J(M, n)$ given by

$$
C^{*}(z)=\left\{X \in T_{z}(J(M, n)) \mid \Pi_{*}(X) \in z\right\}\left(=\Pi_{*}^{-1}(z)\right) \text { for } z \in J(M, n) .
$$

We will give a canonical coordinate system on $J(M, n)$. Let $z_{0}$ be any point of $J(M, n)$. Let $\left(z_{1}^{\prime}, \cdots, z_{m}^{\prime}, x_{1}^{\prime}, \cdots, x_{n}^{\prime}\right)$ be a coodinate system on a neighborhood $U^{\prime}$ of $x_{0}=\Pi\left(z_{0}\right)$ such that $\mathrm{d} x_{1}^{\prime}, \cdots, \mathrm{d} x_{n}^{\prime}$ are linearly independent on $z_{0}$. We set $U=\left\{z \in \Pi^{-1}\left(U^{\prime}\right) \mid \mathrm{d} x_{1}^{\prime}, \cdots, \mathrm{d} x_{n}^{\prime}\right.$ are linearly independent on $\left.z\right\}$. Then $U$ is a neighborhood of $z_{0}$ in $J(M, n)$. There exist unique functions $p_{i}^{\alpha}$ on $U$ such that $C^{*}$ is defined on $U$ by the following 1 -forms;

$$
\pi^{\alpha}=\mathrm{d} z^{\alpha}-\sum_{i=1}^{n} p_{i}^{\alpha} \mathrm{d} x_{i}(\alpha=1, \cdots, m),
$$

where $z^{\alpha}=z_{\alpha}^{\prime} \cdot \Pi$ and $x_{i}=x_{i}^{\prime} \cdot \Pi$. The system of functions $z^{\alpha}, x_{i}$ and $p_{i}^{\alpha}(\alpha=1$, $\cdots, m, i=1, \cdots, n$ ) form a coordinate system on $U$, which is called the canonical coordinate system of $J(M, n)$ subordinate to $\left(z_{\alpha}^{\prime}, x_{i}^{\prime}\right)$ (cf. Lemma 2.2). By using canonical coordinate systems, we see easily that the Cauchy-Cartan characteristic system $C h\left(C^{*}\right)$ of $C^{*}$ is trivial, i.e., $C h\left(C^{*}\right)(z)=\{0\}$ for any $z \in J(M, n)$. For a more detailed study of the canonical system $C^{*}$ on $J(M, n)$, we refer to [21].

Let $R$ be a manifold. Let $p$ be a differentiable map of $R$ into $M$ of constant rank, i.e., rank $\left(p_{*}\right)_{y}$ is constant on $R$. Then it is easy to see that the assignment $y \mapsto \operatorname{Ker}\left(p_{*}\right)_{y}$ defines a completely integrable differential system $\operatorname{Ker} p_{*}$ on $R$.

We need the following lemma in $\S 5$, which is a slight generalization of the one given by N. Tanaka [20]. His result was communicated to us in his lecture on [20] at Hokkaido University in the summer of 1980. Though the following proof is essentially the same as in [20], we will give it for the sake of completeness.

Lemma 1.5 (Realization Lemma) (cf. [20]). Let $R$ and $M$ be manifolds. Assume that the quadruple $(R, C, p, M)$ satisfies the following conditions:

(i) $p$ is a map of $R$ into $M$ of constant rank.

(ii) $C$ is a differential system on $R$ such that $F=\operatorname{Ker} p_{*}$ is a subbundle of $C$ of codimension $n$.

Then there exists a unique map $\psi$ of $R$ into $J(M, n)$ satisfying $p=\Pi \cdot \psi$ and $C=\psi_{*}^{-1}\left(C^{*}\right)$. Furthermore, let $y$ be any point of $R$. Then $\psi$ is in fact defined by

$$
\psi(y)=p_{*}(C(y)) \text { as a point of } \operatorname{Gr}\left(T_{p(y)}(M)\right)
$$

and satisfies 


$$
\operatorname{Ker}\left(\psi_{*}\right)_{y}=F(y) \cap C h(C)(y) .
$$

Proof. By $C=\psi_{*}^{-1}(C)$, we have $C(y)=\psi_{*}^{-1}\left(C^{*}(\psi(y))\right)$, i.e.,

$$
\psi^{*}(C(y))=C^{*}(\psi(y)) \cap \psi_{*}\left(T_{y}(R)\right) .
$$

Furthermore, by $p=\Pi \cdot \psi, C \supset \operatorname{Ker} p_{*}$ and $C^{*} \supset \operatorname{Ker} \Pi_{*}$, this is equivalent to

$$
p_{*}(C(y))=\Pi_{*}\left(C^{*}(\psi(y))\right) \cap p_{*}\left(T_{y}(R)\right) .
$$

On the other hand, by the definition of the canonical system $C^{*}$, we have $\Pi_{*}\left(C^{*}(\psi(y))\right)=\psi(y)$. And, by (ii), $p_{*}(C(y))$ is an $n$-dimensional subspace of $T_{p(y)}(M)$. Therefore we get $\psi(y)=p_{*}(C(y))$. This shows the existence and the uniqueness of $\psi$.

Let $\pi_{1}, \cdots, \pi_{m}$ be 1 -forms defined on a neighborhood $U$ of $\psi(y)$ such that $C^{*}$ is defined on $U$ by $\pi_{1}, \cdots, \pi_{m}$. Then, by $C=\psi_{*}^{-1}\left(C^{*}\right), C$ is defined on $\hat{U}=$ $\psi^{-1}(U)$ by $\psi^{*} \pi_{1}, \cdots, \psi^{*} \pi_{m}$. Let $X$ be any element of $\operatorname{Ker}\left(\psi_{*}\right)_{y}$. Then, by (ii), we have $X \in F(y) \cap C(y)$. Furthermore, from $X-\psi^{*} \mathrm{~d} \pi_{\alpha}=0(\alpha=1, \cdots, m)$, we get $X \in C h(C)(y)$. Hence $X \in F(y) \cap C h(C)(y)$.

Conversely, let $X$ be any element of $F(y) \cap C h(C)(y)$. By (ii), we have $\psi_{*}(X) \in \operatorname{Ker} \Pi_{*}(\psi(y)) \subset C^{*}(\psi(y))$. And from $\left.X\right\lrcorner \psi^{*} \mathrm{~d} \pi_{\alpha} \equiv 0\left(\bmod C^{\perp}(y)\right)$ for $1 \leqq \alpha \leqq m$, we see that $\psi_{*}(X) \_\mathrm{d} \pi_{\alpha}$ vanishes on $\psi_{*}(C(y))$. On the other hand we have $C^{*}(\psi(y))=\psi_{*}(C(y))+\left(\operatorname{Ker} \Pi_{*}\right)(\psi(y))$. Since $\psi_{*}(X) \in \operatorname{Ker} \Pi_{*}(\psi(y))$ and $\mathrm{d} \pi_{\alpha}$ vanishes on $\operatorname{Ker} \Pi_{*}$, it follows that $\left.\psi_{*}(X)\right\lrcorner \mathrm{d} \pi_{\alpha}$ vanishes on $C^{*}(\psi(y))$. Hence $\psi_{*}(X) \in C h\left(C^{*}\right)(\psi(y))$. Therefore, from $C h\left(C^{*}\right)=\{0\}$, we get $\psi_{*}(X)=0$. q.e.d.

\section{§ 2. Lagrange-Grassmann bundle over $\mathbb{C}$ and its higher prolongations.}

2.1. Lagrange-Grassmann bundle $L(\mathfrak{S})$. Let $\mathfrak{}=(K, C)$ be a contact manifold of dimension $2 n+1$, i.e., $K$ is a manifold of dimension $2 n+1, C$ is a differential system on $K$ of codimension 1 such that $C$ is defined locally by a 1-form $\pi$ satisfying $\pi \wedge(\mathrm{d} \pi)^{n} \neq 0$ at each point. For each point $u$ of $K,(C(u)$, $\left.(\mathrm{d} \pi)_{u}^{\prime}\right)$ is a symplectic vector space of dimension $2 n$, where $(\mathrm{d} \pi)^{\prime}$ is the restriction to $C$ of $\mathrm{d} \pi$.

We first consider the Lagrange-Grassmann bundle $L(\mathfrak{c})$ over $\sqrt{5}$. More precisely, for each $u \in K$, let $L_{u}$ be the set of all $n$-dimensional integral elements of $\widetilde{C}$ at $u$, i.e., $L_{u}$ is the set of all Lagrangian subspaces of $\left(C(u),(\mathrm{d} \pi)_{u}^{\prime}\right)$. Then $L(ङ)$ is the set of all $n$-dimensional integral elements of $\Subset$ :

$$
L(\mathfrak{5})=\bigcup_{u \in K} L_{u} \text {. }
$$

Let $J(K, n)$ be the Grassmann bundle over $K$ of all $n$-dimensional contact 
elements of $K$. Then $L(\Im)$ is a regular submanifold of $J(K, n)$ of dimension $\frac{1}{2} n(n+1)+2 n+1$ (cf. Lemma 2.2). Let $\Lambda(n)$ be the Lagrange-Grassmann manifold of all Lagrangian subspaces in the symplectic vector space of dimension $2 n$ (cf. [2]). Clearly $L($ (ङ) is a fibre bundle over $K$ with standard fibre $\Lambda(n)$. In particular $L_{u}$ is connected for each $u \in K$. Let $\Pi$ be the bundle projection of $L(\mathfrak{S})$ onto $K$. For $v \in L(\Im), \Pi_{*}: T_{v}(L(\Im)) \rightarrow T_{u}(K)$ is onto and $v$ is an $n$-dimensional subspace of $T_{u}(K)$, where $u=\Pi(v)$. The canonical system on $J(K, n)$ induces a differential system on $L(\Im)$.

Definition 2.1. The canonical (differential) system $E$ on $L(\mathfrak{s})$ is the differential system of codimension $n+1$ defined by

$$
E(v)=\left\{X \in T_{v}(L(5)) \mid \Pi_{*}(X) \in v\right\}\left(=\Pi_{*}^{-1}(v)\right) \quad \text { for } v \in L(5) .
$$

We will give a canonical coordinate system on $L(\mathbb{S})$ by the aid of a canonical coordinate system of $\mathfrak{5}$.

LEMma 2.2. For each $v_{0} \in L(\mathbb{S})$, there exists a canonical coordinate system $(x, z, p)=\left(x_{1}^{\prime}, \cdots, x_{n}^{\prime}, z^{\prime}, p_{1}^{\prime}, \cdots, p_{n}^{\prime}\right)$ of S on a neighborhood $U^{\prime}$ of $u_{0}=\Pi\left(v_{0}\right)$ such that $\mathrm{d} x_{1}^{\prime}, \cdots, \mathrm{d} x_{n}^{\prime}$ are linearly independent on $v_{0}$. Set $U=\left\{v \in \Pi^{-1}\left(U^{\prime}\right) \mid \mathrm{d} x_{1}^{\prime}, \cdots\right.$, $\mathrm{d} x_{n}^{\prime}$ are linearly independent on $\left.v\right\}$. Then there exist unique functions $p_{i j}(i, j$ $=1, \cdots, n)$ on $U$ such that

(i) $p_{i j}=p_{j i}$,

(ii) the system of functions $x_{i}, z, p_{i}$ and $p_{i j}(1 \leqq i \leqq j \leqq n)$ is a coordinate system of $L(\mathcal{S})$ on a neighborhood $U$ of $v_{0}$, where $x_{i}=x_{i}^{\prime} \cdot \Pi, z=z^{\prime} \cdot \Pi$ and $p_{i}=$ $p_{i}^{\prime} \cdot \Pi(i=1, \cdots, n)$,

(iii) $E$ is defined on $U$ by the following 1-forms;

$$
\begin{aligned}
\Pi I^{*} \hat{\pi} & =\mathrm{d} z-\sum_{i=1}^{n} p_{i} \mathrm{~d} x_{i}, \\
\hat{\pi}_{i} & =\mathrm{d} p_{i}-\sum_{j=1}^{n} p_{i j} \mathrm{~d} x_{j}(i=1, \cdots, n),
\end{aligned}
$$

where $\hat{\pi}=\mathrm{d} z^{\prime}-\sum_{i=1}^{n} p_{1}^{\prime} \mathrm{d} x_{i}^{\prime}$ is the contact form of $(x, z, p)$.

The coordinate system of $L(5)$ given in Lemma 2.2 is called a canonical coordinate system subordinate to $(x, z, p)$.

Proof. By the Darboux's theorem, we have a canonical coordinate system $(x, z, p)$ on a neighborhood $U^{\prime}$ of $u_{0} \in K$, i.e., $(x, z, p)$ is a coordinate system on $U^{\prime}$ such that $C$ is defined on $U^{\prime}$ by the contact form $\hat{\pi}=\mathrm{d} z^{\prime}-\sum_{i=1}^{n}$ $p_{i}^{\prime} \mathrm{d} x_{i}^{\prime}$. Here, since $v_{0}$ is a Lagrangian subspace of $C\left(u_{0}\right)$, we may assume, by a suitable rearrangement of indices, that there exists an integer $s(1 \leqq s \leqq n)$ 
such that $\left(\mathrm{d} x_{1}^{\prime}\right)_{u_{0}}, \cdots,\left(\mathrm{d} x_{s}^{\prime}\right)_{u_{0}},\left(\mathrm{~d} p_{s+1}^{\prime}\right)_{u_{0}}, \cdots,\left(\mathrm{d} p_{n}^{\prime}\right)_{u_{0}}$ are linearly independent on $v_{0}$ (cf. Lemma 2.2 [16]). Then, by considering the elementary contact transformation given by

$$
\begin{aligned}
\tilde{z} & =z^{\prime}-\sum_{i=s+1}^{n} x_{i}^{\prime} \cdot p_{i}^{\prime}, \tilde{x}_{i}=x_{i}^{\prime}, \tilde{p}_{i}=p_{i}^{\prime}(i=1, \cdots, s), \\
\tilde{x}_{j} & =p_{j}^{\prime}, \tilde{p}_{j}=-x_{j}^{\prime}(j=s+1, \cdots, n),
\end{aligned}
$$

we may assume that $\left(\mathrm{d} x_{1}^{\prime}\right)_{u_{0}}, \cdots,\left(\mathrm{d} x_{n}^{\prime}\right)_{u_{0}}$ are linearly independent on $v_{0}$ (cf. Theorem 4 [4]).

Clearly $U$ is a neighborhood of $v_{0}$ in $L(5)$. We have functions $p_{i j}(i, j=$ $1, \cdots, n)$ on $U$ such that the annihilator subspace of $v$ in $T_{u}^{*}(K)$ is generated by $\hat{\pi}_{u}$ and $\alpha_{i}(v)=\left(\mathrm{d} p_{i}^{\prime}\right)_{u}-\sum_{j=1}^{n} p_{i j}(v)\left(\mathrm{d} x_{j}^{\prime}\right)_{u}(i=1, \cdots, n)$, where $u=\Pi(v)$. The system of functions $x_{i}^{\prime} \cdot \Pi, z^{\prime} \cdot \Pi, p_{i}^{\prime} \cdot \Pi$ and $p_{i j}(i, j=1, \cdots, n)$ is a canonical coordinate system of $J(K, n)$. Since $v$ is an integral element of $(K, C), \mathrm{d} \hat{\pi}_{u}$ vanishes on $v$, i.e., $\mathrm{d} \hat{\pi}_{u}=\sum_{j=1}^{n}\left(\mathrm{~d} x_{i}^{\prime}\right)_{u} \wedge\left(\mathrm{d} p_{i}^{\prime}\right)_{u} \equiv 0\left(\bmod \hat{\pi}_{u}, \alpha_{1}(v), \cdots, \alpha_{n}(v)\right)$. From this, we get $p_{i j}(v)=p_{j i}(v)$ for $i, j=1, \cdots, n$. Hence $L(\mathfrak{S})$ is a regular submanifold of $J(K, n)$ of dimension $\frac{1}{2} n(n+1)+2 n+1$.

It is obvious that the system of functions $x_{i}, z, p_{i}$ and $p_{i j}(1 \leqq i \leqq j \leqq n)$ is a coordinate system of $L\left(\right.$ (5) on $U$ and (iii) holds, where $x_{i}=x_{i}^{\prime} \cdot \Pi, z=z^{\prime} \cdot \Pi$ and $p_{i}=p_{i}^{\prime} \cdot \Pi(i=1, \cdots, n)$.

q.e.d.

One should observe that a contact manifold c can be considered, by virtue of the Darboux's theorem, as an abstraction of the space of 1-jets of one unknown function. In this context, Lemma 2.2 shows that $(L(\mathcal{(}), E)$ corresponds to the space of 2-jets. These being remarked, henceforth, we use the following notations;

$$
J^{1}(\Im)=K, C^{1}=C, J^{2}(\Im)=L(\Im), \rho_{1}^{2}=I I \quad \text { and } \quad Q^{2}=\operatorname{Ker}\left(\rho_{1}^{2}\right)_{*} \cdot
$$

2.2. Higher prolongations $\left(J^{k}(\mathfrak{S}), C^{k}\right)$. By using the above notation, we define the $k$-th order prolongation $\left(J^{k}(\mathfrak{S}), C^{k}\right)$ of $\mathfrak{S}(k \geqq 3)$ inductively as follows.

(1) The bundle $J^{k}$ (ङ) of $k$-th order: For each $u \in J^{k-1}\left(\right.$ (5), let $J_{u}^{k}$ be the set of all $n$-dimensional integral elements $v$ of $\left(J^{k-1}(\mathfrak{S}), C^{k-1}\right)$ at $u$ such that $v \cap Q^{k-1}(u)=\{0\}$, where $Q^{k-1}=\operatorname{Ker}\left(\rho_{k-2}^{k-1}\right)_{*}$. Then $J^{k}(5)$ is defined by

$$
J^{k}(\nwarrow)=\bigcup_{u \in J^{k-1}(\Subset)} J_{u}^{k}(k \geqq 3) .
$$

Let $J\left(J^{k-1}(\Im), n\right)$ be the Grassmann bundle over $J^{k-1}(\Im)$ of all $n$-dimensional contact elements of $J^{k-1}(\mathbb{E})$. Then $J^{k}(\mathbb{E})$ is a regular submanifold of $J\left(J^{k-1}(\mathfrak{S}), n\right) . \quad J^{k}(\mathfrak{S})$ is a fibre bundle over $J^{k-1}(\mathfrak{S})$ with standard fibre $R^{N^{\prime}(k)}$, where $N^{\prime}(k)={ }_{n} H_{h}=\left(\underset{k}{n+k-1}\right.$ ) (cf. Lemma 2.3). Let $\rho_{k-1}^{k}$ be the bundle projection 
of $J^{k}(\mathfrak{S})$ onto $J^{k-1}(\mathfrak{S})$. We set

$$
\rho_{m}^{k}=\rho_{k-1}^{k} \circ \cdots \circ \rho_{m}^{m+1} \text { for } k>m \text { and } \rho_{k}^{k}=i d_{J^{k(\Xi)}} .
$$

(2) The canonical (differential) system $C^{k}$ on $J^{k}(\mathfrak{S})$ : For $v^{k} \in J^{k}(\mathfrak{S})$, $\left(\rho_{k-1}^{k}\right)_{*}: T_{v^{k}}\left(J^{k}(\mathfrak{S})\right) \rightarrow T_{v^{k-1}}\left(J^{k-1}(\mathfrak{S})\right)$ is onto and $v^{k}$ is an $n$-dimensional subspace of $T_{v^{k-1}}\left(J^{k-1}(\mathfrak{C})\right)$, where $v^{k-1}=\rho_{k-1}^{k}\left(v^{k}\right)$. Then $C^{k}$ is defined by

$$
C^{k}\left(v^{k}\right)=\left(\rho_{k-1}^{k}\right)_{*}^{-1}\left(v^{k}\right) \quad \text { for } v^{k} \in J^{k}(\Subset) .
$$

In other words $C^{k}$ is the restriction to $J^{k}(\mathfrak{C})$ of the canonical system on $J\left(J^{k-1}(\mathfrak{C}), n\right)$.

Thus we have completed our inductive definition of $\left(J^{k}(\mathcal{C}), C^{k}\right)$.

Similarly as in Lemma 2.2, we will give a canonical coordinate system of $J^{k}(\mathfrak{S})$ subordinate to a canonical coordinate system on $J^{1}(\mathfrak{5})$. Let $v_{0}^{k}$ be any point of $J^{k}(\mathbb{S})$. Let $\left(x_{1}, \cdots, x_{n}, z, p_{1}, \cdots, p_{n}, p_{11}, \cdots, p_{n n}\right)$ be a canonical coordinate system of $J^{2}(\mathfrak{S})$ on a neighborhood $U$ of $v_{0}^{2}=\rho_{2}^{k}\left(v_{0}^{k}\right)$, which is subordinate to $(x, z, p)$ (Lemma 2.2). Then $U_{m}=\left(\rho_{2}^{m}\right)^{-1}(U)$ is a neighborhood of $v_{0}^{m}=\rho_{m}^{k}\left(v_{0}^{k}\right)(m=3, \cdots, k) . \quad C^{2}$ is defined on $U$ by the following 1-forms;

$$
\begin{aligned}
& \hat{\pi}=\mathrm{d} z-\sum_{i=1}^{n} p_{i} \mathrm{~d} x_{i}, \\
& \hat{\pi}_{i}=\mathrm{d} p_{i}-\sum_{j=1}^{n} p_{i j} \mathrm{~d} x_{j}(i=1, \cdots, n) .
\end{aligned}
$$

And $Q^{2}$ is defined by $\hat{\pi}, \hat{\pi}_{i}$ and $\mathrm{d} x_{i}(i=1, \cdots, n)$.

Let $M_{k}$ be the set of all ordered $k$-tuples of integers $1, \cdots, n$. Hence $p_{I}$ means $p_{i_{1} \cdots i_{k}}$ and $p_{I, j}=p_{i_{1} \cdots i_{k} j}$ for $I=\left(i_{1}, \cdots, i_{k}\right) \in M_{k}$. We denote by $S_{k}$ the set of all $I=\left(i_{1}, \cdots, i_{k}\right) \in M_{k}$ such that $1 \leqq i_{1} \leqq \cdots \leqq i_{k} \leqq n$ and set $\Sigma_{k}=\bigcup_{m=1}^{k} S_{m}$. Note that $\sharp\left(S_{k}\right)={ }_{n} H_{k}$. We set $N(k)=\#\left(\sum_{k}\right)=\sum_{m=1}^{k} H_{m}$. In the following, for a function $f$ (resp. 1-form $\omega$ ) on $U_{m}$, we denote the function $f \cdot \rho_{m}^{k}$ (resp. 1-form $\left.\left(\rho_{m}^{k}\right)^{*} \omega\right)$ on $U_{k}(k>m)$ simply by $f$ (resp. $\omega$ ), when there is no danger of confusion.

Lemma 2.3. Notations being as above, for each $k$ ( $k \geqq 3)$, there exist unique functions $p_{I}\left(I \in M_{k}\right)$ on $U_{k}$ such that

(i ) $p_{I}$ is symmetric with respect to $I$,

(ii) the system of functions $x_{1}, \cdots, x_{n}, z$ and $p_{I}\left(I \in \Sigma_{k}\right)$ is a coordinate system on $U_{k}$,

(iii) $C^{k}$ is defined on $U_{k}$ by the following 1-forms;

$$
\begin{aligned}
& \hat{\pi}=\mathrm{d} z-\sum_{i=1}^{n} p_{i} d x_{i}, \\
& \hat{\pi}_{I}=\mathrm{d} p_{I}-\sum_{j=1}^{n} p_{I, j} d x_{j}\left(I \in \Sigma_{k-1}\right) .
\end{aligned}
$$


Furthermore we can take a canonical coordinate system $(x, z, p)$ of $\sqrt{5}$ on $\left(\rho_{1}^{2}\right)(U)$ such that $x_{i}\left(v_{0}^{1}\right)=z\left(v_{0}^{1}\right)=p_{I}\left(v_{0}^{m}\right)=0$ for $i=1, \cdots, n$ and $I \in M_{m}(m=1$, $\cdots, k)$, where $v_{0}^{m}=\rho_{m}^{k}\left(v_{0}^{k}\right)$.

The coordinate system of $J^{k}(\mathcal{E})$ given in Lemma 2.3 is called the canonical coordinate system subordinate to $(x, z, p)$.

Proof. We proceed by induction on $k$. For $k=2$, we have the canonical coordinate system subordinate to $(x, z, p)$ on $U$ (Lemma 2.2). Assume that, for $m \leqq k-1$, we have defined functions $p_{I}\left(I \in M_{m}\right)$ on $U_{m}$ satisfying (i), (ii) and (iii). Then $Q^{k-1}=\operatorname{Ker}\left(\rho_{k-2}^{k-1}\right)_{*}$ is defined on $U_{k-1}$ by $d x_{1}, \cdots, d x_{n}, \hat{\pi}$ and $\hat{\pi}_{I}\left(I \in \Sigma_{k-2}\right)$. Let $v^{k}$ be any point of $U_{k} . \quad v=v^{k}$ is an $n$-dimensional subspace of $C^{k-1}(u)$ such that $v \cap Q^{k-1}(u)=\{0\}$, where $u=\rho_{k-1}^{k}(v) \in U_{k-1}$, i.e., $C^{k-1}(u)=v \oplus$ $Q^{k-1}(u)$ (direct sum). Hence there exist unique functions $p_{I, j}\left(I \in M_{k-1}, j=1\right.$, $\cdots, n)$ on $U_{k}$ such that $p_{I, j}$ is symmetric with respect to $I$ and that the annihilator subspace of $v$ in $T_{u}^{*}\left(J^{k-1}(\mathfrak{S})\right)$ is generated by $\hat{\pi}_{u},\left(\hat{\pi}_{J}\right)_{u}\left(J \in \Sigma_{k-2}\right)$ and $\alpha_{I}(v)=\left(\mathrm{d} p_{I}\right)_{u}-\sum_{j=1}^{n} p_{I, j}(v)\left(\mathrm{d} x_{j}\right)_{u}\left(I \in S_{k-1}\right)$. The system of functions $x_{i}, z, p_{J}$ and $p_{I, j}\left(i, j=1, \cdots, n, J \in \Sigma_{k-2}, I \in S_{k-1}\right)$ is a canonical coordinate system of $J\left(J^{k-1}(\mathfrak{S}), n\right)$. Since $v$ is an integral element of $\left(J^{k-1}(\mathfrak{S}), C^{k-1}\right)$ at $u, \mathrm{~d} \hat{\pi}_{u}$ and $\left(\mathrm{d} \hat{\pi}_{J}\right)_{u}\left(J \in \Sigma_{k-2}\right)$ vanish on $v$, i.e., $\mathrm{d} \hat{\pi}_{u} \equiv\left(\mathrm{d} \hat{\pi}_{J}\right)_{u} \equiv 0\left(\bmod \hat{\pi}_{u},\left(\hat{\pi}_{J}\right)_{u}, \alpha_{I}(v)\left(J \in \Sigma_{k-2}\right.\right.$, $\left.I \in S_{k-1}\right)$ ). From this we get easily

$$
p_{J, i, j}(v)=p_{J, j, i}(v)\left(i, j=1, \cdots, n, J \in M_{k-2}\right) .
$$

Hence $p_{I}\left(I \in M_{k}\right)$ is symmetric with respect to $I$ and $J^{k}(\mathfrak{S})$ is a regular submanifold of $J\left(J^{k-1}(5), n\right)$ of dimension $N(k)+n+1$.

It is obvious that the system of functions $x_{1}, \cdots, x_{n}, z$ and $p_{I}\left(I \in \Sigma_{k}\right)$ is a coordinate system on $U_{k}$ and (iii) holds.

Furthermore we get easily the last assertion by setting

$$
\begin{aligned}
\tilde{x}_{i} & =x_{i}-x_{i}\left(v_{0}^{1}\right)(i=1, \cdots, n), \\
\tilde{z} & =z-z\left(v_{0}^{1}\right)-\sum_{m=1}^{k} \frac{1}{m !} \sum_{I \in M_{m}} p_{I}\left(v_{0}^{m}\right) \tilde{x}_{I}, \\
\tilde{p}_{i} & =p_{i}-p_{i}\left(v_{0}^{1}\right)-\sum_{m=1}^{k-1} \frac{1}{m !} \sum_{I \in M_{m}} p_{I, i}\left(v_{0}^{m+1}\right) \tilde{x}_{I}(i=1, \cdots, n),
\end{aligned}
$$

where $\tilde{x}_{I}=x_{i_{1}} \cdots x_{i_{m}}$ for $I=\left(i_{1}, \cdots, i_{m}\right) \in M_{m}$.

q.e.d.

2.3. Canonical system $C^{k}$ on $J^{k}(\mathfrak{E})$. On a canonical coordinate neighborhood $U_{k}$ of $v_{0}^{k} \in J^{k}(\mathfrak{C})$, we denote by $\Gamma\left(U_{k}, C^{k}\right)$ the $\mathscr{R}\left(U_{k}\right)$-module of all sections of $C^{k}$ over $U_{k}$ and set

$$
\frac{\mathrm{d}^{(k)}}{\mathrm{d} x_{i}}=\frac{\partial}{\partial x_{i}}+p_{i} \frac{\partial}{\partial z}+\sum_{I \in \Sigma_{k-1}} p_{I, i} \frac{\partial}{\partial p_{I}}(i=1, \cdots, n) .
$$


Let $\{I\}$ denote the set $\left\{i, \cdots, i_{m}\right\}$ of integers $1, \cdots, n$ for $I=\left(i_{1}, \cdots, i_{m}\right)$. Then we have

Lemma 2.4. ( i ) $\frac{\mathrm{d}^{(k)}}{\mathrm{d} x_{1}}, \cdots, \frac{\mathrm{d}^{(k)}}{\mathrm{d} x_{n}}$ and $\frac{\partial}{\partial p_{I}}\left(I \in S_{k}\right)$

form a basis of $\Gamma\left(U_{k}, C^{k}\right)$.

(ii) For $i, j=1, \cdots, n$,

$$
\left[\frac{\mathrm{d}^{(k)}}{\mathrm{d} x_{i}}, \frac{\mathrm{d}^{(k)}}{\mathrm{d} x_{j}}\right]=\left[\frac{\partial}{\partial z}, \frac{\mathrm{d}^{(k)}}{\mathrm{d} x_{i}}\right]=0, \quad\left[\frac{\partial}{\partial p_{i}}, \frac{\mathrm{d}^{(k)}}{\mathrm{d} x_{j}}\right]=\delta_{j}^{i} \frac{\partial}{\partial z},
$$

where $\delta_{j}^{i}$ is the Kronecker's delta.

(iii) For each $I \in \Sigma_{k}$,

$$
\begin{array}{ll}
{\left[\frac{\partial}{\partial p_{I}}, \frac{\mathrm{d}^{(k)}}{\mathrm{d} x_{i}}\right]=0} & \text { if } i \notin\{I\}, \\
{\left[\frac{\partial}{\partial p_{I}}, \frac{\mathrm{d}^{(k)}}{\mathrm{d} x_{i}}\right]=\frac{\partial}{\partial p_{I(i)}}} & \text { if } i \in\{I\},
\end{array}
$$

where $I(i)$ is the unique element of $\Sigma_{k-1}$ such that $\{I(i), i\}=\{I\}$.

Hence, for $\xi_{I} \in \boldsymbol{R}\left(I \in M_{m}\right)$ such that $\xi_{I}$ is symmetric with respect to $I$,

$$
\left[\left[\cdots\left[\sum_{I \in S_{m}} \xi_{I} \frac{\partial}{\partial p_{I}}, \frac{\mathrm{d}^{(k)}}{\mathrm{d} x_{j_{1}}}\right], \cdots\right], \frac{\mathrm{d}^{(k)}}{\mathrm{d} x_{j_{m}}}\right]=\xi_{j_{1} \cdots j_{m}} \frac{\partial}{\partial z} .
$$

The proof is immediate, hence is omitted.

One should note that the vector fields $\mathrm{d}^{(k)} / \mathrm{d} x_{1}, \cdots, \mathrm{d}^{(k)} / \mathrm{d} x_{n}, \partial / \partial z$ and $\partial / \partial p_{I}$ $\left(I \in \Sigma_{k}\right)$ define an absolute parallelism on $U_{k}$ and form the dual basis of $\mathrm{d} x_{1}$, $\cdots, \mathrm{d} x_{n}, \hat{\pi}, \hat{\pi}_{I}$ and $\mathrm{d} p_{J}\left(I \in \Sigma_{k-1}, J \in S_{k}\right)$ at each point of $U_{k}$.

In this context, (ii) and (iii) of Lemma 2.4 are equivalent to the following structure equation;

$$
\begin{aligned}
\mathrm{d} \hat{\pi} & =\sum_{i=1}^{n} \omega_{i} \wedge \hat{\pi}_{i}, \\
\mathrm{~d} \hat{\pi}_{I} & =\sum_{j=1}^{n} \omega_{j} \wedge \hat{\pi}_{I, j}\left(I \in \Sigma_{k-1}\right), \\
\mathrm{d} \omega_{i} & =\mathrm{d} \hat{\pi}_{J}=0\left(i=1, \cdots, n, J \in S_{k}\right),
\end{aligned}
$$

where $\omega_{i}=\mathrm{d} x_{i}$ and $\hat{\pi}_{J}=\mathrm{d} p_{J}$.

Let $C_{m}^{k}$ and $Q_{m}^{k}$ denote differential systems on $J^{k}(\mathfrak{S})$ defined by

$$
\begin{array}{ll}
C_{m}^{k}=\left(\rho_{m}^{k}\right)_{*}^{-1}\left(C^{m}\right) & (m=1, \cdots, k), \\
Q_{m}^{k}=\left(\rho_{m+1}^{k}\right)_{*}^{-1}\left(Q^{m+1}\right) & (m=1, \cdots, k-1),
\end{array}
$$


where $Q^{m+1}=\operatorname{Ker}\left(\rho_{m}^{m+1}\right)_{*}$. Hence $Q_{m}^{k}=\operatorname{Ker}\left(\rho_{m}^{k}\right)_{*}$ and $Q^{k}=Q_{k-1}^{k}$.

Then, for $C_{m}^{k}$ and $Q_{m}^{k}$, we have

Proposition 2.5. (1) $C^{k}$ is a regular differential system such that $T\left(J^{k}(\Im)\right)=\partial^{(k)} C^{k}$ and $C_{m}^{k}=\partial^{(k-m)} C^{k}(m=1, \cdots, k-1)$, where $\partial^{(\alpha)} C^{k}$ is the $\alpha$-th weak derived system of $C^{k}$. Furthermore $C_{m}^{k}$ coincides with the first derived system $\partial C_{m+1}^{k}$ of $C_{m+1}^{k}$, i.e., $C_{m}^{k}=\partial^{k-m} C^{k}(m=1, \cdots, k-1)$, where $\partial^{\alpha} C^{k}$ is the $\alpha$-th derived system of $C^{k}$.

(2) $Q_{m}^{k}$ is a subbundle of $C_{m+1}^{k}$ of codimension $n$ and coincides with the Cauchy-Cartan characteristic system $C h\left(C_{m}^{k}\right)$ of $C_{m}^{k}(m=1, \cdots, k-1)$.

(3) If $k \geqq 3, Q_{m}^{k}$ coincides with $C_{m+1}^{k} \cap Q_{m-1}^{k}$ for $m=2, \cdots, k-1$, i.e., $Q_{m}^{k}\left(v^{k}\right)=C_{m+1}^{k}\left(v^{k}\right) \cap Q_{m-1}^{k}\left(v^{k}\right)$ for each $v^{k} \in J^{k}(\mathfrak{S})$. In particular $Q^{k}=C^{k} \cap Q_{1}^{k}$.

Proof. Matters being of local nature, it suffices to show the assertions

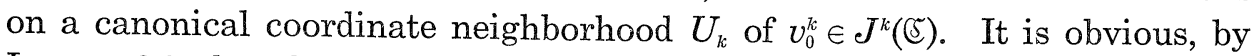
Lemma 2.3, that $C_{m}^{k}$ is defined on $U_{k}$ by $\hat{\pi}$ and $\hat{\pi}_{I}\left(I \in \Sigma_{m-1}\right)$ and that $Q_{m-1}^{k}$ is defined on $U_{k}$ by $\mathrm{d} x_{1}, \cdots, \mathrm{d} x_{n}, \hat{\pi}$ and $\hat{\pi}_{I}\left(I \in \Sigma_{m-1}\right)$. Hence $Q_{m-1}^{k}$ is a subbundle of $C_{m}^{k}$ of codimension $n$ and (3) holds.

(1) By Lemma 2.4 and the definition of weak derived systems, it is easy to see that $\partial^{(\alpha)} \mathscr{C}^{k}(\alpha=1, \cdots, k-1)$ defines a differential system such that $\partial^{(\alpha)} \mathscr{C}_{v^{k}}^{k}$ is generated by $\mathrm{d}^{(k)} / \mathrm{d} x_{1}, \cdots, \mathrm{d}^{(k)} / \mathrm{d} x_{n}$ and $\partial / \partial p_{I}\left(I \in \bigcup_{m=k-\alpha}^{k} S_{m}\right)$ at each $v^{k} \in U_{k}$. Hence $\partial^{(\alpha)} C^{k}$ is defined on $U_{k}$ by $\hat{\pi}$ and $\hat{\pi}_{I}\left(I \in \Sigma_{k-\alpha-1}\right)$. Then it follows that $\partial^{(\alpha)} C^{k}=C_{k-\alpha}^{k}(\alpha=1, \cdots, k-1)$. It is also clear that $T\left(J^{k}(\mathcal{C})\right)=$ $\partial^{(k)} C^{k}$. The last assertion follows immediately from Lemma 2.4.

(2) $C_{m}^{k}$ is defined on $U_{k}$ by $\hat{\pi}$ and $\hat{\pi}_{I}\left(I \in \Sigma_{m-1}\right)$. Furthermore, from (2.1), we get

$$
\begin{array}{ll}
\mathrm{d} \hat{\pi} \equiv \mathrm{d} \hat{\pi}_{I} \equiv 0\left(\bmod \hat{\pi}, \hat{\pi}_{J}\left(J \in \Sigma_{m-1}\right)\right) \quad \text { for } I \in \Sigma_{m-2}, \\
\mathrm{~d} \hat{\pi}_{I}=\sum_{j=1}^{n} \hat{\omega}_{j} \wedge \hat{\pi}_{I, j} \quad \text { for } I \in S_{m-1} .
\end{array}
$$

Hence, from Lemma 1.2, it follows that $C h\left(C_{m}^{k}\right)$ is defined on $U_{k}$ by $\mathrm{d} x_{1}, \cdots$, $\mathrm{d} x_{n}, \hat{\pi}$ and $\hat{\pi}_{I}\left(I \in \Sigma_{m}\right)$. Therefore we get $C h\left(C_{m}^{k}\right)=Q_{m+1}^{k}$ for $m=1, \cdots, k-1$.

q.e.d.

As for the differential system $\left(J^{k}(\mathfrak{S}), C^{k}\right)(k \geqq 2)$, one should observe the following: Let $\Lambda$ be a Lagrangian submanifold, i.e., an $n$-dimensional integral manifold, of 5 . We define an $n$-dimensional integral manifold $\Lambda^{k}$ of $\left(J^{k}(\mathfrak{E}), C^{k}\right)$ inductively as follows. $\Lambda^{m}$ being defined, let $\kappa_{m}$ be a map of $\Lambda^{m}$ into $J\left(J^{m}(\mathcal{S}), n\right)$ defined by $\kappa_{m}\left(\lambda^{m}\right)=T_{\lambda^{m}}\left(\Lambda^{m}\right)$ for $\lambda^{m} \in \Lambda^{m}$. Then, since $\Lambda^{m}$ is an $n$-dimensional integral manifold of $\left(J^{m}(\mathfrak{S}), C^{m}\right), \Lambda^{m+1}=\kappa_{m}\left(\Lambda^{m}\right)$ is an $n$-dimensional submanifold of $J^{m+1}(\mathfrak{5})$. Furthermore it is easy to see, by the definition of $C^{m+1}$, that $\Lambda^{m+1}$ is an integral manifold of $\left(J^{m+1}(\mathfrak{E}), C^{m+1}\right)$ such that $T_{\lambda^{m+1}}\left(\Lambda^{m+1}\right) \cap Q^{m+1}\left(\lambda^{m+1}\right)=\{0\}$ for $\lambda^{m+1} \in \Lambda^{m+1}$. Conversely, let $v^{k}$ be any point 
of $J^{k}(\mathfrak{S})$. Let $N$ be an $n$-dimensional integral manifold of $\left(J^{k}(\mathfrak{S}), C^{k}\right)$ such that $v^{k} \in N$ and $T_{v}(N) \cap Q^{k}(v)=\{0\}$ for $v \in N$. Then there exists a Lagrangian submanifold $\Lambda$ of $\mathcal{E}, v^{1}=\rho_{1}^{k}\left(v^{k}\right) \in \Lambda$, such that $N$ and $\Lambda^{k}$ coincides on a neighborhood of $v^{k}$.

On the other hand, by Proposition 2.5, we also notice that, since $Q^{k}$ is a subbundle of $C^{k}$, each fibre of $\rho_{k-1}^{k}: J^{k}(\mathfrak{S}) \rightarrow J^{k-1}(\mathfrak{S})$ is an integral manifold of $\left(J^{k}(\right.$ () $\left.), C^{k}\right)$.

However, $Q^{k}$ is intrinsically determined by $C^{k}$, i.e., $Q^{k}=C h\left(\partial C^{k}\right)$. In other words $Q^{k}$ is a covariant system of $C^{k}$ in the sense of E. Cartan (cf. Remark 1.4). Furthermore $Q^{k}$ is a subbundle of $C^{k}$ of codimension $n$.

In view of these facts, we can say that $\left(J^{k}(\mathfrak{S}), C^{k}\right)(k \geqq 2)$ is a differential system with (geometrically defined) $n$ independent variables. In particular $\left(J^{2}(\mathfrak{S}), C^{2}\right)=(L(\mathcal{E}), E)$ is a differential system with $n$ independent variables. This being remarked, $\left(J^{k}(\Subset), C^{k}\right)(k \geqq 3)$ corresponds to the usual $(k-2)$-th prolongation of $(L(5), E)$ (cf. [14, p. 94]).

\section{§ 3. Contact transformations and the symbol algebra of $\left(J^{k}(\mathfrak{S}), C^{k}\right)$}

3.1. Contact transformations. We will consider contact transformations of higher order. First we have

Proposition 3.1. Let $\mathbb{C}$ and $\hat{\mathbb{C}}$ be contact manifolds of dimension $2 n+1$, and let $\left(J^{k}(\mathfrak{C}), C^{k}\right)$ (resp. $\left.\left(J^{k}(\hat{\mathfrak{C}}), \hat{C}^{k}\right)\right)$ be the $k$-th order prolongation of $\mathfrak{\complement}$ (resp. $\hat{\mathfrak{S}})$. Let $\psi$ be an isomorphism of $\left(J^{k}(\mathfrak{C}), C^{k}\right)$ onto $\left(J^{k}(\hat{\mathfrak{C}}), \hat{C}^{k}\right)$. Then, for $k \geqq 1$, $\psi$ induces a unique isomorphism $\mathrm{q} \psi$ of $\left(J^{k+1}(\mathfrak{\subseteq}), C^{k+1}\right)$ onto $\left(J^{k+1}(\hat{\mathfrak{S}}), \hat{C}^{k+1}\right) d e-$ fined by $\mathrm{q} \psi\left(v^{k+1}\right)=\psi_{*}\left(v^{k+1}\right)$ for $v^{k+1} \in J^{k+1}(\mathfrak{S})$. Furthermore, for $k=2, \psi$ induces a unique isomorphism $\phi$ of $\left(J^{k-1}(\mathfrak{E}), C^{k-1}\right)$ onto $\left(J^{k-1}(\hat{\mathfrak{C}}), \hat{C}^{k-1}\right)$ such that $\psi=q \phi$.

Proof. First, since $\psi$ is a diffeomorphism of $J^{k}(\mathfrak{E})$ onto $J^{k}(\hat{\complement}), \psi$ induces the diffeomorphism $\mathrm{d} \psi$ (the differential of $\psi$ ) of $J\left(J^{k}(\mathfrak{E}), n\right)$ onto $J\left(J^{k}(\hat{\complement}), n\right)$ defined by $\mathrm{d} \psi(w)=\psi_{*}(w)$ for $w \in J\left(J^{k}(\mathfrak{C}), n\right)$. Since $\psi$ preserves the canonical systems, $d \psi$ sends $n$-dimensional integral elements of $\left(J^{k}(\mathfrak{S}), C^{k}\right)$ onto $n$ dimensional integral elements of $\left(J^{k}(\widehat{\complement}), \hat{C}^{k}\right)$. By Proposition 2.5, we have $Q^{k}=C h\left(\partial C^{k}\right)$ and $\hat{Q}^{k}=C h\left(\partial \hat{C}^{k}\right)$. Hence $\psi_{*}\left(Q^{k}\right)=\hat{Q}^{k}(k \geqq 2)$. Therefore we get $\mathrm{d} \psi\left(J^{k+1}(\mathfrak{S})\right)=J^{k+1}(\hat{\mathfrak{S}})(k \geqq 1)$. We set $\mathrm{q} \psi=\left.\mathrm{d} \psi\right|_{J^{k+1}(\mathbb{E})}$. Obviously we have $\hat{\rho}_{k}^{k+1} \cdot \mathrm{q} \psi=\psi \cdot \rho_{k}^{k+1}$. Then, by the definition of canonical systems, it is easy to see that $(q \psi)_{*}\left(C^{k+1}\right)=\hat{C}^{k+1}$.

Each fibre of $\rho_{k-1}^{k}: J^{k}(\mathfrak{E}) \rightarrow J^{k-1}(\mathfrak{E})$ (resp. $\left.\hat{\rho}_{k-1}^{k}: J^{k}(\hat{\mathfrak{S}}) \rightarrow J^{k-1}(\hat{\mathfrak{C}})\right)$ is connected, hence is a maximal integral manifold of $Q^{k}=C h\left(\partial C^{k}\right)\left(\operatorname{resp} . \hat{Q}^{k}=C h\left(\partial \hat{C}^{k}\right)\right)$ $(k \geqq 2)$. Hence, from $\psi_{*}\left(Q^{k}\right)=\hat{Q}^{k}$, we see that $\psi$ is fibre-preserving. Therefore $\psi$ induces a unique diffeomorphism $\phi$ of $J^{k-1}(\mathfrak{S})$ onto $J^{k-1}(\hat{\mathfrak{C}})$ such that $\hat{\rho}_{k-1}^{k} \cdot \psi=\phi \cdot \rho_{k-1}^{k}$. By Proposition 2.5, we have $\left(\rho_{k-1}^{k}\right)_{*}^{-1}\left(C^{k-1}\right)=\partial C^{k}$ and $\left(\hat{\rho}_{k-1}^{k}\right)_{*}^{-1}$ 
$\left(\hat{C}^{k-1}\right)=\partial \hat{C}^{k}$. Then, from $\psi_{*}\left(C^{k}\right)=\hat{C}^{k}$, we get $\phi_{*}\left(C^{k-1}\right)=\hat{C}^{k-1}$. Hence $\phi$ is an isomorphism of $\left(J^{k-1}(\mathfrak{S}), C^{k-1}\right)$ onto $\left(J^{k-1}(\widehat{\mathfrak{C}}), \hat{C}^{k-1}\right)$.

It remains to show $\psi=\mathrm{q} \phi$. For this purpose, considering $(\mathrm{q} \phi)^{-1} \cdot \psi$, we have only to show that $\tilde{\psi}=i d_{J^{k}(\mathbb{E})}$ if $\tilde{\psi}$ is an isomorphism of $\left(J^{k}(\mathcal{E}), C^{k}\right)$ onto itself such that $\rho_{k-1}^{k} \cdot \tilde{\psi}=\rho_{k-1}^{k}$. Let $v^{k}$ be any point of $J^{k}(\mathfrak{S})$. From $\tilde{\psi}_{*}\left(C^{k}\right)=C^{k}$, we have $\tilde{\psi}_{*}\left(C^{k}\left(v^{k}\right)\right)=C^{k}\left(\tilde{\psi}\left(v^{k}\right)\right)$. On the other hand, by the definition of the canonical system $C^{k}$, we have $\left(\rho_{k-1}^{k}\right) *\left(C^{k}\left(v^{k}\right)\right)=v^{k}$. Hence, from $\rho_{k-1}^{k} \cdot \tilde{\psi}=\rho_{k-1}^{k}$, we get

$$
\tilde{\psi}\left(v^{k}\right)=\left(\rho_{k-1}^{k}\right)_{*}\left(C^{k}\left(\tilde{\psi}\left(v^{k}\right)\right)\right)=\left(\rho_{k-1}^{k}\right)_{*} \cdot \tilde{\psi}_{*}\left(C^{k}\left(v^{k}\right)\right)=v^{k} .
$$

Thus we get $\tilde{\psi}=i d_{J^{k}(\Subset)}$.

q.e.d.

By Proposition 3.1, we obtain

TheOREm 3.2. Let $\mathbb{S}$ and $\hat{\mathfrak{S}}$ be contact manifolds of dimension $2 n+1$, and let $\left(J^{k}(\mathfrak{S}), C^{k}\right)\left(\right.$ resp. $\left.\left(J^{k}(\hat{\mathbb{C}}), \hat{C}^{k}\right)\right)$ be the $k$-th order prolongation of $\mathbb{S}$ (resp. $\left.\hat{\mathbb{S}}\right)$. Then a contact transformation $\phi$ of $\mathfrak{E}$ onto $\hat{\mathfrak{E}}$, i.e., $\phi:\left(J^{1}(\mathfrak{E}), C^{1}\right) \rightarrow\left(J^{1}(\hat{\mathbb{C}}), \hat{C}^{1}\right)$ isomorphism, induces a unique isomorphism $\mathrm{q}^{k} \phi$ of $\left(J^{k}(\mathfrak{S}), C^{k}\right)$ onto $\left(J^{k}(\widehat{\mathbb{C}}), \hat{C}^{k}\right)$ such that $\mathrm{q}^{k} \phi\left(v^{k}\right)=\left(\mathrm{q}^{k-1} \phi\right)_{*}\left(v^{k}\right)$ for $v^{k} \in J^{k}(\mathbb{C})(k \geqq 2)$, where we set $\mathrm{q}^{1} \phi=\phi$ by convention.

Conversely an isomorphism \& of $\left(J^{k}(\mathfrak{C}), C^{k}\right)$ onto $\left(J^{k}(\hat{\mathbb{C}}), \hat{C}^{k}\right)(k \geqq 2)$ induces a unique contact transformation $\phi$ of $\mathbb{E}$ onto $\hat{\mathbb{E}}$ such that $\psi=\mathrm{q}^{k} \phi$.

Hence an isomorphism of $\left(J^{k}(\mathcal{E}), C^{k}\right)$ onto $\left(J^{k}(\hat{\mathbb{C}}), \hat{C}^{k}\right)$ may be called a contact transformation of $k$-th order. And $\mathrm{q}^{k} \phi$ is called the $k$-th order prolongation of $\phi$.

Theorem 3.2 is, in its local form, due to A. V. Bäcklund [3] (cf. [1]).

REMARK 3.3. Let $(M, N, p)$ be a fibred manifold of fibre dimension 1, i.e., $p$ is a submersion of $M$ onto $N$ such that $\operatorname{dim} N=n$ and $\operatorname{dim} M=n+1$. Let $J^{k}(M, N, p)$ be the bundle of $k$-jets of local cross-sections of $(M, N, p)$ (cf. [9] or [15]). $\quad J^{1}(M, N, p)$ has the canonical contact structure $C^{1}$ (Proposition 1 $[14, \mathrm{p} .85])$. We denote by (5 the contact manifold $\left(J^{1}(M, N, p), C^{1}\right)$ of dimension $2 n+1$. Let $J\left(J^{k}(M, N, p), n\right)$ be the Grassmann bundle of $n$-dimensional contact elements of $J(M, N, p)$ with the projection $\Pi_{k}: J\left(J^{k}(M, N, p), n\right) \rightarrow$ $J^{k}(M, N, p)$. Then, as is well known, $J^{k}(M, N, p)$ can be regarded as a submanifold of $J\left(J^{k-1}(M, N, p), n\right)$ as follows: Let $z \in J^{k}(M, N, p)$ and $x=p_{-1}^{k}(z) \in$ $N$. Let $f$ be a local section of $(M, N, p)$ such that $z=j_{x}^{k}(f)$. Then the imbed$\operatorname{ding} \tilde{\imath}^{k}$ of $J^{k}(M, N, p)$ into $J\left(J^{k-1}(M, N, p), n\right)$ is defined by $\tilde{\iota}^{k}(z)=\left(j^{k-1}\right)_{*}\left(T_{x}(N)\right)$, where $j^{k-1}(f)$ is the local section of $\left(J^{k-1}(M, N, p), N, p_{-1}^{k-1}\right)$ induced by $f . \quad \tau^{k}$ is well-defined and satisfies $\Pi_{k-1} \cdot \tilde{\iota}^{k}=p_{k-1}^{k}$.

First one should note that $\tilde{\iota}^{2}\left(J^{2}(M, N, p)\right) \subset J^{2}(\Im)$ and $\iota^{2}: J^{2}(M, N, p) \rightarrow J^{2}(\Im)$ is an open imbedding. Then, inductively, it is easy to see that $\tilde{\iota}^{k}$ induces the 
open imbedding $\iota^{k}$ of $J^{k}(M, N, p)$ into $J^{k}(\Subset)$ satisfying the following commutative diagram $(k \geqq 3)$;

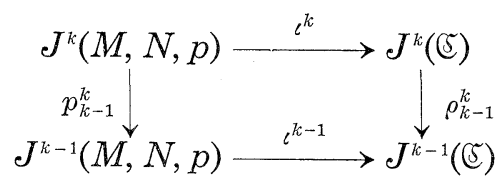

Furthermore, it is easy to see that $\iota^{k}\left(J^{k}(M, N, p)\right)=\left(\rho_{2}^{k}\right)^{-1}\left(\iota^{2}\left(J^{2}(M, N, p)\right)\right)(k \geqq 2)$. In other words, $\left(J^{k+1}(M, N, p), J^{k}(M, N, p), p_{k}^{k+1}\right)$ is the induced bundle of $\left(J^{k+1}(\mathfrak{C}), J^{k}(\mathfrak{C}), \rho_{k}^{k+1}\right)$ by $\iota^{k}$ for $k \geqq 2$. On the other hand we may say that $\left(J^{2}(\mathfrak{S}), J^{1}(M, N, p), \rho_{1}^{2}\right)$ is obtained by a suitable compactification of fibres of $\left(J^{2}(M, N, p), J^{1}(M, N, p), p_{1}^{2}\right)$. This compactification is needed in order to lift the action of contact transformations.

$J^{k}(M, N, p)$ has the canonical system $\bar{C}^{k}$ such that $\bar{C}^{k}=\left(\varepsilon^{k}\right)_{*}^{-1}\left(C^{k}\right)$. For an isomorphism $\psi$ of $\left(J^{k}(M, N, p), \bar{C}^{k}\right)(k \geqq 2)$, it can be shown that $\psi$ is the natural lift of an isomorphism $h$ of $(M, N, p)$, provided that each fibre of $p$; $M \rightarrow N$ is connected. For the detail of this fact, we refer to [21].

3.2. Symbol algebra of $\left(J^{k}(\mathfrak{\complement}), C^{k}\right)$. Let $v_{0}^{k}$ be any point of $J^{k}(\mathfrak{S})$, and let $\left(x_{i}, z, p_{I}\right)\left(i=1, \cdots, n, I \in \Sigma_{k}\right)$ be a canonical coordinate system on a neighborhood $U_{k}$ of $v_{0}^{k}$ (Lemma 2.3).

Let $\mathfrak{X}\left(U_{k}\right)$ be the Lie algebra of all vector fields on $U_{k}$. Let $c\left(U_{k}\right)$ be the linear subspace of $\mathfrak{X}\left(U_{k}\right)$ spanned by $N(k)+n+1$ vector fields $\mathrm{d}^{(k)} / \mathrm{d} x_{1}, \cdots$, $\mathrm{d}^{(k)} / \mathrm{d} x_{n}, \partial / \partial z$ and $\partial / \partial p_{I}\left(I \in \Sigma_{k}\right)$. Then, from Lemma 2.4, it follows that $c\left(U_{k}\right)$ is a subalgebra of $\mathfrak{X}\left(U_{k}\right)$ with the structure equation (2.1). Furthermore $c\left(U_{k}\right)$ has a gradation given by

where

$$
\begin{gathered}
c\left(U_{k}\right)=\sum_{p<0} \mathfrak{c}_{p}\left(U_{k}\right) \text { (direct sum), } \\
\mathfrak{c}_{-1}\left(U_{k}\right)=\left\langle\left\{\frac{\mathrm{d}^{(k)}}{\mathrm{d} x_{1}}, \cdots, \frac{\mathrm{d}^{(k)}}{\mathrm{d} x_{n}}, \frac{\partial}{\partial p_{I}}\left(I \in S_{k}\right)\right\}\right\rangle, \\
\mathfrak{c}_{p}\left(U_{k}\right)=\left\langle\left\{\frac{\partial}{\partial p_{I}}\left(I \in S_{p+k+1}\right)\right\}\right\rangle(-k \leqq p \leqq-2), \\
\mathfrak{c}_{-(k+1)}\left(U_{k}\right)=\left\langle\left\{\frac{\partial}{\partial z}\right\}\right\rangle, \\
\mathfrak{c}_{p}(U)=\{0\} \quad \text { if } p<-(k+1),
\end{gathered}
$$

and $\langle\{\}\rangle$ denotes the linear subspace spanned by the elements of \{\} . With this gradation, $c\left(U_{k}\right)$ is a fundamental graded algebra of $(k+1)$-th kind (cf. Definition 1.3 [19]).

Now we consider the graded algebra $c^{k}(v)$ of $\left(J^{k}(\mathfrak{E}), C^{k}\right)$ at $v \in U_{k}$. First we have 
Lemma 3.4. Let $v$ be any point of $U_{k}$. Then

(i ) $\left(C^{k}\right)^{p}(v)=\left\{X_{v} \in T_{v}\left(J^{k}(5)\right) \mid X \in \sum_{q \geqq p} c_{q}\left(U_{k}\right)\right\}$ for $-(k+1) \leqq p<0$, where $\left(C^{k}\right)^{p}=\partial^{(-p-1)} C^{k}(p<-1)$ and $\left(C^{k}\right)^{-1}=C^{k}$.

(ii) The graded algebra $c^{k}(v)$ of $\left(J^{k}(\mathfrak{S}), C^{k}\right)$ at $v$ is isomorphic with $c\left(U_{k}\right)$.

(iii) $\left(J^{k}(\mathfrak{S}), C^{k}\right)$ is locally isomorphic with the standard differential system of type $\mathrm{c}\left(U_{k}\right)$.

Proof. ( $\mathrm{i}$ ) is equivalent to (1) of Proposition 2.5.

(ii) Recall that $c^{k}(v)=\sum_{p=-1}^{-(k+1)} c_{p}^{k}(v)$ (direct sum), where $\mathfrak{c}_{-1}(v)=C^{k}(v)$ and $c_{p}^{k}(v)=\left(C^{k}\right)^{p}(v) /\left(C^{k}\right)^{p+1}(v)(-(k+1) \leqq p \leqq-2)$. Let $\nu_{p}(v)$ be the linear isomorphism of $\mathfrak{c}_{p}\left(U_{k}\right)$ onto $c_{p}^{k}(v)$ defined by $\nu_{p}(v)(X)=\pi_{p}\left(X_{v}\right)$ for $X \in \mathfrak{c}_{p}\left(U_{k}\right)$, where $\pi_{p}$ is the projection of $\left(C^{k}\right)^{p}(v)$ onto $c_{p}^{k}(v)$. Then $\nu(v)=\nu_{-(k+1)}(v) \times \cdots \times \nu_{-1}(v)$ is a linear isomorphism of $c\left(U_{k}\right)$ onto $c^{k}(v)$. By the very definition of the bracket operation of $c^{k}(v)$ (1.3 of $\left.\S 1\right), \nu(v)$ is an isomorphism of graded algebras.

(iii) We consider $R^{N_{0}}$ with the natural coordinate $\left(x_{i}, z, p_{I}\right)(i=1, \cdots, n$, $\left.I \in \Sigma_{k}\right)$, where $N_{0}=N(k)+n+1$. Then $\mathrm{d}^{(k)} / \mathrm{d} x_{i}, \partial / \partial z$ and $\partial / \partial p_{I}(i=1, \cdots, n$, $\left.I \in \Sigma_{k}\right)$, define an absolute parallelism on $\boldsymbol{R}^{N_{0}}$ and generate an $N_{0}$-dimensional subalgebra $c\left(\boldsymbol{R}^{N_{0}}\right)$ of $\mathfrak{X}\left(\boldsymbol{R}^{N_{0}}\right)$. Obviously $c\left(\boldsymbol{R}^{N_{0}}\right)$ is isomorphic with $c\left(U_{k}\right)$. Furthermore, it is easy to see that each $X \in \mathfrak{c}\left(\boldsymbol{R}^{N_{0}}\right)$ is a complete vector field on $\boldsymbol{R}^{N_{0}}$. Let $M\left(\mathfrak{c}\left(U_{k}\right)\right)$ be the simply connected Lie group with Lie algebra $\mathfrak{c}\left(U_{k}\right)$. Then it is well known that there exists a diffeomorphism $\psi$ of $\boldsymbol{R}^{N_{0}}$ onto $M\left(\mathfrak{c}\left(U_{k}\right)\right)$ such that $\psi_{*}(X)$ is a left invariant vector field for each $X \in \mathfrak{c}\left(\boldsymbol{R}^{N_{0}}\right)$ (cf. Theorem VIII [17, p. 105]). Therefore $\left(J^{k}(\mathfrak{E}), C^{k}\right)$ is locally isomorphic with the standard differential system of type $c\left(U_{k}\right)$ (cf. 2 [19]). q.e.d.

We will describe the structure of $c\left(U_{k}\right)$ in the following way: Let $V$ and $W$ be vector spaces over $\boldsymbol{R}$ of dimension $n$ and $m$ respectively. Let $V^{*}$ denote the dual space of $V$ and $S^{r}\left(V^{*}\right)$ denote the $r$-th symmetric product of $V^{*}$. By convention we set $S^{0}\left(V^{*}\right)=\boldsymbol{R}$ and $S^{1}\left(V^{*}\right)=V^{*}$. We define the $m \times N(k)+m+n$ dimensional vector space $\mathcal{C}^{k}(V, W)$ as follows:

$$
\mathfrak{S}^{k}(V, W)=\sum_{p<0} \Im_{p}^{k}(V, W)(\text { direct sum })
$$

where $\mathfrak{\Im}_{-(k+1)}^{k}(V, W)=W, \mathfrak{夭}_{p}^{k}(V, W)=W \otimes S^{p+k+1}\left(V^{*}\right)(-k \leqq p \leqq-2), \widetilde{\mho}(V, W)$ $=W \otimes S^{k}\left(V^{*}\right), \mathfrak{\complement}_{-1}^{k}(V, W)=V \oplus \widetilde{\jmath}(V, W)$ (direct sum) and $\mathfrak{\complement}_{p}^{k}(V, W)=\{0\}$ if $p<-(k+1)$.

The bracket operation of $\mathfrak{C}^{k}(V, W)$ is defined as follows:

$$
\begin{aligned}
& {[W, V]=\{0\}, \quad[V, V]=\{0\},} \\
& {\left[W \otimes S^{r}\left(V^{*}\right), W \otimes S^{s}\left(V^{*}\right)\right]=\{0\}(r, s=0, \cdots, k),} \\
& \left.\left[W \otimes S^{r}\left(V^{*}\right), V\right]=W \otimes(V\lrcorner S^{r}\left(V^{*}\right)\right)(r=1, \cdots, k),
\end{aligned}
$$

i.e., $\left.\left[w \otimes s^{r}, v\right]=w \otimes(v\lrcorner s^{r}\right)$ for $v \in V, w \in W$ and $s^{r} \in S^{r}\left(V^{*}\right)$, where $\lrcorner$ is the 
interior multiplication. Hence $\left[\left[\cdots\left[w \otimes s^{r}, v_{1}\right], \cdots\right], v_{r}\right]=s^{r}\left(v_{1} \bigcirc \cdots \odot v_{r}\right) w \in$ $W=\mathfrak{C}_{-(k+1)}^{k}(V, W)$ for $w \in W, s^{r} \in S^{r}\left(V^{*}\right)$ and $v_{i} \in V(i=1, \cdots, r)$. Furthermore one should note

$$
\widetilde{F}(V, W)=\left\{X \in \mathfrak{C}_{-1}^{k}(V, W) \mid\left[X, \mathfrak{\complement}_{p}^{k}(V, W)\right]=0\right\} \quad \text { for }-k \leqq p \leqq-2 .
$$

Equipped with this bracket operation, $\mathcal{S}^{k}(V, W)$ is a fundamental graded algebra of $(k+1)$-th kind. In case $\operatorname{dim} W=1$, we denote $\mathbb{C}^{k}(V, W)$ simply by $\mathfrak{C}^{k}(n)$. Then $\mathfrak{E}^{k}(n)$ is isomorphic with $\mathfrak{c}\left(U_{k}\right)$. In fact, take a basis $w_{0}$ of $W$ and a basis $e_{1}, \cdots, e_{n}$ of $V$ and let $e_{1}^{*}, \cdots, e_{n}^{*}$ be its dual basis of $V^{*}$. Then $e_{I}=$ $e_{i_{1}}\left(\right.$ () $\cdots\left(e_{i_{r}}\left(I=\left(i_{1}, \cdots, i_{r}\right) \in S_{r}\right)\right.$ form a basis of $S^{r}(V)$. The dual basis $\left\{e_{I}^{*}\right\}_{I \in S_{r}}$ of $\left\{e_{I}\right\}_{I \in S_{r}}$ is given by

$$
e_{I}^{*}=\frac{r !}{\alpha_{1} ! \cdots \alpha_{n} !} e_{i_{1}}^{*}\left(\cdots \odot e_{i_{r}}^{*} \quad \text { for } I=\left(i_{1}, \cdots, i_{r}\right) \in S_{r},\right.
$$

where $\left(\alpha_{1}, \cdots, \alpha_{n}\right)$ is the multi-index of $I$, i.e., $\alpha_{1}+\cdots+\alpha_{n}=r$ and $\partial^{r} / \partial x_{i_{1}} \cdots$ $\partial x_{i_{r}}=\partial^{r} / \partial x_{1}^{\alpha_{1}} \cdots \partial x_{n}^{\alpha_{n}}$. These being remarked, let $\nu_{0}$ be a linear isomorphism of $\mathfrak{C}^{k}(n)$ onto $\mathrm{c}\left(U_{k}\right)$ defined by $\nu_{0}\left(w_{0}\right)=\partial / \partial z, \nu_{0}\left(e_{i}\right)=\mathrm{d}^{(k)} / \mathrm{d} x_{i}(i=1, \cdots, n)$ and $\nu_{0}\left(w_{0} \otimes e_{I}^{*}\right)=\partial / \partial p_{I}\left(I \in \Sigma_{k}\right)$. Hence, for $\xi_{I} \in R\left(I \in S_{r}\right)$ such that $\xi_{I}$ is symmetric with respect to $I=\left(i_{1}, \cdots, i_{r}\right)$, we have

$$
\left.\nu_{0}\left(\sum_{I \in M_{r}} \xi_{I} w_{0} \otimes e_{i_{1}}^{*} \otimes \cdots e_{i_{r}}^{*}\right)\right)=\sum_{I \in S_{r}} \xi_{I} \frac{\partial}{\partial p_{I}} .
$$

Then, from Lemma 2.4, it follows immediately that $\nu_{0}$ is an isomorphism of graded algebras. And the structure equation of $\mathbb{C}^{k}(n)$ is given by (2.1).

Definition 3.5. Notations being as above, the fundamental graded algebra $\mathbb{C}^{k}(V, W)$ is called the contact algebra of $k$-th order of bidegree $(n, m)$, where $n=\operatorname{dim} V$ and $m=\operatorname{dim} W$. Furthermore, $\mathfrak{S}^{k}(n)$ is simply called the contact algebra of $k$-th order of degree $n$.

Summarizing the discussion above, we obtain

Proposition 3.6. Let \& be a contact manifold of dimension $2 n+1$. Then $\left(J^{k}(\mathfrak{\complement}), C^{k}\right)$ is a regular differential system of type $\mathfrak{S}^{k}(n)$. Furthermore, $\left(J^{k}(\mathfrak{S})\right.$, $\left.C^{k}\right)$ is locally isomorphic with the standard differential system of type $\overbrace{}^{k}(n)$.

Next we will study the structure of the group $A^{k}(V, W)$ of graded Lie algebra automorphisms of $\mathfrak{\complement}^{k}(V, W)$. For brevity, we write $\mathfrak{\Im}_{p}^{k}=\mathfrak{\Im}_{p}^{k}(V, W)$ and $\widetilde{F}=\widetilde{F}(V, W)$ in the following. Let $\kappa$ be the projection of $\mathfrak{C}_{-1}^{k}$ onto $V_{c}=\mathfrak{\complement}_{-1}^{k} / \widetilde{F}$. Then $\kappa_{0}=\left.\kappa\right|_{V}$ is a linear isomorphism of $V$ onto $V_{c}$. From $\mathfrak{F}=\left\{X \in \mathfrak{\mho}_{-1}^{k} \mid\left[X, \mathfrak{\mho}_{-2}^{k}\right]\right.$ $=0\}(k \geqq 2)$, it follows that $\phi(\widetilde{\gamma})=\widetilde{F}$ for $\phi \in A^{k}(V, W)$. Hence $\phi$ induces a unique linear isomorphism $\hat{\phi}$ of $V_{c}$ onto $V_{c}$ such that $\hat{\phi} \cdot \kappa=\kappa \cdot \phi$. We define the closed normal subgroup $N^{k}(V, W)$ of $A^{k}(V, W)$ by setting 


$$
N^{k}(V, W)=\left\{\phi \in A^{k}(V, W)|\phi|_{W}=i d_{W} \quad \text { and } \quad \hat{\phi}=i d_{V_{c}}\right\} .
$$

Let $\chi_{r}(r=1, \cdots, k)$ be the representation of $G L(V) \times G L(W)$ into $G L(W \otimes$ $S^{r}\left(V^{*}\right)$ ) defined by $\chi_{r}(a, b)=b \otimes \otimes^{r}\left(a^{*}\right)^{-1}$ for $a \in G L(V)$ and $b \in G L(W)$, where $a^{*}$ is the adjoint linear map of $a$. We define the isomorphism $\chi$ of $G L(V) \times$ $G L(W)$ into $A^{k}(V, W)$ by setting

$$
\left.\chi(a, b)\right|_{V}=a,\left.\chi(a, b)\right|_{W}=b \quad \text { and }\left.\quad \chi(a, b)\right|_{W \otimes S^{r}\left(V^{*}\right)}=\chi_{r}(a, b)
$$

for $r=1, \cdots, k$. And we set $G^{k}(V, W)=\chi(G L(V) \times G L(W))$. Let $\mu$ be the representation of $A^{k}(V, W)$ into $G L(\widetilde{F})$ defined by $\mu(\phi)=\left.\phi\right|_{\mathfrak{F}}$ for $\phi \in A^{k}(V, W)$. Furthermore, let $S^{k+1}(V, W)$ be the set of abelian subalgebras $\hat{V}$ of $\mathbb{C}^{k}$ such that $\mathfrak{C}_{-1}^{k}=\hat{V} \oplus \widetilde{\mho}$ (direct sum). Then we have

Proposition 3.7. Let $A^{k}(V, W)$ be the group of graded Lie algebra automorphisms of $\mathfrak{S}^{k}(V, W)(k \geqq 2)$. Let $N=N^{k}(V, W), G=G^{k}(V, W), S^{k+1}(V, W)$ and $\mu$ be as above.

(1) $N$ is a closed normal subgroup of $A^{k}(V, W)$ and is canonically isomorphic with the vector group $W \otimes S^{k+1}\left(V^{*}\right)$. Furthermore $N$ acts simply transitively on $S^{k+1}(V, W)$.

(2) $G=\left\{\phi \in A^{k}(V, W) \mid \phi(V)=V\right\}$ and $A^{k}(V, W)=G \cdot N$ (semi-direct product).

(3) $\operatorname{Ker} \mu=\chi\left(\operatorname{Ker} \chi_{k}\right) \cdot N$.

Proof. (1) Let $\phi$ be any element of $N$. We set $\rho_{\phi}(X)=\phi(X)-X$ for $X \in V$. Then, from $\hat{\phi}=i d_{V_{c}}$, it follows that $\rho_{\phi}$ is a linear map of $V$ into $\widetilde{F}$. Since $\phi$ is a homomorphism, we get

$$
[\phi(X), \phi(Y)]=Y\lrcorner \rho_{\phi}(X)-X \dashv \rho_{\phi}(Y)=0 \quad \text { for } X, Y \in V .
$$

Hence $\rho_{\phi}$ can be considered as an element of $W \otimes S^{k+1}\left(V^{*}\right)$. Furthermore, let $s^{r}$ be any vector of $W \otimes S^{r}\left(V^{*}\right)$. Then, by $\left.\phi\right|_{W}=i d_{W}$, we have

$$
\begin{aligned}
s^{r}\left(X_{1}, \cdots, X_{r}\right) & =\left[\cdots\left[s^{r}, X_{1}\right], \cdots, X_{r}\right] \\
& =\left[\cdots\left[\phi\left(s^{r}\right), \phi\left(X_{1}\right)\right], \cdots, \phi\left(X_{r}\right)\right],
\end{aligned}
$$

for $X_{i} \in V(i=1, \cdots, r)$. In the other hand, we have, $\left[W \otimes S^{r}\left(V^{*}\right), W \otimes S^{k}\left(V^{*}\right)\right]$ $=\{0\}$ for $r=1, \cdots, k$. Hence, from $\phi(X)=X+\rho_{\phi}(X)$ for $X \in V$, we get

$$
s^{r}\left(X_{1}, \cdots, X_{r}\right)=\left[\cdots\left[\phi\left(s^{r}\right), X_{1}\right], \cdots, X_{r}\right]=\phi\left(s^{r}\right)\left(X_{1}, \cdots, X_{r}\right) .
$$

Therefore, we obtain $\left.\phi\right|_{W \otimes S^{r}\left(V^{*}\right)}=i d_{W \otimes S^{r}\left(V^{*}\right)}$ for $r=1, \cdots, k$.

Conversely, let $\rho$ be a linear map of $V$ into $W \otimes S^{k}\left(V^{*}\right)$ such that $\left.X\right\lrcorner \rho(Y)$ $=Y\lrcorner \rho(X)$ for $X, Y \in V$, i.e., $\rho \in W \otimes S^{k+1}\left(V^{*}\right)$. Let $A_{\rho}$ be the linear isomorphism of $\mathbb{C}^{k}$ onto $\mathfrak{C}^{k}$ defined by 


$$
\begin{aligned}
& \left.A_{\rho}\right|_{W}=i d_{W},\left.A_{\rho}\right|_{V}=i d_{V}+\rho, \\
& \left.A_{\rho}\right|_{W \otimes S^{r}\left(V^{*}\right)}=i d_{W \otimes S^{r}} \quad \text { for } r=1, \cdots, k .
\end{aligned}
$$

Then we easily"get? $A_{\rho} \in N$. Thus we obtain

$$
N=\left\{A_{\rho} \in A^{k}(V, W) \mid \rho \in W \otimes S^{k+1}\left(V^{*}\right)\right\} .
$$

It is easy to see that $\rho \in W \otimes S^{k+1}\left(V^{*}\right) \mapsto A_{\rho} \in N$ is a group isomorphism.

Since $\phi(\widetilde{\mho})=\widetilde{\mho}$ for $\phi \in A^{k}(V, W), N$ acts on $S^{k+1}(V, W)$. Let $\hat{V}$ be any element of $S^{k+1}(V, W)$. Then $\hat{\kappa}=\left.\kappa\right|_{\hat{v}}$ is a linear isomorphism of $\hat{V}$ onto $V_{c}$. We define a linear isomorphism $\hat{\psi}$ of $\mathfrak{\complement}^{k}$ onto $\mathfrak{C}^{k}$ by setting

$$
\begin{aligned}
& \left.\hat{\psi}\right|_{W}=i d_{W},\left.\quad \hat{\psi}\right|_{V}=\hat{\kappa}^{-1} \cdot \kappa_{0}, \\
& \left.\hat{\psi}\right|_{W \otimes S^{r}\left(V^{*}\right)}=i d_{W \otimes S^{r}\left(V^{*}\right)} \quad \text { for } r=1, \cdots, k .
\end{aligned}
$$

Then it is easy to see that $\hat{\psi} \in N$ and $\hat{\psi}(V)=\hat{V}$. Hence $N$ acts transitively on $S^{k+1}(V, W)$. Furthermore, from (3.1), it follows that if $\phi \in N$ and $\phi(V)=V$, then $\phi=i d_{\mathbb{E}^{k}}$. Therefore, $N$ acts simply transitively on $S^{k+1}(V, W)$.

(2) Clearly we have $G \subset \hat{G}=\left\{\phi \in A^{k}(V, W) \mid \phi(V)=V\right\}$. Let $\phi$ be any element of $\hat{G}$. We set $\phi^{\prime}=\chi(a, b)^{-1} \cdot \phi$, where $a=\left.\phi\right|_{V} \in G L(V)$ and $b=\left.\phi\right|_{W} \in$ $G L(W)$. Then $\left.\phi^{\prime}\right|_{V}=i d_{V}$ and $\left.\phi^{\prime}\right|_{W}=i d_{W}$. In particular, $\phi^{\prime} \in N$ and $\phi^{\prime}(V)=V$. Hence we get $\phi^{\prime}=i d_{\mathbb{E}^{k}}$. Thus we obtain $G=\hat{G}$ and $G \cap N=\left\{i d_{\mathbb{\aleph}^{k}}\right\}$. Furthermore, let $\psi$ be any element of $A^{k}(V, W)$. We set $\psi^{\prime}=\chi(a, b)^{-1} \cdot \psi$, where $a=$ $\kappa_{0}^{-1} \cdot \hat{\psi} \cdot \kappa_{0} \in G L(V)$ and $b=\left.\psi\right|_{W} \in G L(W)$. Then we get easily $\psi^{\prime} \in N$. Therefore $A^{k}(V, W)$ is a semi-direct product of $G$ and $N$.

(3) By (3.1), we have $N \subset \operatorname{Ker} \mu$. Hence, by (2), we obtain $\operatorname{Ker} \mu=$ $\left(\operatorname{Ker} \chi^{k}\right) \cdot N$, where $\operatorname{Ker} \chi^{k}=\left\{\left(\lambda \cdot i d_{V}, \lambda^{k} \cdot i d_{W}\right) \in G L(V) \times G L(W) \mid \lambda \in R\right\}$. q.e.d.

3.3. Fundamental identification of $Q^{k}$. Let $v$ be any point of $J^{k}(\sqrt{ })$ and let $w$ be a point of $J^{k+1}(\mathbb{S})$ such that $\rho_{k}^{k+1}(w)=v$. Then $w=E$ is a subspace of $C^{k}(v)$ such that $C^{k}(v)=E \oplus Q^{k}(v)$ (direct sum). Being linear subspaces of $C^{k}(v)$ $=c_{-1}^{k}(v), E$ and $Q^{k}(v)$ are subspaces of $c^{k}(v)$. Let $\tilde{\alpha}_{p}(-k \leqq p \leqq-1)$ be the bilinear map of $c_{p}^{k}(v) \times \otimes^{p+k+1} c_{-1}^{k}(v)$ into $c_{-(k+1)}^{k}(v)$ defined by

$$
\tilde{\alpha}_{p}\left(Y_{p}, X_{1}, \cdots, X_{p+k+1}\right)=\left[\left[\cdots\left[Y_{p}, X_{1}\right], \cdots\right], X_{p+k+1}\right] \in \mathfrak{c}_{-(k+1)}^{k}(v)
$$

for $Y_{p} \in \mathfrak{c}_{p}^{k}(v)$ and $X_{i} \in \mathfrak{c}_{-1}^{k}(v)(i=1, \cdots, p+k+1)$. Let $V(v)$ denote the factor space $c_{-1}^{k}(v) / Q^{k}(v)$ and let $\kappa$ be the projection of $c_{-1}^{k}(v)$ onto $V(v)$.

LEMma 3.8. Notations being as above, we have

(i ) $\left[c_{p}^{k}(v), c_{-1}^{k}(v)\right]=c_{p-1}^{k}(v)$ and $\left[c_{p}^{k}(v), c_{q}^{k}(v)\right]=\{0\}$ if $p, q<-1$.

(ii) If $Y_{p} \in c_{p}^{k}(v)$ and $\left[Y_{p}, c_{-1}^{k}(v)\right]=0$, then $Y_{p}=0(-k \leqq p \leqq-2)$.

(iii) $E$ and $Q^{k}(v)$ are abelian subalgebras of $c^{k}(v)$ such that

$$
c_{-1}^{k}(v)=E \oplus Q^{k}(v)(\text { direct sum }),
$$




$$
Q^{k}(v)=\left\{X \in c_{-1}^{k}(v) \mid\left[X, c_{p}^{k}(v)\right]=0\right\} \quad \text { for }-k \leqq p \leqq-2 .
$$

(iv) If $X \in Q^{k}(v)$ and $\left[X, c_{-1}^{k}(v)\right]=0$, then $X=0$.

(v) $\tilde{\alpha}_{p}\left(Y_{p}, X_{1}, \cdots, X_{p+k+1}\right)(-k \leqq p \leqq-2)$ is symmetric with respect to $X_{1}, \cdots, X_{p+k+1}$, i.e., $\tilde{\alpha}_{p}$ defines the bilinear map $\alpha_{p}: c_{p}^{k}(v) \times S^{p+k+1}\left(c_{-1}^{k}(v)\right) \rightarrow$ $c_{-(k+1)}^{k}(v)$. Furthermore $\alpha_{p}$ induces a unique bilinear map $\beta_{p}: c_{p}^{k}(v) \times S^{p+k+1}(V(v))$ $c_{-(k+1)}^{k}(v)$ such that $\alpha_{p}=\beta_{p} \cdot \mu_{p}$, where $\mu_{p}=i d_{c_{p}^{k}(v)} \times \otimes{ }^{p+k+1} \kappa$.

(vi) $\tilde{\alpha}_{-1}$ defines the bilinear map $\alpha_{-1}: Q^{k}(v) \times S^{k}\left(c_{-1}^{k}(v)\right) \rightarrow c_{-(k+1)}^{k}(v)$. Furthermore, $\alpha_{-1}$ induces a unique bilinear map $\beta_{-1}: Q^{k}(v) \times S^{k}(V(v)) \rightarrow c_{-1}^{k}(v)$ such that $\alpha_{-1}=\beta_{-1} \cdot \mu_{-1}$, where $\mu_{-1}=i d_{Q^{k}(v)} \times \otimes^{k} \kappa$.

Proof. (i ) The first assertion is clear by the definition of $c^{k}(v)$. The second assertion follows from $\partial^{(r)} C^{k}=\partial^{r} C^{k}$ for $r=1, \cdots, k$ (cf. Lemma 5.6 (ii)), or from the fact that $c^{k}(v)$ is isomorphic with $\mathcal{C}^{k}(n)$.

(ii) Since $c(v)$ is isomorphic with $\mathcal{S}^{k}(n)$ (Lemma 3.4 (ii)), the assertion follows from the definition of the bracket operation of $\varsigma^{k}(n)$. (The more intrinsic proof is given in that of Lemma 5.6 (iii)).

(iii) Since $E$ is an integral element of $\left(J^{k}(\mathfrak{S}), C^{k}\right), E$ is an abelian subalgebra of $c^{k}(v)$. And, since $Q^{k}$ is completely integrable, $Q^{k}(v)$ is also an abelian subalgebra of $c^{k}(v)$. Furthermore it is easy to see that the last assertion is equivalent to $Q^{k}=C^{k} \cap C h\left(C_{r}^{k}\right)$ for $r=1, \cdots, k-1$ (cf. The proof of Lemma 5.6 (vi)), which follows from Proposition 2.5 (3).

(iv) The assertion is equivalent to $C h\left(C^{k}\right)(v)=\{0\}$, which follows from the proof of Proposition 2.5 (2).

(v) From $\left[c_{p}^{k}(v), c_{q}^{k}(v)\right]=\{0\}$ for $p, q<-1$, we get

$$
\text { [[ } \left.\left.Y_{q}, X_{1}\right], X_{2}\right]=\left[\left[Y_{q}, X_{2}\right], X_{1}\right]
$$

for $\left[Y_{q} \in \mathfrak{c}_{p}^{k}(v)(q<-1)\right.$ and $X_{1}, X_{2} \in \mathfrak{c}_{-1}^{k}(v)$. Hence we see that $\hat{\alpha}_{p}\left(Y_{p}, X_{1}, \cdots\right.$, $\left.X_{p+k+1}\right)$ is symmetric with respect to $X_{1}, \cdots, X_{p+k+1}$. Furthermore, from $\left[c_{p}^{k}(v), Q^{k}(v)\right]=\{0\}$ for $p<-1$, we see that

$$
\hat{\alpha}_{p}\left(Y_{p}, X_{1}, \cdots, X_{p+k+1}\right)=0 \quad \text { if } X_{i} \in Q^{k}(v) \text { for some } i \text {. }
$$

Hence the last assertion follows immediately.

Similarly, (vi) follows from $\left[\mathfrak{c}_{p}^{k}(v), \mathfrak{c}_{q}^{k}(v)\right]=\{0\}$ and $\left[\mathfrak{c}_{p}^{k}(v), Q^{k}(v)\right]=\{0\}$ for $p, q$ $<-1$. q.e.d.

Let $\tau_{p}(v)(-k \leqq p \leqq-2)$ (resp. $\left.\tau_{-1}(v)\right)$ be the linear map of $c_{p}^{k}(v)\left(\right.$ resp. $\left.Q^{k}(v)\right)$ into $c_{-(k+1)}^{k}(v) \otimes S^{p+k+1}\left(V(v)^{*}\right)\left(\right.$ resp. $\left.c_{-(k+1)}^{k}(v) \otimes S^{k}\left(V(v)^{*}\right)\right)$ defined by

$$
\tau_{p}(v)\left(Y_{p}\right)\left(\tilde{X}_{1} \bigcirc \ldots \bigcirc \tilde{X}_{p+k+1}\right)=\beta_{p}\left(Y_{p}, \tilde{X}_{1} \bigcirc \ldots \bigcirc \tilde{X}_{p+k+1}\right)(-k \leqq p \leqq-1),
$$

for $Y_{-1} \in Q^{k}(v), Y_{p} \in \mathfrak{c}_{p}^{k}(v) \quad(p \leqq-2)$ and $\tilde{X}_{i} \in V(v) \quad(i=1, \cdots, p+k+1)$. Let $\left(\rho_{k}^{k+1}\right)^{-1}(v)$ denote the fibre of $\rho_{k}^{k+1} ; J^{k+1}\left(\right.$ (5) $\rightarrow J^{k}($ (5) over $v$ and let $w=E$ be any 
point of $\left(\rho_{k}^{k+1}\right)^{-1}(v)$. We denote by $\phi_{w}$ the linear map of $c^{k}(v)$ into $\mathcal{S}^{k}(V(v)$, $\left.c_{-(k+1)}^{k}(v)\right)$ defined by

$$
\begin{aligned}
& \left.\phi_{w}\right|_{W_{c}}=i d_{W_{c}},\left.\quad \phi_{w}\right|_{E}=\left.\kappa\right|_{E}, \\
& \left.\phi_{w}\right|_{Q^{k}(v)}=\tau_{-1}(v),\left.\quad \phi_{w}\right|_{c_{p}^{k}(v)} ^{k}=\tau_{p}(v)(-k \leqq p \leqq-2),
\end{aligned}
$$

where $W_{c}=c_{-(k+1)}^{k}(v)$. Furthermore, let $S^{k+1}(\mathfrak{S})$ denote the vector bundle over $J^{k}($ (ङ) defined by

$$
S^{k+1}(\mathfrak{C})=\bigcup_{v \in J k(\mathfrak{S})} c_{-(k+1)}^{k}(v) \otimes S^{k+1}\left(V(v)^{*}\right)(k \geqq 2) .
$$

Then we have

Proposition 3.9. Let $v$ be any point of $J^{k}(\mathcal{S})$ and $\tau_{p}(v), \phi_{w}$ be as above $(-k \leqq p \leqq-1)$.

(1) $\tau_{-1}(v)$ is a linear isomorphism of $Q^{k}(v)$ onto $c_{-(k+1)}^{k}(v) \otimes S^{k}\left(V(v)^{*}\right)$. And $\tau_{p}(v)$ is a linear isomorphism of $c_{p}^{k}(v)$ onto $c_{-(k+1)}^{k}(v) \otimes S^{p+k+1}\left(V(v)^{*}\right)$ for $p=-2, \cdots,-k$.

(2) Let $w$ be any point of $\left(\rho_{k}^{k+1}\right)^{-1}(v)$. Then $\phi_{w}$ is a graded Lie algebra isomorphism of $\mathrm{c}^{k}(v)$ onto $\mathfrak{C}^{k}\left(V(v), \mathrm{c}_{-(k+1)}^{k}(v)\right)$.

(3) The vector group $\mathfrak{c}_{-(k+1)}^{k}(v) \otimes S^{k+1}\left(V(v)^{*}\right)$ canonically acts simply transitively on $\left(\rho_{k}^{k+1}\right)^{-1}(v)$, i.e., $\left(\rho_{k}^{k+1}\right)^{-1}(v)$ is an affine space modeled on $c_{-(k+1)}^{k}(v)$ $\otimes S^{k+1}\left(V(v)^{*}\right)$. Hence $J^{k+1}(\mathfrak{S})$ is an affine bundle over $J^{k}(\mathfrak{S})$ modeled on $S^{k+1}(\mathfrak{S})$ for $k \geqq 2$.

Proof. (1) We write $\tau_{p}=\tau_{p}(v)$. If $\tau_{p}\left(Y_{p}\right)=0$, we have

$$
\tilde{\alpha}_{p}\left(Y_{p}, X_{1}, \cdots, X_{p+k+1}\right)=\left[\left[\cdots\left[Y_{p}, X_{1}\right], \cdots\right], X_{p+k+1}\right]=0
$$

for any $X_{i} \in \mathfrak{c}_{-1}^{k}(v)(i=1, \cdots, p+k+1)$. Then, by using (ii) and (iv) of Lemma 3.8 repeatedly, we get $Y_{p}=0$. Hence $\tau_{p}$ is injective for $p=-1, \cdots,-k$. Therefore, by comparing the dimensions, we obtain the assertions.

(2) Since $\left.\kappa\right|_{E}$ is a linear isomorphism of $E=w$ onto $V(v)$ and $E, Q^{k}(v)$ are abelian subalgebras, the assertion follows immediately from $\left[c_{p}^{k}(v), c_{q}^{k}(v)\right]$ $=\left[c_{p}^{k}(v), Q^{k}(v)\right]=\{0\}$ for $p, q<-1$ and the definition of $\tau_{p}(v)(-k \leqq p \leqq-1)$.

(3) By Proposition $3.7(1), c_{-(k+1)}^{k}(v) \otimes S^{k+1}\left(V(v)^{*}\right)$ is canonically isomorphic with $N=N^{k}\left(V(v), c_{-(k+1)}^{k}(v)\right)$. Hence it suffices to show that $N$ acts simply transitively on $\left(\rho_{k}^{k+1}\right)^{-1}(v)$. Let $w$ be any element of $\left(\rho_{k}^{k+1}\right)^{-1}(v)$ and let $A_{\rho}$ be any element of $N$. Set $w^{\prime}=\left(A_{\rho} \cdot \phi_{w}\right)^{-1}(V(v))$, where $\phi_{w}$ is as in (2). Then $w^{\prime} \in\left(\rho_{k}^{k+1}\right)^{-1}(v)$ and it follows easily that $A_{\rho} \cdot \phi_{w}=\phi_{w^{\prime}}$. Since $\phi_{w}=\phi_{w^{\prime}}$ if and only if $w=w^{\prime}$, we have thus defined the action of $N$ on $\left(\rho_{k}^{k+1}\right)^{-1}(v)$. Clearly $\phi_{w^{\prime}} \cdot\left(\phi_{w}\right)^{-1} \in N$ for $w, w^{\prime} \in\left(\rho_{k}^{k+1}\right)^{-1}(v)$. Hence $N$ acts simply transitively on $\left(\rho_{k}^{k+1}\right)^{-1}(v)$. Therefore it follows that $J^{k+1}(\mathfrak{S})$ is an affine bundle over $J^{k}(\mathfrak{S})$ modeled on $S^{k+1}$ (ङ) for $k \geqq 2$ (cf. $[9, \S 5]$ ). 
$\tau_{-1}(v): Q^{k}(v) \rightarrow c_{-(k+1)}^{k}(v) \otimes S^{k}\left(V(v)^{*}\right)$ is called the fundamental identification at $v \in J^{k}(5)$. One should note that $\tau_{-1}(v)$ is determined solely by the structure of $c^{k}(v)$. Hence the fundamental identification has the invariant meaning under contact transformations.

REMARK 3.10. Let $(M, N, p)$ be a fibred manifold of fibre dimension 1 , and let $J^{k}(M, N, p)$ be the bundle of $k$-jets of $(M, N, p) . \quad J^{k}(M, N, p)$ is canonically imbedded into $J^{k}(\mathfrak{S})$ as an open submanifold, where c denotes the contact manifold $\left(J^{1}(M, N, p), C^{1}\right)$ (cf. Remark 3.3). Let $\bar{C}^{k}$ denote the canonical system on $J^{k}(M, N, p)$. Let $z$ be any point of $J^{k}(M, N, p)$ and $c^{k}(z)$ be the graded algebra of $\left(J^{k}(M, N, p), \bar{C}^{k}\right)$ at $z$. Set $Q^{k}(z)=\operatorname{Ker}\left(p_{k-1}^{k}\right)_{*}$, $V(z)=\bar{C}^{k}(z) / Q^{k}(z), Q_{y}(M)=\operatorname{Ker}\left(p_{*}\right)_{y}, \quad y=p_{0}^{k}(z)$ and $x=p_{-1}^{k}(z)$. Then $\left(p_{-1}^{k}\right)_{*}$ : $T_{z}\left(J^{k}(M, N, p)\right) \rightarrow T_{x}(N)$ induces a linear isomorphism $\hat{p}$ of $V(z)$ onto $T_{x}(N)$. Furthermore $p_{*}: T_{y}(M) \rightarrow T_{x}(N)$ induces a linear isomorphism $q_{1}$ of $Q_{y}(M)$ onto $T_{y}(M) / T_{x}(N)$ and $\left(p_{0}^{k}\right)_{*}: T_{z}\left(J^{k}(M, N, p)\right) \rightarrow T_{y}(M)$ induces a linear isomorphism $q_{2}$ of $c_{-(k+1)}^{k}(z)$ onto $T_{y}(M) / T_{x}(N)$. Hence $\hat{q}=q_{1}^{-1} \cdot q_{2}$ is a linear isomorphism of $c_{-(k+1)}^{k}(z)$ onto $Q_{y}(M)$. Therefore $c_{-(k+1)}^{k}(z) \otimes S^{k}\left(V(z)^{*}\right)$ is identified with $Q_{y}(M) \otimes S^{k}\left(T_{x}^{*}(N)\right)$ via $\gamma=\hat{q} \times\left(\otimes{ }^{k} \hat{p}^{*}\right)^{-1}$. Then it is easy to see that the identification $\gamma \cdot \tau_{-1}(z): Q^{k}(z) \rightarrow Q_{y}(M) \otimes S^{k}\left(T_{x}^{*}(N)\right)$ coincides with the fundamental identification in the sense of M. Kuranishi [15] (cf. Proposition 2.2 [15] or Proposition 5.2 [9]).

\section{§4. Submanifolds of $\left(J^{k}(\mathcal{S}), C^{k}\right)$}

4.1. Submanifolds of $\left(J^{k}(\mathfrak{S}), C^{k}\right)$. Let $R$ be a submanifold of $\left(J^{k}(\mathfrak{S}), C^{k}\right)$ and let $\iota$ be the inclusion of $R$ into $J^{k}(\varsigma)$. We set $\bar{\rho}_{r}=\rho_{r}^{k} \cdot \iota(1 \leqq r \leqq k)$, hence $\bar{\rho}_{k}=\iota$. We impose on $R$ the following two conditions:

(c.1) $D^{r}=\left(\bar{\rho}_{r}\right)_{*}^{-1}\left(C^{r}\right)$ is a differential system on $R$ for $r=1, \cdots, k$, i.e., $\operatorname{dim} D^{r}(x)$ is constant on $R(1 \leqq r \leqq k)$, where $D^{r}(x)=\left(\bar{\rho}_{r}\right)_{*}^{-1}\left(C^{r}\left(\bar{\rho}_{r}(x)\right)\right), x \in R$ and $C^{r}$ is the canonical system on $J^{r}(\mathbb{S})$.

(c.2) $\left(R, J^{1}(\mathcal{S}), \bar{\rho}_{1}\right)$ is a fibred manifold, i.e., $\bar{\rho}_{1}$ is a submersion of $R$ onto $J^{1}(\mathfrak{C})$.

The condition (c.1) is a generic one and $\left(D^{1}, \cdots, D^{k}\right)$ is called the contact system of order $k$ associated with $R$. $R$ being considered as a system of partial differential equations of order $k$, the condition (c.2) means that $R$ has no equation of first order.

Let $x$ be any point of $R$. Let $F^{r}(x)(r=1, \cdots, k-1)$ denote the kernel of $\left(\bar{\rho}_{r}\right)_{*}: T_{x}(R) \rightarrow T_{p r(x)}\left(J^{r}(\widetilde{c})\right)$, i.e., $F^{r}(x)=\iota_{*}^{-1}\left(Q_{r}^{k}(\iota(x))\right)$. Then, by Proposition 2.5 $(2), F^{r}(x)$ is a subspace of $D^{r+1}(x)$ of codimension at most $n$ for $r=1, \cdots, k-1$. First we have 
LEMma 4.1. Let $R$ be a submanifold of $J^{k}(\mathfrak{S})$ satisfying (c.1). Let $x$ be any point of $R$.

(i) The derived sheaf $\partial \mathscr{D}^{r+1}$ of $D^{r+1}$ is a subsheaf of the sheaf $\mathscr{D}^{r}$ of $D^{r}$ for $r=1, \cdots, k-1$.

(ii) $F^{r}(x)=F^{1}(x) \cap D^{r+1}(x) \quad$ for $r=2, \cdots, k-1$.

(iii) $F^{1}(x) \subset C h\left(D^{1}\right)(x)$.

(iv) $F^{r}(x) \subset F^{r-1}(x) \cap C h\left(D^{r}\right)(x) \quad$ for $r=2, \cdots, k-1$.

Proof. Let $\left(x_{i}, z, p_{I}\right)\left(i=1, \cdots, n, I \in \Sigma_{k}\right)$ be a canonical coordinate system on a neighborhood $U_{k}$ of $\iota(x) \in J^{k}(\mathfrak{C})$, which is subordinate to $(x, z, p)$ (Lemma 2.3). We set $x_{i}^{*}=x_{i} \cdot \iota, z^{*}=z \cdot \iota$ and $p_{I}^{*}=p_{I} \cdot \iota \quad\left(i=1, \cdots, n, I \in \Sigma_{k}\right)$. Then we have $(r=1, \cdots, k-1)$

$$
F^{r}(x)=\left\{X \in T_{x}(R) \mid \mathrm{d} x_{i}^{*}(X)=\mathrm{d} z^{*}(X)=\mathrm{d} p_{I}^{*}(X)=0\left(1 \leqq i \leqq n, I \in \Sigma_{r}\right)\right\} .
$$

Furthermore, by Lemma 2.3, we see that $D^{r+1}$ is defined on $\iota^{-1}\left(U_{k}\right)$ by the following 1-forms:

$$
\begin{aligned}
& \pi^{*}=\mathrm{d} z^{*}-\sum_{i=1}^{n} p_{i}^{*} \mathrm{~d} x_{i}^{*}, \\
& \pi_{I}^{*}=\mathrm{d} p_{I}^{*}-\sum_{j=1}^{n} p_{I, j}^{*} \mathrm{~d} x_{j}^{*}\left(I \in \Sigma_{r}\right) .
\end{aligned}
$$

Hence we get (ii). From $\mathrm{d} \pi^{*} \equiv 0\left(\bmod \left(F^{1}(x)\right)^{\perp}\right)$, we get (iii). Furthermore, since $\mathrm{d} \pi^{*} \equiv \mathrm{d} \pi_{I}^{*} \equiv 0\left(\bmod \left(D^{r+1}(x)\right)^{\perp}\right)$ for $I \in \Sigma_{r-1}$, it follows from Lemma 1.1 that $\pi^{*}, \pi_{I}^{*} \in\left(\partial D^{r+1}(x)\right)^{\perp}$ for $I \in \Sigma_{r-1}$, i.e., $\partial D^{r+1}(x) \subset D^{r}(x)$ at each $x \in R$. Hence $\partial \mathscr{D}^{r+1} \subset \mathscr{D}^{r}$. Thus we get (i). By Lemma 1.2, we have

$$
C h\left(D^{r+1}\right)(x)=\left\{X \in D^{r+1}(x) \mid X \smile\left(\mathrm{d} \pi_{I}^{*}\right)_{x} \equiv 0\left(\bmod \left(D^{r+1}(x)\right)^{\perp}\right) \text { for } I \in S_{r}\right\} .
$$

Hence, from $\mathrm{d} \pi_{I}^{*}=\sum_{j=1}^{n} \mathrm{~d} x_{j}^{*} \wedge \mathrm{d} p_{I, j}^{*}$ and $F^{r}(x) \subset D^{r+1}(x)$, we get

$$
F^{r}(x) \cap C h\left(D^{r+1}\right)(x)=\left\{X \in F^{r}(x) \mid \sum_{j=1}^{n} \mathrm{~d} p_{I, j}^{*}(X) \mathrm{d} x_{j}^{*} \equiv 0\left(\bmod \left(D^{r+1}(x)\right)^{\perp}\right) \text { for } I \in S_{r}\right\} .
$$

Therefore $F^{r+1}(x)$ is contained in $F^{r}(x) \cap C h\left(D^{r+1}\right)(x)$.

q.e.d.

LEMMA 4.2. Let $R$ be a submanifold of $J^{k}(5)$ satisfying (c.2).

(i ) $D^{1}$ and $D^{2}$ are differential systems of codimension 1 and $n+1$ respectively.

(ii) $F^{1}$ is a subbundle of $D^{2}$ of codimension $n$ and coincides with $C h\left(D^{1}\right)$.

Proof. We let notations be the same as in the proof of Lemma 4.1. Since $(x, z, p)$ is a canonical coordinate system of $J^{1}(\mathcal{E})$ on $\rho_{1}^{k}\left(U_{k}\right)$, the condition (c.2) holds if and only if $\mathrm{d} x_{i}^{*}, \mathrm{~d} p_{i}^{*}$ and $\mathrm{d} z^{*}(i=1, \cdots, n)$ are linearly independent everywhere on $\iota^{-1}\left(U_{k}\right)$. $D^{1}$ is defined on $\iota^{-1}\left(U_{k}\right)$ by $\pi^{*}$ and $D^{2}$ is 
defined by $\pi_{1}^{*}, \cdots, \pi_{n}^{*}$ and $\pi^{*}$. Hence we get (i). Furthermore, by Lemma 1.2 , it is easy to see that $C h\left(D^{1}\right)$ is defined on $c^{-1}\left(U_{k}\right)$ by $\mathrm{d} x_{i}^{*}, \mathrm{~d} p_{i}^{*}$ and $\mathrm{d} z^{*}$ $(i=1, \cdots, n)$. Hence we obtain $F^{1}=C h\left(D^{1}\right)$.

Now we impose further on $R$ the following condition:

q.e.d.

(c.3) $F^{k-1}$ is a subbundle of $D^{k}$ of codimension $n$.

As for the condition (c.3), we note

LemMA 4.3. Let $R$ be a submanifold of $J^{k}(\mathfrak{S})$ satisfying (c.1). Then

(i) The condition (c.3) holds if, for each point $x$ of $R$, there exists a point $w$ of $J^{k+1}(\mathfrak{S})$ such that $\rho_{k}^{k+1}(w)=x$ and $w$ is a subspace of $T_{x}(R)$, i.e., if $\rho^{(1)} ; R^{(1)}$ $\rightarrow R$ is onto, where $R^{(1)}$ is the first prolongation of $R$.

(ii) If (c.3) holds, then $F^{r}$ is a subbundle of $D^{r+1}$ of codimension $n$ for $r=1, \cdots, k-1$ and satisfies

$$
F^{r}(x)=F^{r-1}(x) \cap C h\left(D^{r}\right)(x), F^{k-1}(x) \cap C h\left(D^{k}\right)(x)=\{0\} \text { at each } x \in R .
$$

Proof. Notations being the same as in the proof of Lemma 4.1, we see that $D^{r}$ is defined on $\iota^{-1}\left(U_{k}\right)$ by $\pi^{*}$ and $\pi_{I}^{*}\left(I \in \Sigma_{r-1}\right)$ and that $F^{r-1}$ is defined on $\iota^{-1}\left(U_{k}\right)$ by $\mathrm{d} x_{1}^{*}, \cdots, \mathrm{d} x_{n}^{*}, \pi^{*}$ and $\pi_{I}^{*}\left(I \in \Sigma_{r-1}\right)$. It follows that $F^{k-1}(x)$ is a subspace of $D^{k}(x)$ of codimension at most $n$ at each $x \in R$. Hence (i) follows immediately. Furthermore, if (c.3) holds, we see that $\mathrm{d} x_{1}^{*}, \cdots, \mathrm{d} x_{n}^{*}$ are linearly independent modulo $\left(D^{k}(x)\right)^{\perp}$ at each point $x$ of $\iota^{-1}\left(U_{k}\right)$. By $D^{k}(x) \subset$ $D^{r}(x)$ for $r=1, \cdots, k, \mathrm{~d} x_{1}^{*}, \cdots, \mathrm{d} x_{n}^{*}$ are linearly independent modulo $\left(D^{r}(x)\right)^{\perp}$. Hence $F^{r}$ is a subbundle of $D^{r+1}$ of codimension $n$ for $r=1, \cdots, k-1$. Furthermore, from the proof of Lemma 4.2 (ii), we get $F^{r}(x) \cap C h\left(D^{r+1}\right)=$ $F^{r+1}(x)$ and

$$
F^{k-1}(x) \cap C h\left(D^{k}\right)(x)=\left\{X \in F^{k-1}(x) \mid \mathrm{d} p_{I}^{*}(X)=0 \text { for } I \in S_{k}\right\}=\{0\} .
$$

Thus we obtain (ii).

q.e.d.

Summarizing the discussion above, we obtain

Proposition 4.4. Let $R$ be a submanifold of $J^{k}(\Subset)$ satisfying (c.1), (c.2) and (c.3). Then

(1) $D^{1}$ and $D^{2}$ are differential systems of codimension 1 and $n+1$ respectively.

(2) The derived sheaf $\partial \mathscr{D}^{r+1}$ of $D^{r+1}$ is a subsheaf of the sheaf $\mathscr{D}^{r}$ of $D^{r}$ for $r=1, \cdots, k-1$.

(3) $F^{r}=\operatorname{Ker}\left(\bar{\rho}_{r}\right)_{*}$ is a subbundle of $D^{r+1}$ of codimension $n$ for $r=1, \cdots$, $k-1$ and satisfies

$$
F^{r}(x)=C h\left(D^{1}\right)(x) \cap \cdots \cap C h\left(D^{r}\right)(x) \quad \text { at each } x \in R .
$$


(4) $F^{k-1}(x) \cap C h\left(D^{k}\right)(x)=\{0\}$ at each $x \in R$.

(5) If $k \geqq 3, F^{r}$ coincides with $F^{r-1} \cap D^{r+1}$ for $r=2, \cdots, k-1$, i.e., $F^{r}(x)$ $=F^{r-1}(x) \cap D^{r+1}(x)$ at each $x \in R$. In particular $F^{k-1}=F^{1} \cap D^{k}$.

REMARK 4.5. (1) One should note that, in case $k=2$, the conditions (c.1) and (c.3) follow from (c.2) (Lemma 4.2). More generally we consider the following condition for $R$ :

$\left(c^{\prime} .2\right) \quad\left(R, J^{k-1}\left(\varsigma^{5}\right), \bar{\rho}_{k-1}\right)$ is a fibred manifold.

As in Lemma 4.2, it is easy to see that ( $\left.c^{\prime} .2\right)$ implies (c.1) and (c.3). A submanifold of $J^{k}(\mathfrak{S})$ satisfying $\left(\mathrm{c}^{\prime} .2\right)$ is called a principal submanifold. For a principal submanifold $R$ of $J^{k}(\mathfrak{S})(k \geqq 3)$, it follows easily that $\partial D^{r+1}=D^{r}$ for $r=1, \cdots, k-2$ (cf. Proposition $5.11(1)$ ). Hence, by Lemma 1.3, we also obtain $F^{r}=C h\left(D^{r}\right)$ for $r=1, \cdots, k-1$.

(2) Let $R$ be a submanifold of $J^{k}$ (ङ) satisfying (c.1), (c.2) and (c.3). Since $F^{k}=\operatorname{Ker}\left(\bar{\rho}_{r}\right)_{*}$ is a differential system on $R$ (Lemma 4.3 (ii)), $\bar{\rho}_{r}$ is a map of $R$ into $J^{r}(\mathfrak{S})$ of constant rank for $r=1, \cdots, k-1$. Hence $\bar{\rho}_{r}(R)$ is locally a submanifold of $J^{r}(\mathfrak{S})$. More precisely, let $x_{0}$ by any point of $R$. Then there exists a neighborhood $U$ of $x_{0}$ such that $\bar{\rho}_{r}(U)$ is a submanifold of $J^{k}(\widetilde{E})$ for $r=1, \cdots, k-1$. It is easy to see that $\bar{\rho}_{r}(U)$ satisfies the conditions (c.1) and (c.2). Furthermore, for $x \in U, \bar{\rho}_{r+1}(x) \in J^{r+1}(\mathfrak{S})$ is a subspace of $T_{p r(x)}\left(\bar{\rho}_{r}(U)\right)$. Hence $\bar{\rho}_{r}(U)$ also satisfies (c.3) by Lemma 4.3 (i). Let $\bar{D}^{r}$ be the restriction of $C^{r}$ to $\bar{\rho}_{r}(U)$. Then we note that $\left(\bar{\rho}_{r}(U), \bar{D}^{r}\right)$ has a trivial Cauchy-Cartan characteristic system if and only if $F^{r}=C h\left(D^{r}\right)$, i.e., if and only if $C h\left(D^{r}\right) \subset$ $C h\left(D^{s}\right)$ for every $s<r$.

4.2. Involutive submanifolds. Let $R$ be a submanifold of $J^{k}(\mathfrak{C})(k \geqq 2)$. As is well known, the first prolongation $R^{(1)}$ of $R$ is, by definition (cf. Definition 4.1 [15] or Definition 7.1 [9]), the set of points of $J^{k+1}(5)$ which are contact elements to $R$, i.e.,

$$
R^{(1)}=J(R, n) \cap J^{k+1}(\Im) \subset J\left(J^{k}(\Im), n\right) .
$$

Let $x$ be any point of $R$. Then the kernel $F^{k-1}(x)$ of $\left(\bar{\rho}_{k-1}\right)_{*}: T_{x}(R) \rightarrow T_{y}\left(J^{k-1}(\mathfrak{S})\right)$, $y=\bar{\rho}_{k-1}(x)$, is a subspace of $Q^{k}(x)$. Hence $F^{k-1}(x)$ can be considered as a subspace of $c_{-(k+1)}^{k}(x) \otimes S^{k}\left(V(x)^{*}\right)$ via the fundamental identification $\tau_{-1}(x): Q^{k}(x)$ $\rightarrow c_{-(k+1)}^{k}(x) \otimes S^{k}\left(V(x)^{*}\right)$ (Proposition 3.8). Therefore it is meaningful to say that $F^{k-1}(x)$ is an involutive subspace of $c_{-(k+1)}^{k}(x) \otimes S^{k}\left(V(x)^{*}\right) \subset\left(c_{-(k+1)}^{k}(x) \otimes\right.$ $\left.S^{k-1}\left(V(x)^{*}\right)\right) \otimes V(x)^{*}$ as in the usual way (Definition 6.1 [15]). Let $\rho^{(1)}$ denote the restriction of $\rho_{k}^{k+1}$ to $R^{(1)}$.

Definition 4.6 (cf. Definition 7.1 [15]). Let $R$ be a submanifold of $J^{k}(\sqrt{5})$ 
$(k \geqq 2)$. $\quad R$ is called an involutive submanifold if and only if the following conditions are satisfied: $x \in R$.

(1) $F^{k-1}(x)$ is an involutive subspace of $c_{-(k+1)}^{k}(v) \otimes S^{k}\left(V(x)^{*}\right)$ at each

(2) $R^{(1)}$ is a submanifold of $J^{k+1}(5)$ such that $\rho^{(1)}$ is a submersion of $R^{(1)}$ onto $R$, i.e., $\left(R^{(1)}, R, \rho^{(1)}\right)$ is a fibred manifold.

For an involutive submanifold, we note

LEMMA 4.7. Let $R$ be an involutive submanifold of $J^{k}(\mathfrak{S})(k \geqq 2)$.

(i ) $D^{k}$ is a differential system on $R$ and $F^{k-1}$ is a subbundle of $D^{k}$ of codimension $n$.

(ii) $D^{r+1}$ is a differential system on $R$ if and only if $\bar{\rho}_{r}$ is a map of constant rank for $r=1, \cdots, k-2$.

Proof. It is well known that if $R$ is involutive, then $\operatorname{dim} F^{k-1}(x)$ is constant on $R$, i.e., $F^{k-1}$ is a differential system on $R$ (cf. Proposition 7.1 [15]). Since $\rho^{(1)} ; R^{(1)} \rightarrow R$ is onto, it is easy to see, as in the proof of Lemma 4.3, that $F^{k-1}(x)$ is a subspace of $D^{k}(x)$ of codimension $n$ at each $x \in R$. Hence we get (i). Furthermore we see that $F^{r}(x)$ is a subspace of $D^{r+1}(x)$ of codimension $n$ at each $x \in R(r=1, \cdots, k-2)$. Hence $D^{r+1}$ is a differential system on $R$ if and only if $F^{r}=\operatorname{Ker}\left(\bar{\rho}_{r}\right)_{*}$ is a differential system on $R$. Thus we get (ii).

q.e.d.

Let $R$ be an involutive submanifold of $J^{k}(\mathfrak{5})(k \geqq 2)$. Then, as is well known, each $v \in R^{(1)}$ is an ordinary integral element of $\left(R, D^{k}\right)$ (Appendix [15]). Hence, in the real analytic category, the involutiveness of $R$ guarantees, at each $x \in R$, the existence of an $n$-dimensional integral manifold $N$ of $\left(R, D^{k}\right)$ passing through $x$ such that $T_{y}(N) \cap F^{k-1}(y)=\{0\}$ for each $y \in N$.

Now we restate the involutiveness of a submanifold $R$ of $J^{k}$ (ङ) in terms of the graded subalgebras associated with $R$. By Proposition 2.5 (1), the canonical system $C^{k}$ is a regular differential system on $J^{k}(\mathbb{C})$ such that $T\left(J^{k}(\mathbb{C})\right)=\partial^{(k)} C^{k}$. Let $R$ be a submanifold of $J^{k}(\mathfrak{C})$ satisfying (c.1). Then we can consider the graded subalgebra $\mathfrak{r}(x)$ of $c^{k}(x)$ associated with $R$ at each $x \in R$, where $\mathfrak{c}^{k}(x)=\mathfrak{c}^{k}(\iota(x))$ is the graded algebra of $\left(J^{k}(\mathfrak{S}), C^{k}\right)$ at $\iota(x) \in J^{k}(\mathfrak{S})$ (cf. 1.3 of $\S 1$ ). By Proposition 3.6, $c^{k}(x)$ is isomorphic with the contact algebra $\mathfrak{C}^{k}(n)$ of $k$-th order of degree $n$. In view of Proposition 3.7, we first give the following definition.

Definition 4.8. A graded subalgebra $\mathfrak{g}=\sum_{p<0} \mathfrak{g}_{p}$ of $\mathfrak{C}^{k}(n)$ is called $i n$ volutive if and only if the following conditions are satisfied:

(1) $\mathfrak{g}_{-1}$ contains an $n$-dimensional abelian subalgebra $V_{0}$ such that $\mathfrak{z}_{-1}$ $=V_{0} \oplus \mathfrak{g}_{*}($ direct sum $)$, where $\mathfrak{F}_{*}=\mathfrak{g}_{-1} \cap \mathfrak{F}(V, W)$ ).

(2) $\mathfrak{g}_{*}$ is an involutive subspace of $\mathfrak{\xi}(V, W)=W \otimes S^{k}\left(V^{*}\right)$. 
Let $\nu$ be an isomorphism of $c^{k}(x)$ onto $\mathfrak{C}^{k}(n)$. We say that the graded subalgebra $r(x)$ of $c^{k}(x)$ is involutive if $\nu(r(x))=\xi$ is an involutive subalgebra of $\mathfrak{C}^{k}(n)$. By Proposition 3.7, this definition is independent of the choice of $\nu$. One should note that $\mathfrak{r}_{-1}(x) \cap Q^{k}(x)=F^{k-1}(x)$ and $\nu\left(F^{k-1}(x)\right)=\mathfrak{Z}_{*}$. By the fundamental identification $\tau_{-1}(x), \mathfrak{r}_{*}(x)=F^{k-1}(x)$ is identified with the subspace $\overline{\mathfrak{x}}_{*}(x)=\tau_{-1}(x)\left(F^{k-1}(x)\right)$ of $c_{-(k+1)}^{k}(x) \otimes S^{k}\left(V(x)^{*}\right)$. These being remarked, we define the first prolongation $p\left(\mathfrak{r}_{*}(x)\right)$ of $\mathfrak{r}_{*}(x)$ by $([15, \mathrm{p} .18])$

$$
p\left(\mathfrak{x}_{*}(x)\right)=\overline{\mathfrak{x}}_{*}(x) \otimes V(x)^{*} \cap \mathfrak{c}_{-(k+1)}^{k}(x) \otimes S^{k+1}\left(V(x)^{*}\right) .
$$

$p\left(\mathfrak{r}_{*}(x)\right)$ is, by definition, a subspace of $c_{-(k+1)}^{k}(x) \otimes S^{k+1}\left(V(x)^{*}\right) . \quad$ Then we have

Proposition 4.9. Let $R$ be a submanifold of $J^{k(5)}$ satisfying (c.1). Then $R$ is an involutive submanifold if and only if the following conditions are satisfied:

(1) $\mathfrak{r}(x)$ is an involutive subalgebra of $c^{k}(x)$ at each $x \in R$.

(2) $\operatorname{dim} p\left(\mathfrak{r}_{*}(x)\right)$ is constant on $R$.

Proof. The following fact is well known (cf. Lemma 9.1 [15] or Proposition $7.1[9]):\left(R^{(1)}, R, \rho^{(1)}\right)$ is a fibred manifold if and only if $\rho^{(1)}: R^{(1)} \rightarrow R$ is onto and $\operatorname{dim} p\left(\mathfrak{r}_{*}(x)\right)$ is constant on $R$. Then the assertion is a direct consequence of this fact.

q.e.d.

4.3. Contact equivalence of involutive submanifolds. Let $R$ be a submanifold of $J^{k}(\mathfrak{C})$ satisfying (c.1), (c.2) and (c.3). We say that $R$ is regular with respect to $\bar{\rho}_{1}$ if $\iota ; R \rightarrow J^{k}(\mathfrak{S})$ is a homeomorphism into $J^{k}(\mathfrak{S})$ and each fibre of $\bar{\rho}_{1} ; R \rightarrow J^{1}(R)$ is connected. One should note that a submanifold of $J^{k}(\mathfrak{C})$ satisfying (c.1), (c.2) and (c.3) is always locally regular with respect to $\bar{\rho}_{1}$.

THEOREM 4.10. Let $\mathbb{C}$ and $\hat{\mathbb{C}}$ be contact manifolds of dimension $2 n+1$, and let $\left(J^{k}(\mathfrak{S}), C^{k}\right)\left(\right.$ resp. $\left.\left(J^{k}(\hat{\mathbb{C}}), \hat{C}^{k}\right)\right)$ be the $k$-th order prolongation of $\mathfrak{C}$ (resp. $\hat{\mathfrak{E}})$. Let $R$ (resp. $\hat{R})$ be a submanifold of $J^{k}(\mathfrak{E})$ (resp. $J^{k}(\hat{\mathfrak{C}})$ ) satisfying (c.1), (c.2) and (c.3), which is regular with respect to $\bar{\rho}_{1}\left(\right.$ resp $\left.\hat{\rho}_{1}\right)$. And let $\left(D^{1}, \cdots, D^{k}\right)$ (resp. $\left(\hat{D}^{1}, \cdots, \hat{D}^{k}\right)$ ) be the contact system of $k$-th order associated with $R$ (resp. $\hat{R})$. Then a contact transformation $\phi$ of $\mathbb{C}$ onto $\hat{\mathbb{E}}$ such that $\mathrm{q}^{k} \phi(R)=\hat{R}$ induces an isomorphism $\psi$ of $\left(R ; D^{1}, \cdots, D^{k}\right)$ onto $\left(\hat{R} ; \hat{D}^{1}, \cdots, \hat{D}^{k}\right)$, where $\psi=\left.\mathrm{q}^{k} \phi\right|_{R}$.

Conversely an isomorphism $\psi$ of $\left(R ; D^{1}, \cdots, D^{k}\right)$ onto $\left(\hat{R} ; \hat{D}^{1}, \cdots, \hat{D}^{k}\right)$ induces a unique contact transformation $\phi$ of $\mathbb{E}$ onto $\hat{\mathbb{E}}$ such that $\hat{\iota} \cdot \psi=\mathrm{q}^{k} \phi \cdot \iota$, where $\iota($ resp. $\hat{\imath})$ is the inclusion of $R($ resp. $\hat{R})$ into $J^{k}(\mathfrak{S})\left(\right.$ resp. $\left.J^{k}(\hat{\mathbb{C}})\right)$.

Furthermore $\hat{R}=\mathrm{q}^{k} \phi(R)$ is involutive if and only if $R$ is involutive.

Proof. By Theorem 3.2, $\mathrm{q}^{k} \phi$ preserves the canonical systems. Hence 
we have $\mathrm{q}^{k} \phi\left(\partial^{(k-r)} C^{k}\right)=\partial^{(k-r)} \hat{C}^{k}$ for $r=1, \cdots, k$. Since $\mathrm{q}^{k} \phi(R)=\hat{R}$ and $\hat{R}$ is a regular submanifold of $J^{k}(\hat{\complement}), \psi=\mathrm{q}^{k} \phi \cdot \iota$ is a diffeomorphism of $R$ onto $\hat{R}$. Therefore, from $D^{r}=\iota_{*}^{-1}\left(\partial^{(k-r)} C^{k}\right)$ and $\hat{D}^{r}=\hat{\imath}_{*}^{-1}\left(\partial^{(k-r)} \hat{C}^{k}\right)$, we see that $\psi$ is an isomorphism of $\left(R ; D^{1}, \cdots, D^{k}\right)$ onto $\left(\hat{R} ; \hat{D}^{1}, \cdots, \hat{D}^{k}\right)$.

Let $\psi$ be an isomorphism of $\left(R ; D^{1}, \cdots, D^{k}\right)$ onto $\left(\hat{R} ; \hat{D}^{1}, \cdots, \hat{D}^{k}\right)$. Since $R$ is regular with respect to $\bar{\rho}_{1}$, each fibre of $\bar{\rho}_{1}: R \rightarrow J^{1}(\mathbb{E})$ is a maximal integral manifold of $C h\left(D^{1}\right)=F^{1}$. Similarly each fiber of $\hat{\bar{\rho}}_{1}: \hat{R} \rightarrow J^{1}(\hat{\mathfrak{S}})$ is a maximal integral manifold of $C h\left(\hat{D}^{1}\right)$. Hence, from $\psi_{*}\left(D^{1}\right)=\hat{D}^{1}$, it follows that $\psi$ is fibre-preserving, i.e., there exists a unique diffeomorphism $\phi$ of $J^{1}(\mathbb{E})$ onto $J^{1}(\hat{\mathbb{E}})$ such that $\hat{\bar{\rho}}_{1} \cdot \psi=\phi \cdot \bar{\rho}_{1}$. Furthermore, by $D^{1}=\left(\bar{\rho}_{1}\right)_{*}^{-1}\left(C^{1}\right)$ and $\hat{D}^{1}=\left(\hat{\bar{\rho}}_{1}\right)_{*}^{-1}\left(\hat{C}^{1}\right)$, $\phi$ is a contact transformation, i.e., $\phi_{*}\left(C^{1}\right)=\hat{C}^{1}$. The proof of $\hat{\iota} \cdot \psi=\mathrm{q}^{k} \phi \cdot \iota$ is essentially the same as that of Corollary 5.4 , hence is omitted.

Finally the last assertion follows immediately from Proposition 4.9. q.e.d.

Thus the (local) contact equivalence of involutive submanifolds of $J^{k}($ (E) satisfying (c.1) and (c.2) is reduced to the (local) equivalence of the contact systems of order $k$ associated with them.

\section{§. Geometrization of involutive systems}

5.1. Contact system of order $k$. In view of Proposition 4.4, we first give the following definition.

Definition 5.1. Let $R$ be a manifold. A contact system of order $k$ of degree $n$ on $R$ is a family $\left\{D^{1}, \cdots, D^{k}\right\}$ of differential systems on $R$ satisfying the following conditions:

(1) $D^{1}$ and $D^{2}$ are differential systems of codimension 1 and $n+1$ respectively.

(2) The derived sheaf $\partial \mathscr{D}^{r+1}$ of $D^{r+1}$ is a subsheaf of the sheaf $\mathscr{D}^{r}$ of $D^{r}$ for $r=1, \cdots, k-1$.

(3) The assignment $x \mapsto F^{r}(x)=C h\left(D^{1}\right)(x) \cap \cdots \cap C h\left(D^{r}\right)(x)$ defines a subbundle $F^{r}$ of $D^{r+1}$ of codimension $n$ for $r=1, \cdots, k-1$.

(4) $\quad F^{k}(x)=C h\left(D^{1}\right)(x) \cap \cdots C h\left(D^{k}\right)(x)=\{0\}$ at each $x \in R$.

(5) If $k \geqq 3, F^{k-1}=F^{1} \cap D^{k}$.

The manifold $R$ together with the contact system of order $k$ of degree $n$ is called a PD manifold of order $k$ of degree $n$.

First, for the contact system of order $k$ of degree $n$, we note

Lemma 5.2. (i ) $D^{1} \supset D^{2} \supset \cdots \supset D^{k}$.

(ii) $F^{r}=F^{r-1} \cap D^{r+1}$ for $r=2, \cdots, k-1$. 
Proof. (i) is clear by Definition 5.1 (2). In order to prove (ii), we first have

$$
F^{k-1}=F^{k-1} \cap D^{k} \subset F^{k-2} \cap D^{k} \subset \cdots \cap F^{1} \cap D^{k}=F^{k-1} .
$$

Hence we get $F^{r-1}=F^{r} \cap D^{k}$ for $r=1, \cdots, k-2$. Let $x$ be any point of $R$. Then, from $D^{r}(x) \supset D^{r+1}(x), F^{r}(x) \subset D^{r+1}(x)$ and $F^{r}(x) \cap D^{k}(x)=F^{k-1}(x)$, we have

$$
\begin{aligned}
\operatorname{dim} F^{r}(x) & =\sum_{s=r+1}^{k-1} \operatorname{dim} F^{r}(x) \cap D^{s}(x) / F^{r}(x) \cap D^{s+1}(x)+\operatorname{dim} F^{r}(x) \cap D^{k}(x) \\
& \leqq \sum_{s=r+1}^{k-1} \operatorname{dim} D^{s}(x) / D^{s+1}(x)+\operatorname{dim} F^{k-1}(x) .
\end{aligned}
$$

On the other hand, from Definition 5.1 (3), we see that the both sides of the above inequality equal to $\operatorname{dim} D^{r+1}(x)-n$. Hence we get

$$
\operatorname{dim} F^{r}(x) / F^{r}(x) \cap D^{r+2}(x)=\operatorname{dim} D^{r+1}(x) / D^{r+2}(x),
$$

which implies $\operatorname{dim} F^{r}(x) \cap D^{r+2}(x)=\operatorname{dim} D^{r+2}(x)-n$. Since $F^{r+1}$ is a subbundle of $D^{r+2}$ of codimension $n$, we obtain $F^{r+1}(x)=F^{r}(x) \cap D^{r+2}(x)$ for $r=1, \cdots$, $k-2$.

$$
\text { q.e.d. }
$$

Let $\left(R ; D^{1}, \cdots, D^{k}\right)$ be a PD manifold of order $k$ of degree $n$. By (1) and (3) of Definition 5.1, $F^{1}=C h\left(D^{1}\right)$ is a completely integrable differential system on $R$ of codimension $2 n+1$. Let $K=R / F^{1}$ be the set of all leaves of the foliation defined by $F^{1}$, and let $\bar{\rho}_{1}$ be the natural projection of $R$ onto $K$. We say that $R$ is regular with respect to $F^{1}$ if there exists a differentiable structure on $K$ such that $\bar{\rho}_{1}$ is a submersion. It is well known that if $R$ is regular with respect to $F^{1}$, such a differentiable structure on $K$ is unique and is called the quotient manifold structure (cf. Theorem XIV [17, p. 28]). Furthermore it is also well known that if $R$ is regular with respect to $F^{1}=C h\left(D^{1}\right)$, there exists a unique differential system $C$ on $K$ such that $D^{1}=\left(\bar{\rho}_{1}\right)_{*}^{-1}(C)$ (cf. Théorèm [7, p. 52]). It is obvious that $\widetilde{E}=(K, C)$ is a contact manifold of dimension $2 n+1$. $\quad$ ( 5 is called the associated contact manifold of $\left(R ; D^{1}, \cdots\right.$, $D^{k}$ ). One should note that a PD manifold $R$ is always locally regular with respect to $F^{1}$.

Now we will give the Realization Theorem for PD manifolds by using Lemma 1.5, which shows that a PD manifold $\left(R ; D^{1}, \cdots, D^{k}\right)$ can be realized locally as a submanifold of $J^{k}(\mathfrak{C})$ satisfying (c.1), (c.2) and (c.3) such that $D^{r}$ $=\iota_{*}^{-1}\left(\partial^{k-r} C^{k}\right)$. In other words a PD manifold of order $k$ of degree $n$ is realized locally as a $k$-th order partial differential equation of one unknown function of $n$ independent variables in a canonical manner.

Theorem 5.3 (Realization Theorem). Let $\left(R ; D^{1}, \cdots, D^{k}\right)$ be a PD manifold of order $k$ of degree $n$ such that $R$ is regular with respect to $F^{1}$. Let $\Subset=$ 
$(K, C)$ be the associated contact manifold of $\left(R ; D^{1}, \cdots, D^{k}\right)$ with the projection $\bar{\rho}_{1} ; R \rightarrow K$ and let $\left(J^{r}(\mathcal{C}), C^{r}\right)$ be the $r$-th prolongation of $C(r=2, \cdots, k)$.

Then there exists a unique map c of $R$ into $J^{k}($ (5) satisfying the following two conditions:

(1) $\rho_{1}^{k} \cdot \iota=\bar{\rho}_{1}$.

(2) $D^{r}=\iota_{*}^{-1}\left(\partial^{k-r} C^{k}\right)$ for $r=2, \cdots, k$.

Furthermore, let $\bar{\rho}_{r}=\rho_{r}^{k} \cdot \iota(r=2, \cdots, k)$. Then, for each $r=2, \cdots, k, \bar{\rho}_{r}$ : $R \rightarrow J^{r}(\mathfrak{S})$ satisfies the following;

(i ) $r \quad D^{r}=\left(\bar{\rho}_{r}\right)_{*}^{-1}\left(C^{r}\right)$ and $\rho_{r-1}^{r} \cdot \bar{\rho}_{r}=\bar{\rho}_{r-1}$,

(ii) $\operatorname{Ker}\left(\bar{\rho}_{r}\right)=F^{r}$,

(iii) ${ }_{r} \quad \bar{\rho}_{r}(x)=\left(\bar{\rho}_{r-1}\right)_{*}\left(D^{r}(x)\right)$ as a point of $J\left(J^{r-1}(\mathfrak{S}), n\right)$ for $x \in R$.

In particular $\mathrm{is}$ an immersion and $F^{r}$ is completely integrable for $r=1$, $\cdots, k$.

We will call $\iota$ the canonical immersion of $\left(R ; D^{1}, \cdots, D^{k}\right)$ into $J^{k}(\Im)$.

Proof. First, starting from $\bar{\rho}_{1}$, we will construct a unique map $\bar{\rho}_{r}: R \rightarrow$ $J^{r}(\mathfrak{C})$ satisfying the condition (i) $r$ for $r=2, \cdots, k$ by induction on $r$. For $r=1$, we have $D^{1}=\left(\bar{\rho}_{1}\right)_{*}^{-1}(C)$ and $\operatorname{Ker}\left(\bar{\rho}_{1}\right)_{*}=F^{1}$. Then $\left(R, D^{2}, \bar{\rho}_{1}, K\right)$ satisfies the assumptions of Lemma 1.5. Hence, by Lemma 1.5, there exists a unique map $\tilde{\rho}_{2}: R \rightarrow J(K, n)$ satisfying $D^{2}=\left(\tilde{\rho}_{2}\right)_{*}^{-1}\left(C_{2}^{*}\right)$ and $\Pi^{2} \cdot \tilde{\rho}_{2}=\bar{\rho}_{1}$, where $\Pi^{2}$ is the projection of $J(K, n)$ onto $K$ and $C_{2}^{*}$ is the canonical system on $J(K, n)$. In fact, for each $x \in R, \tilde{\rho}_{2}$ is defined by $\tilde{\rho}_{2}(x)=\left(\bar{\rho}_{1}\right)_{*}\left(D^{2}(x)\right)$ as a point of $J(K, n)$ and satisfies $\operatorname{Ker}\left(\left(\tilde{\rho}_{2}\right)_{*}\right)_{x}=F^{1}(x) \cap C h\left(D^{2}\right)(x)=F^{2}(x)$. On the other hand, from $\partial \mathscr{D}^{2} \subset \mathscr{D}^{1}$ and $D^{1}=\left(\bar{\rho}_{1}\right)_{*}^{-1}(C)$, we see that $\tilde{\rho}_{2}(x)$ is an integral element of $(K, C)$, i.e., $\tilde{\rho}_{2}(R) \in J^{2}(\mathfrak{S})$. Therefore we get a unique map $\bar{\rho}_{2}: R \rightarrow J^{2}(\mathfrak{S})$ satisfying (i) $)_{2}$. Furthermore $\bar{\rho}_{2}$ satisfies (ii) $)_{2}$ and (iii) $)_{2}$.

Now suppose that we have constructed a unique man $\bar{\rho}_{r}: R \rightarrow J^{r}($ (5) satisfying (i) $)_{r}$ such that (ii) $)_{r}$ and (iii) $)_{r}$ hold. Since $\operatorname{Ker}\left(\bar{\rho}_{r}\right)_{*}=F^{r}$ is a subbundle of $D^{r+1}$ of codimension $n,\left(R, D^{r+1}, \bar{\rho}_{r}, J^{r}(\mathfrak{S})\right)$ satisfies the assumptions of Lemma 1.5. Hence, by Lemma 1.5, there exists a unique map $\tilde{\rho}_{r+1}: R \rightarrow$ $J\left(J^{r}(\mathfrak{S}), n\right)$ satisfying $D^{r+1}=\left(\tilde{\rho}_{r+1}\right)_{*}^{-1}\left(C_{r+1}^{*}\right)$ and $\Pi^{r+1} \cdot \tilde{\rho}_{r+1}=\bar{\rho}_{r}$, where $\Pi^{r+1}$ is the projection of $J\left(J^{r}(\mathbb{E}), n\right)$ onto $J^{r}(\mathfrak{S})$ and $C_{r+1}^{*}$ is the canonical system on $J\left(J^{r}(\subseteq), n\right)$. In fact, for each $x \in R, \tilde{\rho}_{r+1}$ is defined by $\tilde{\rho}_{r+1}(x)=\left(\bar{\rho}_{r}\right)_{*}\left(D^{r+1}(x)\right)$ as a point of $J\left(J^{r}(\widetilde{C}), n\right)$ and satisfies $\operatorname{Ker}\left(\left(\tilde{\rho}_{r+1}\right)_{*}\right)_{x}=F^{r}(x) \cap C h\left(D^{r+1}(x)\right)=F^{r+1}(x)$. Hence it suffices to show $\tilde{\rho}_{r+1}(R) \subset J^{r+1}(\mathfrak{S})$. First, from $\partial \mathscr{D}^{r+1} \subset \mathscr{D}^{r}$ and $D^{r}=$ $\left(\bar{\rho}_{r}\right)_{*}^{-1}\left(C^{r}\right)$, we see that $\tilde{\rho}_{r+1}(x)$ is an integral element of $\left(J^{r}(\mathfrak{S}), C^{r}\right)$. On the other hand, by Lemma 5.2 (ii), we have $F^{r}=F^{r-1} \cap D^{r+1}$, i.e., $\operatorname{Ker}\left(\bar{\rho}_{r}\right)_{*}=F^{r-1}$ $\cap D^{r+1}$. Furthermore, from $\rho_{r-1}^{r} \cdot \bar{\rho}_{r}=\bar{\rho}_{r-1}$ and $Q^{r}=\operatorname{Ker}\left(\rho_{r-1}^{r}\right)_{*}$, we get $F^{r-1}=$ $\left(\bar{\rho}_{r}\right)_{*}^{-1}\left(Q^{r}\right)$. Hence we get $\left(\bar{\rho}_{r}\right)_{*}\left(D^{r+1}(x)\right) \cap Q^{r}\left(\bar{\rho}_{r}(x)\right)=\{0\}$. Therefore $\tilde{\rho}_{r+1}(x) \in$ $J^{r+1}(\mathfrak{S})$. Thus we obtain a unique map $\bar{\rho}_{r+1}: R \rightarrow J^{r+1}(\mathfrak{S})$ satisfying $(\mathrm{i})_{r+1}$. Furthermore $\bar{\rho}_{r+1}$ satisfies (ii) $)_{r+1}$ and (iii) $)_{r+1}$. 
Accordingly, for $r=k$, we get a unique map $\bar{\rho}_{k}: R \rightarrow J^{k}($ (5) satisfying (i) such that (ii) $)_{k}$ and (iii) $)_{k}$ hold. Since $\operatorname{Ker}\left(\bar{\rho}_{k}\right)_{*}=F^{k}=\{0\}, \bar{\rho}_{k}$ is an immersion of $R$ into $J^{k}(\S)$. From $\rho_{r-1}^{r} \cdot \bar{\rho}_{r}=\bar{\rho}_{r-1}$ for $r=2, \cdots, k$, we get $\rho_{r}^{k} \cdot \bar{\rho}_{k}=\bar{\rho}_{r}$ for $r=1$, $\cdots, k$. Furthermore, by Proposition 2.5, we have $\partial^{k-r} C^{k}=\left(\rho_{r}^{k}\right)_{*}^{-1}\left(C^{r}\right)$. Hence we obtain $D^{r}=\left(\bar{\rho}_{r}\right)_{*}^{-1}\left(C^{r}\right)=\left(\bar{\rho}_{k}\right)_{*}^{-1}\left(\partial^{k-r} C^{k}\right)$ for $r=1, \cdots, k$. Thus we have shown the existence of an immersion $\bar{\rho}_{k}: R \rightarrow J^{k}(\mathfrak{C})$ satisfying (1) and (2) such that $\bar{\rho}_{r}=\rho_{r}^{k} \cdot \bar{\rho}_{k}$ satisfies (i) $)_{r}$ (ii) $)_{r}$ and (iii) $)_{r}$ for $r=2, \cdots, k$.

Finally let us prove the uniqueness of $\iota$. Let $\iota$ be a map of $R$ into $J^{k}$ (ङ) satisfying (1) and (2). Set $\iota_{r}=\rho_{r}^{k} \cdot \iota$ for $r=1, \cdots, k$. First, from $\partial^{k-\tau} C^{k}=$ $\left(\rho_{r}^{k}\right)_{*}^{-1}\left(C^{r}\right)$, we get $D^{r}=\left(\iota_{r}\right)_{*}^{-1}\left(C^{r}\right)$ for $r=2, \cdots, k$. Furthermore we have $\rho_{r-1}^{r} \cdot \iota_{r}$ $=\rho_{r-1}^{k} \bullet \iota=\iota_{r-1}$ for $r=2, \cdots, k$. Hence $\iota_{r}$ is a map of $R$ into $J^{r}(\widetilde{S})$ satisfying $D^{r}=\left(\iota_{r}\right)_{*}^{-1}\left(C^{r}\right)$ and $\rho_{r-1}^{r} \cdot \iota_{r} \cdot=\iota_{r-1}$ for $r=2, \cdots, k$. Then, from $\iota_{1}=\bar{\rho}_{1}$ and the uniqueness of a map $\bar{\rho}_{r}: R \rightarrow J^{r}$ (ङ) satisfying (i) , we get $\iota_{r}=\bar{\rho}_{r}$ for $r=2, \cdots, k$ by induction on $r$. Thus we obtain $\iota=\bar{\rho}_{k}$, which shows the uniqueness of $\iota$.

q.e.d.

By Proposition 4.4 and Theorem 5.3, we see that a submanifold $R$ of $J^{k}(\sqrt{5})$ has the induced structure of a PD manifold if and only if $R$ satisfies the conditions (c.1), (c.2) and (c.3).

Corollary 5.4. Let $\left(R, D^{1}, \cdots, D^{k}\right)$ and $\left(\hat{R} ; \hat{D}, \cdots, \hat{D}^{k}\right)$ be PD manifolds of order $k$ of degree $n$ such that $R$ (resp. $\hat{R}$ ) is regular with respect to $F^{1}$ (resp. $\left.\hat{F}^{1}\right)$. Let $\mathfrak{C}$ and $\hat{\mathbb{C}}$ be contact manifolds associated with $R$ and $\hat{R}$ respectively. Let $\left(J^{k}(\mathfrak{S}), C^{k}\right)$ and $\left(J^{k}(\hat{\mathbb{C}}), \hat{C}^{k}\right)$ be the $k$-th order prolongations of $\mathfrak{S}$ and $\hat{\mathbb{E}}$ respectively.

Then an isomorphism $\psi$ of $\left(R ; D^{1}, \cdots, D^{k}\right)$ onto $\left(\hat{R} ; \hat{D}^{1}, \cdots, \hat{D}^{k}\right)$ induces a unique contact transformation $\phi$ of $\mathbb{E}$ onto $\hat{\mathbb{C}}$ such that $\hat{\iota} \cdot \psi=\mathrm{q}^{k} \dot{\phi} \cdot \iota$, where $\iota($ resp. $\hat{\imath})$ is the canonical immersion of $R($ resp. $\hat{R})$ into $J^{k}(\mathfrak{S})\left(\right.$ resp. $\left.J^{k}(\hat{\mathfrak{S}})\right)$ and $\mathrm{q}^{k} \phi$ is the $k$-th order prolongation of $\phi$.

Proof. Since $\psi$ is an isomorphism of $\left(R ; D^{1}, \cdots, D^{k}\right)$ onto $\left(\hat{R} ; \hat{D}^{1}, \cdots\right.$, $\left.\hat{D}^{k}\right)$, we have $\psi_{*}\left(D^{1}\right)=\hat{D}^{1}$. Hence, from $F^{1}=C h\left(D^{1}\right)$ and $\hat{F}^{1}=C h\left(\hat{D}^{1}\right)$, we get $\psi_{*}\left(F^{1}\right)=\hat{F}^{1}$. Therefore $\psi$ induces a unique diffeomorphism $\phi$ of $J^{1}(\mathfrak{S})$ onto $J^{1}(\hat{\mathfrak{C}})$ such that $\hat{\bar{\rho}}_{1} \cdot \psi=\phi \cdot \bar{\rho}_{1}$. By $D^{1}=\left(\bar{\rho}_{1}\right)_{*}^{-1}\left(C^{1}\right)$ and $\hat{D}^{1}=\left(\hat{\bar{\rho}}_{1}\right)_{*}^{-1}\left(\hat{C}^{1}\right), \phi$ is a contact transformation of $\left(\mathfrak{S}\right.$ onto $\hat{\mathfrak{C}}$. Set $\bar{\imath}=\left(\mathrm{q}^{k} \phi\right)^{-1} \cdot \hat{\imath} \cdot \psi$. Then it is easy to see that $\tilde{\imath}$ is a map of $R$ into $J^{k}(\mathfrak{C})$ satisfying $\rho_{1}^{k} \cdot \bar{\imath}=\bar{\rho}_{1}$ and $D^{r}=\bar{\iota}_{*}^{-1}\left(\partial^{k-r} C^{k}\right)$ for $r=$ $1, \cdots, k$. Therefore, by the uniqueness of the canonical immersion $\iota$ of $R$ into $J^{k}(\Im)$, we obtain $\iota=\bar{\iota}$, i.e., $\hat{\iota} \cdot \psi=\mathrm{q}^{k} \phi \cdot \iota$.

q.e.d.

5.2. Symbol algebra of a PD manifold. Let $\left(R ; D^{1}, \cdots, D^{k}\right)$ be a PD manifold of order $k$ of degree $n$. By Definition 5.1 (2), we can consider the graded algebra of $\left(R ; D^{1}, \cdots, D^{k}\right)$ at $x \in R$ (cf. 1.3 of $\S 1$ ).

DeFinition 5.5. Let $\left(R ; D^{1}, \cdots, D^{k}\right)$ be a PD manifold of order $k$ of 
degree $n$. Let $x$ be any point of $R$. The graded algebra $\xi(x)=\sum_{p=-1}^{-(k+1)} \mathfrak{g}_{p}(x)$ of $\left(R ; D^{1}, \cdots, D^{k}\right)$ at $x$ is called the symbol algebra of $\left(R ; D^{1}, \cdots, D^{k}\right)$ at $x \in R$.

For the symbol algebra $\xi(x)$ of $\left(R ; D^{1}, \cdots, D^{k}\right)$ at $x$, we have $\xi_{-1}(x)=$ $D^{k}(x)$ by definition (cf. 1.3 of $\S 1$ ). Hence $f(x)=F^{k-1}(x)$ is a subspace of $\xi_{-1}(x)$.

LEMMA 5.6. ( i ) $\operatorname{dim} \mathfrak{g}_{-(k+1)}(x)=1$ and $\operatorname{dim} \mathfrak{S}_{-k}(x)=n$.

(ii) $\left[\mathfrak{s}_{p}(x), \mathfrak{s}_{q}(x)\right]=\{0\}$ if $p, q<-1$.

(iii) If $X \in \mathfrak{S}_{p}(x)$ and $\left[X, \mathfrak{S}_{-1}(x)\right]=0$, then $X=0(-k \leqq p \leqq-2)$.

(iv) $\partial \mathscr{D}_{x}^{r}=\mathscr{D}_{x}^{r}+\left[\mathscr{D}_{x}^{r}, \mathscr{D}_{x}^{k}\right]$ for $r=1, \cdots, k$. Hence $\left[\mathfrak{s}(x), \mathfrak{S}_{-1}(x)\right]=\mathfrak{Z}_{p-1}(x)$ if and only if $D^{p+k}(x)=\partial D^{p+k+1}(x)$ for $p=-1, \cdots,-k$.

(v) $f(x)$ is an abelian subalgebra of $\xi(x)$ and is a subspace of $\xi_{-1}(x)$ of codimension $n$.

(vi) $f(x)=\left\{X \in \mathfrak{g}_{-1}(x) \mid\left[X, \mathfrak{g}_{p}(X)\right]=0\right.$ for $\left.p<-r\right\}$ for $r=1, \cdots, k-1$. In particular $\left[\mathfrak{f}(x), \mathfrak{\Xi}_{p}(x)\right]=\{0\}$ if $p<-1$.

(vii) If $X \in \mathfrak{f}(x)$ and $\left[X, \mathfrak{B}_{-1}(x)\right]=0$, then $X=0$.

Proof. (i) The assertion is clear by Definition 5.1.

(ii) The aassertion follows immediately from the definition of $\mathfrak{s}(x)$ (cf. 1.3 of $\S 1$ ).

(iii) Since $F^{k-1}$ is a subbundle of $D^{k}$ of codimension $n$, we can choose $X_{1}, \cdots, X_{n} \in \mathscr{D}_{x}^{k}$ such that $\mathscr{D}_{x}^{k}=\left\langle X_{1}, \cdots, X_{n}\right\rangle \oplus \mathscr{F}_{x}^{k-1}$ (direct sum of $\mathscr{R}_{x}$-modules), where $\left\langle X_{1}, \cdots, X_{n}\right\rangle$ is the $\mathscr{R}_{x}$-submodule of $\mathscr{D}_{x}^{k}$ generated by $X_{1} \cdots, X_{n}$. Furthermore $F^{r-1}$ is a subbundle of $D^{r}$ of codimension $n$. Then, from $F^{r-1} \cap$ $D^{k}=F^{k-1}$ and $D^{k} \subset D^{r}$, it is easy to see that $\mathscr{D}_{x}^{r}=\left\langle X_{1}, \cdots, X_{n}\right\rangle \oplus \mathscr{F}_{x}^{r-1}$ (direct sum of $\mathscr{R}_{x}$-modules), where $\mathscr{F}^{r-1}$ is the sheaf of $F^{r-1}$. Let $Y$ be any element of $\mathscr{D}_{x}^{r}$ such that $\left[Y, \mathscr{D}_{x}^{k}\right] \subset \mathscr{D}_{x}^{r}(1 \leqq r \leqq k-1)$. Let $Y=Y_{1}+Y_{2}$ be the decomposition according to the direct sum decomposition $\mathscr{D}_{x}^{r}=\left\langle X_{1}, \cdots, X_{n}\right\rangle \oplus \mathscr{F}_{x}^{r-1}$. Then, from $\left[Y_{1}, X_{i}\right] \in \mathscr{D}_{x}^{k-1} \subset \mathscr{D}_{x}^{r}$, we have $\left[Y_{2}, X_{i}\right] \in \mathscr{D}_{x}^{r}$ for $i=1, \cdots, n$. On the other hand, since $F^{r-1}$ is completely integrable (Theorem 5.3), we have $\left[\mathscr{F}_{x}^{r-1}, \mathscr{F}_{x}^{r-1}\right] \subset \mathscr{F F}_{x}^{r-1}$. Hence we get $\left[Y_{2}, \mathscr{D}_{x}^{r}\right] \subset \mathscr{D}_{x}^{r}$, i.e., $Y_{2} \in C h\left(\mathscr{D}^{r}\right)_{x}$. It follows that $Y_{2} \in \mathscr{F}_{x}^{r-1} \cap C h\left(\mathscr{D}^{r}\right)_{x} \subset \mathscr{F}_{x}^{r}$ (cf. 1.4 of $\S 1$ ). Therefore, we obtain $Y \in\left\langle X_{1}\right.$, $\cdots, X_{n}>\oplus \mathscr{F}_{x}^{r}=\mathscr{D}_{x}^{r+1}$. Thus (iii) is proved.

(iv) Let $X_{1}, \cdots, X_{n}$ be the same as in the proof of (iii). Then $\mathscr{D}_{x}^{r}=\left\langle X_{1}\right.$, $\left.\cdots, X_{n}\right\rangle \oplus \mathscr{F}_{x}^{r-1}$ for $r=1, \cdots, k$. Hence, from $\left[\mathscr{F}_{x}^{r-1}, \mathscr{F}_{x}^{r-1}\right] \subset \mathscr{F}_{x}^{r-1}, \partial \mathscr{D}_{x}^{r}=\mathscr{D}_{x}^{r}+$ $\left[\mathscr{D}_{x}^{r}, \mathscr{D}_{x}^{r}\right]$ is generated by $X_{i},\left[X_{i}, X_{j}\right],\left[X_{i}, \mathscr{F}_{x}^{r-1}\right](i=1, \cdots, n)$ and $\mathscr{F}_{x}^{r-1}$. Therefore, we get $\partial \mathscr{D}_{x}^{r} \subset \mathscr{D}_{x}^{r}+\left[\mathscr{D}_{x}^{r}, \mathscr{D}_{x}^{k}\right]$. Opposite inclusion is obvious.

(v) Since $F^{k-1}$ is a completely integrable subbundle of $D^{k}$ of codimension $n$, we get the assertion.

(vi) By Lemma 5.2 (ii), we have $F^{k-1}=F^{r} \cap D^{k}$ for $r=1, \cdots, k-2$. Hence in order to prove the assertion, we have only to show that, for $X \in$ 
$\mathfrak{g}_{-1}(x)=D^{k}(x),\left[X, \mathfrak{g}_{p}(x)\right]=0$ if and only if $X \in D^{k}(x) \cap C h\left(D^{p+k+1}\right)(x)$ for $p=-2$, $\cdots,-k$. Let $X$ be any vector of $D^{k}(x)$. Let $X_{1}, \cdots, X_{r}$ (resp. $\omega_{1}, \cdots, \omega_{s}$ ) be a free basis of $\mathscr{D}_{x}^{p+k+1}$ (resp. $\left.\left(\mathscr{D}^{p+k+1}\right)_{x}^{\perp}\right)$. Then, from $D^{k} \subset D^{p+k+1}$ and the proof of Lemma 1.2, we see that $X \in C h\left(D^{p+k+1}\right)(x)$ if and only if $\left.\left(X_{i}\right\lrcorner \mathrm{d} \omega_{j}\right)_{x}(X)=0$ for $i=1, \cdots, r$ and $j=1, \cdots, s$. By the definition of the bracket operation of $\mathcal{E}(x)$, this implies that $X \in C h\left(D^{p+k+1}\right)(x)$ if and only if $\left[X, \mathfrak{g}_{p}(x)\right]=0$. Thus we obtain (vi).

(vii) The assertion is equivalent to $F^{k}(x)=F^{k-1}(x) \cap C h\left(D^{k}\right)(x)=\{0\}$.

q.e.d.

Digressing from the symbol algebra $\mathfrak{s}(x)$, we now consider a graded Lie algebra $\mathfrak{S}=\sum_{p=-1}^{-(k+1)} \mathfrak{S}_{p}$ satisfying the following four conditions:

(a.1) $\left[\mathfrak{g}_{p}, \mathfrak{s}_{q}\right]=0$ if $p, q<-1$.

(a.2) If $X \in \mathfrak{g}_{p}$ and $\left[X, \mathfrak{g}_{-1}\right]=0$, then $X=0(-k \leqq p \leqq-2)$.

(a.3) $\mathfrak{f}=\left\{X \in \mathfrak{g}_{-1} \mid\left[X, \mathfrak{g}_{p}\right]=0\right.$ for every $\left.p<-1\right\}$ is an abelian subalgebra of $s$.

(a.4) If $X \in \mathfrak{f}$ and $\left[X, \mathfrak{S}_{-1}\right]=0$, then $X=0$.

Set $\mathfrak{g}_{-(k+1)}=W_{s}$ and $\mathfrak{g}_{-1} / \mathfrak{f}=V_{s}$. Let $\kappa$ be the projection of $\mathfrak{g}_{-1}$ onto $V_{s}$. Let $\tilde{\alpha}_{p}(-k \leqq p \leqq-1)$ be the bilinear map of $\mathfrak{g}_{p} \times \otimes^{p+k+1} \mathfrak{g}_{-1}$ into $W_{s}=\mathfrak{g}_{-(k+1)}$ defined by

$$
\tilde{\alpha}_{p}\left(Y_{p}, X_{1}, \cdots, X_{p+k+1}\right)=\left[\left[\cdots\left[Y_{p}, X_{1}\right], \cdots\right], X_{p+k+1}\right] \in W_{s},
$$

for $Y_{p} \in \mathfrak{S}_{p}$ and $X_{i} \in \mathfrak{S}_{-1}(i=1, \cdots, p+k+1)$. Then, from (a.1), it follows that $\tilde{\alpha}_{p}(-k \leqq p \leqq-2)$ is symmetric with respect to $X_{1}, \cdots, X_{p+k+1}$, i.e., $\tilde{\alpha}_{p}$ defines the bilinear map $\alpha_{p}: \mathfrak{g}_{p} \times S^{p+k+1}\left(\mathfrak{g}_{-1}\right) \rightarrow W_{s}$. Furthermore, from (a.3), $\alpha_{p}$ induces a unique bilinear map $\beta_{p}: \mathfrak{s}_{p} \times S^{p+k+1}\left(V_{s}\right) \rightarrow W_{s}$ such that $\alpha_{p}=\beta_{p} \cdot \mu_{p}$, where $\mu_{p}$ $=i d_{\bar{x}_{p}} \times \otimes^{p+k+1} \kappa$ (cf. The proof of Lemma $3.7(\mathrm{v})$ ). Similarly, from (a.1) and (a.3), $\tilde{\alpha}_{-1}$ defines the bilinear map $\alpha_{-1}: \mathfrak{\uparrow} \times S^{k}\left(\mathfrak{g}_{-1}\right) \rightarrow W_{s}$ and $\alpha_{-1}$ induces a unique bilinear map $\beta_{-1}: \mathfrak{f} \times S^{k}\left(V_{s}\right) \rightarrow W_{s}$ such that $\alpha_{-1}=\beta_{-1} \cdot \mu_{-1}$, where $\mu_{-1}=$ $i d_{\mathrm{f}} \times \otimes{ }^{k} \kappa$.

Let $\beta_{p}^{*}(-k \leqq p \leqq-2)$ (resp. $\left.\beta_{-1}^{*}\right)$ be the linear map of $g_{p}$ (resp. f) into $W_{s} \otimes S^{p+k+1}\left(V_{s}^{*}\right)\left(\right.$ resp. $\left.W_{s} \otimes S^{k}\left(V_{s}^{*}\right)\right)$ defined by

$$
\beta_{p}^{*}\left(Y_{p}\right)\left(\tilde{X}_{1} \bigcirc \cdots @ \tilde{X}_{p+k+1}\right)=\beta_{p}\left(Y_{p}, \tilde{X}_{1} \bigcirc \cdots\left(\tilde{X}_{p+k+1}\right)(-k \leqq p \leqq-1),\right.
$$

for $Y_{-1} \in \mathfrak{f}, Y_{p} \in \mathfrak{P}_{p}(p \leqq-2)$ and $\tilde{X}_{i} \in V_{s}(i=1, \cdots, p+k+1)$. Then we have

Proposition 5.7. Let $\mathfrak{g}=\sum_{p=-1}^{-(k+1)} \mathfrak{g}_{p}$ be a graded Lie algebra satisfying (a.1), (a.2), (a.3) and (a.4). Let $W_{s}=\mathfrak{g}_{-(k+1)}, V_{s}=\mathfrak{g}_{-1} / \mathfrak{f}$ and $\kappa, \beta_{p}^{*}$ be as above. Then

(1) $\beta_{-1}^{*}$ is a linear isomorphism of $\mathfrak{f}$ into $W_{s} \otimes S^{k}\left(V_{s}^{*}\right)$. And $\beta_{p}^{*}$ is a linear isomorphism of $\mathfrak{s}_{p}$ into $W_{s} \otimes S^{p+k+1}\left(V_{s}^{*}\right)$ for $p=-2, \cdots,-k$.

(2) There exists a graded Lie algebra isomorphism $\phi$ of $s$ into $\mathfrak{C}^{k}\left(V_{s}, W_{s}\right)$ 
satisfying the following two conditions;

(i ) $\phi_{-(k+1)}=i d_{W s}$,

(ii) $q \cdot \phi_{-1}=\kappa$,

where $\phi_{p}=\left.\phi\right|_{s_{p}}$ and $q$ is the projection of $\mathfrak{\Im}_{-1}^{k}\left(V_{s}, W_{s}\right)$ onto $V_{s}$ corresponding to $\mathfrak{S}_{-1}^{k}\left(V_{s}, W_{s}\right)=V_{s} \oplus \mathfrak{\Im}\left(V_{s}, W_{s}\right)$.

Let $\phi$ be a graded Lie algebra isomorphism of $g$ into $\mathfrak{C}^{k}\left(V_{s}, W_{s}\right)$ satisfying (i) and (ii). Then $\phi_{p}=\beta_{p}^{*}$ for $p=-2, \cdots,-k$ and $\left.\phi\right|_{\mathrm{f}}=\beta_{-1}^{*}$. Furthermore, let $\hat{\phi}$ be another graded Lie algebra isomorphism of $\xi$ into $\widetilde{C}^{k}\left(V_{s}, W_{s}\right)$ satisfying (i) and (ii). Then there exists a graded Lie algebra automorphism $A$ of ${ }^{(5}\left(V_{s}, W_{s}\right)$ such that $A \in N^{k}\left(V_{s}, W_{s}\right)$ and $\hat{\phi}=A \cdot \phi$.

(3) Let $V$ and $W$ be vector spaces such that $\operatorname{dim} V=\operatorname{dim} V_{s}$ and $\operatorname{dim} W=$ $\operatorname{dim} W_{s}$. Let $\phi$ and $\hat{\phi}$ be graded Lie algebra isomorphism of $\mathfrak{g}$ into ${ }^{k}{ }^{k}(V, W)$ such that $\mathfrak{f}=\phi^{-1}(\widetilde{\mho}(V, W))=\hat{\phi}^{-1}(\mathfrak{\mho}(V, W))$. Then there exists a graded Lie algebra automorphism $A$ of $\mathbb{S}^{k}(V, W)$ such that $\hat{\phi}=A \cdot \phi$.

Proof. (1) (a.2) and (a.4) imply that $\beta_{p}^{*}$ is injective for $p=-1, \cdots$, $-k$.

(2) First we will construct a graded Lie algebra isomorphism $\phi^{0}$ of $g$ into ${ }^{k}{ }^{k}\left(V_{s}, W_{s}\right)$ satisfying (i) and (ii). Let us fix, once for all, a linear subspace $V_{0}$ of $\xi_{-1}$ such that $\mathfrak{E}_{-1}=V_{0} \oplus \tilde{f}$ (direct sum). Then $\kappa_{0}=\left.\kappa\right|_{V_{0}}$ is a linear isomorphism of $V_{0}$ onto $V_{s}$. We define a linear map $\rho_{0}$ of $V_{s}$ into $W_{s} \otimes S^{k}\left(V_{s}^{*}\right)$ by setting

$$
\rho_{0}(X)\left(X_{1}, \cdots, X_{k}\right)=\frac{k}{(k+1) !} \sum_{\sigma \in \mathbb{E}_{k}}\left[\left[\cdots\left[\tilde{X}, \tilde{X}_{\sigma(1)}\right], \cdots\right], \tilde{X}_{\sigma(k)}\right] \in W_{s}
$$

where $\widetilde{\Im}_{k}$ is the permutation group of $k$ elements and $\tilde{X}=\kappa_{0}^{-1}(X), \tilde{X}_{i}=\kappa_{0}^{-1}\left(X_{i}\right)$ $\in V_{0}(i=1, \cdots, k)$. Then, from $\left[\left[Y_{p}, \tilde{X}_{1}\right], \tilde{X}_{2}\right]=\left[\left[Y_{p}, \tilde{X}_{2}\right], \tilde{X}_{1}\right]$ for $Y_{p} \in \mathcal{B}_{p}(p \leqq-2)$ and $\tilde{X}_{i} \in \mathfrak{P}_{-1}(i=1,2)$, we have

$$
\begin{aligned}
\rho_{0}(X)\left(X_{1}, \cdots, X_{k}\right)= & \frac{1}{k+1}\left\{\left[\left[\cdots\left[\tilde{X}, \tilde{X}_{1}\right], \cdots\right], \tilde{X}_{k}\right]\right. \\
& \left.+\frac{1}{(k-2) !} \sum_{\tau \in \mathfrak{E}_{k-1}}\left[\left[\cdots\left[\left[\left[\tilde{X}, \tilde{X}_{\tau(2)}\right], \tilde{X}_{1}\right], \tilde{X}_{\tau(3)}\right], \cdots\right], \tilde{X}_{\tau(k)}\right]\right\},
\end{aligned}
$$

where $\widetilde{S}_{k-1}$ is the permutation group of $2, \cdots, k$. By using the Jacobi identity, we easily get

$$
\left.\left.\left(X^{\prime}\right\lrcorner \rho_{0}(X)-X\right\lrcorner \rho_{0}\left(X^{\prime}\right)\right)\left(X_{1}, \cdots, X_{k-1}\right)=\left[\left[\cdots\left[\left[\tilde{X}, \tilde{X}^{\prime}\right], \tilde{X}_{1}\right], \cdots\right], \tilde{X}_{k-1}\right] .
$$

Hence we obtain

$$
\left.\left.\beta_{-2}^{*}\left(\left[\tilde{X}_{1}, \tilde{X}_{2}\right]\right)=X_{2}\right\lrcorner \rho_{0}\left(X_{1}\right)-X_{1}\right\lrcorner \rho_{0}\left(X_{2}\right)
$$


for $X_{1}, X_{2} \in V_{s}, \tilde{X}_{1}=\kappa_{0}^{-1}\left(X_{1}\right)$ and $\tilde{X}_{2}=\kappa_{0}^{-1}\left(X_{2}\right)$. Now we define a linear isomorphism $\phi^{0}$ of $g$ into $\mathfrak{C}^{k}\left(V_{s}, W_{s}\right)$ by setting

$$
\begin{array}{ll}
\phi_{-(k+1)}^{0}=i d_{W_{s}}, & \phi_{p}^{0}=\beta_{p}^{*}(-k \leqq p \leqq-2), \\
\left.\phi^{0}\right|_{\mathfrak{f}}=\beta_{-1}^{*}, & \left.\phi^{0}\right|_{V_{0}}=\kappa+\rho_{0} \cdot \kappa .
\end{array}
$$

Then, from (5.2), it is easy to see that $\phi^{0}$ is a graded Lie algebra homomorphism and satisfies (i) and (ii).

Let $\phi$ be a graded Lie algebra isomorphism of $\xi$ into $\mathcal{S}^{k}\left(V_{s}, W_{s}\right)$ satisfying (i) and (ii). From (ii), we see that $\phi_{-1}(\tilde{X})-\kappa(\tilde{X}) \in W_{s} \otimes S^{k}\left(V_{s}^{*}\right)$ for any $\tilde{X} \in \mathfrak{g}_{-1}$. Hence we get

$$
\begin{aligned}
\phi_{p}\left(Y_{p}\right)\left(X_{1}, \cdots, X_{p+k+1}\right) & =\left[\left[\cdots\left[\phi_{p}\left(Y_{p}\right), X_{1}\right], \cdots\right], X_{p+k+1}\right] \\
& =\left[\left[\cdots\left[\phi_{p}\left(Y_{p}\right), \phi_{-1}\left(\tilde{X}_{1}\right)\right], \cdots\right], \phi_{-1}\left(\tilde{X}_{p+k+1}\right)\right],
\end{aligned}
$$

for $Y_{p} \in \Xi_{p}(p \leqq-2), Y_{-1} \in \tilde{T}$ and $X_{i}=\kappa\left(\tilde{X}_{i}\right) \in V_{s}(i=1, \cdots, p+k+1)$. Furthermore, since $\phi$ is a homomorphism and $\phi_{-(k+1)}=i d_{W_{s}}$, we obtain

$$
\begin{aligned}
\phi_{p}\left(Y_{p}\right)\left(X_{1}, \cdots, X_{p+k+1}\right) & =\left[\left[\cdots\left[Y_{p}, \tilde{X}_{1}\right] \cdots\right], \tilde{X}_{p+k+1}\right] \\
& =\beta_{p}^{*}\left(Y_{p}\right)\left(X_{1}, \cdots, X_{p+k+1}\right),
\end{aligned}
$$

i.e., $\phi_{p}=\beta_{p}^{*}$ for $p=-2, \cdots,-k$ and $\left.\phi\right|_{f}=\beta_{-1}^{*}$.

In order to prove the last assertion, we have only to show that there exists a graded Lie algebra automorphism $A$ of $\mathcal{C}^{k}\left(V_{s}, W_{s}\right)$ such that $A \in N^{k}$ $\left(V_{s}, W_{s}\right)$ and $\phi^{0}=A \cdot \phi$. By (ii), we can define a linear map $\rho_{\phi}$ of $V_{s}$ into $W_{s} \otimes S^{k}\left(V_{s}^{*}\right)$ by setting

$$
\rho_{\phi}(X)=\phi_{-1}(\tilde{X})-X \quad \text { for } \quad X \in V_{s} \quad \text { and } \quad \tilde{X}=\kappa_{0}^{-1}(X) \in V_{0} .
$$

In particular $\rho_{0}=\rho_{\phi 0}$. Since $\phi$ is a homomorphism and $\phi_{-2}=\beta_{-2}^{*}$, we have

$$
\left.\beta_{-2}^{*}\left(\left[\tilde{X}_{1}, \tilde{X}_{2}\right]\right)=\left[\phi_{-1}\left(\tilde{X}_{1}\right), \phi_{-1}\left(\tilde{X}_{2}\right)\right]=X_{2}\right\lrcorner \rho_{\phi}\left(X_{1}\right)-X_{1}-\rho_{\phi}\left(X_{2}\right),
$$

for $\tilde{X}_{i} \in V_{0}$ and $X_{i}=\kappa\left(\tilde{X}_{i}\right)(i=1,2)$. Set $\rho=\rho_{0}-\rho_{\phi}$. Then $\rho: V_{s} \rightarrow W_{s} \otimes S^{k}\left(V_{s}^{*}\right)$ satisfies

$$
\left.X_{1}-\rho\left(X_{2}\right)=X_{2}\right\lrcorner \rho\left(X_{1}\right) \quad \text { for } X_{1}, X_{2} \in V_{s} \text {, }
$$

i.e., $\rho$ can be considered as an element of $W_{s} \otimes S^{k+1}\left(V_{s}^{*}\right)$. Now we define a linear isomorphism $A_{\rho}$ of $\mathcal{\complement}^{k}\left(V_{s}, W_{s}\right)$ onto $\mathcal{\complement}^{k}\left(V_{s}, W_{s}\right)$ by setting

$$
\left.A_{\rho}\right|_{W_{s}}=i d_{W_{s}},\left.\quad A_{\rho}\right|_{W_{s} \otimes S^{r}\left(V_{s}^{*}\right)}=i d_{W_{s} \otimes S^{r}\left(V_{s}^{*}\right)}(r=1, \cdots, k),\left.\quad A_{\rho}\right|_{V s}=i d_{V_{s}}+\rho .
$$

Then $A_{\rho}$ is a graded Lie algebra automorphism of $\widetilde{C}^{k}\left(V_{s}, W_{s}\right)$ such that $A_{\rho} \in$ $N^{k}\left(V_{s}, W_{s}\right)$ (cf. the proof of Proposition $3.7(1)$ ). Obviously we have 


$$
\begin{array}{ll}
A_{\rho} \cdot \phi(Y)=Y=\phi^{0}(Y) & \text { for } Y \in W_{s}=\mathfrak{I}_{-(k+1)}, \\
A_{\rho} \cdot \phi\left(Y_{p}\right)=\beta_{p}^{*}\left(Y_{p}\right)=\phi^{0}\left(Y_{p}\right) & \text { for } Y_{-1} \in \mathfrak{f} \text { and } Y_{p} \in \mathfrak{g}_{p}(-2 \leqq p \leqq-k) .
\end{array}
$$

Furthermore we have

$$
A_{\rho} \cdot \phi(X)=\kappa(X)+\rho(\kappa(X))+\rho_{\dot{\phi}}(\kappa(X))=\phi^{0}(X) \quad \text { for } X \in V_{0} .
$$

Therefore we obtain $A_{\rho} \cdot \phi=\phi^{0}$.

(3) Let $\phi$ be a graded Lie algebra isomorphism of $\xi$ into ${ }^{k}(V, W)$ such that $\mathfrak{f}=\phi^{-1}(\widetilde{J}(V, W))$. In view of (2), we have only to show that there exists a graded Lie algebra isomorphism $\hat{A}$ of $\complement^{k}(V, W)$ onto ${ }^{k}\left(V_{s}, W_{s}\right)$ such that $\hat{\phi}=\hat{A} \cdot \phi: \mathfrak{Z} \rightarrow \mathfrak{S}^{k}\left(V_{s}, W_{s}\right)$ satisfies (i) and (ii). From $\tilde{\uparrow}=\phi^{-1}(\widetilde{\mho}(V, W))$, it follows that there exists a unique linear isomorphism $a$ of $V_{s}$ onto $V$ such that $a \cdot \kappa$ $=\hat{q} \cdot \phi_{-1}$, where $\hat{q}$ is the projection of $\mathbb{C}_{-1}^{k}(V, W)$ onto $V$ corresponding to $\widetilde{c}_{-1}^{k}(V, W)=V \oplus \mathfrak{\mho}(V, W)$. On the other hand $b=\phi_{-(k+1)}$ is a linear isomorphism of $W_{s}$ onto $W$. Let $\chi^{r}(a, b)(r=1, \cdots, k)$ be the linear isomorphism of $W_{s} \otimes S^{r}\left(V_{s}^{*}\right)$ onto $W \otimes S^{r}\left(V^{*}\right)$ defined by $\chi^{r}(a, b)=b \times \otimes \otimes^{r}\left(a^{*}\right)^{-1}$, where $a^{*}$ is the adjoint linear map of $a$. For $a$ and $b$, we construct a linear isomorphism $A=A(a, b)$ of $\mathbb{C}^{k}\left(V_{s}, W_{s}\right)$ onto $\mathbb{C}^{k}(V, W)$ by setting

$$
\left.A\right|_{V_{s}}=a,\left.A\right|_{W_{s}}=b \quad \text { and }\left.\quad A\right|_{W_{s} \otimes S^{r}\left(V_{s}^{*}\right)}=\chi^{r}(a, b) \quad \text { for } \quad r=1, \cdots, k .
$$

It is easy to see that $A$ is a graded Lie algebra isomorphism of ${ }^{k}{ }^{k}\left(V_{s}, W_{s}\right)$ onto $\mathcal{C}^{k}(V, W)$. We set $\hat{\phi}=A^{-1} \cdot \phi$. Then $\hat{\phi}$ is a graded lie algebra isomorphism of $\mathfrak{s}$ into $\widetilde{C}^{k}\left(V_{s}, W_{s}\right)$ such that $\hat{\phi}_{-(k+1)}=i d_{W_{s}}$ and $q \cdot \hat{\phi}_{-1}=\kappa . \quad$ q.e.d.

Corollary 5.8. Let $\mathfrak{u}=\sum_{p=-1}^{-(k+1)} \mathfrak{u}_{p}$ be a graded subalgebra of $\mathfrak{S}^{k}(V, W)$ such that $\mathfrak{u}_{-(k+1)}=W, \mathfrak{u}_{-k}=W \otimes V^{*}$ and $\mathfrak{u}_{-1} \supset V$. Set $\mathfrak{u}_{*}=\mathfrak{u}_{-1} \cap \widetilde{\mho}(V, W)$ and let $S(\mathfrak{u})$ be the set of abelian subalgebras $\hat{V}$ such that $\mathfrak{u}_{-1}=\hat{V} \oplus \mathfrak{u}_{*}$ (direct sum). Let $A(\mathfrak{u})$ be the group of graded Lie algebra automorphisms of $\mathfrak{u}$. Then

(1) Each $\phi \in A(\mathfrak{u})$ has a unique extension $\hat{\phi} \in A^{k}(V, W)$, i.e., $\left.\hat{\phi}\right|_{\mathfrak{u}}=\phi$. Hence $A(\mathfrak{u})$ can be considered as a closed subgroup of $A^{k}(V, W)$ and is given by

$$
A(\mathfrak{u})=\left\{\phi \in A^{k}(V, W) \mid \phi(\mathfrak{u})=\mathfrak{u}\right\} .
$$

(2) $N(\mathfrak{u})=A(\mathfrak{u}) \cap N^{k}(V, W)$ is a closed normal subgroup of $A(\mathfrak{u})$ and is canonically isomorphic with the vector group $p u_{*}$. Furthermore $N(\mathfrak{i t})$ acts simply transitively on $S(\mathfrak{H})$.

(3) $A(\mathfrak{u})=G(\mathfrak{u}) \cdot N(\mathfrak{u})($ semi-direct product $)$, where $G(\mathfrak{u})=A(\mathfrak{u}) \cap G^{k}(V, W)$.

(4) $N(\mathfrak{u})=\left\{\phi \in A(\mathfrak{u})|\phi|_{\mathfrak{u}_{p}}=i d_{\mathfrak{u}_{p}}\right.$ for $\left.p<-1\right\}$.

Proof. (1) By $\mathfrak{u}_{-k}=W \otimes V^{*}$, we have $\mathfrak{u}_{*}=\left\{X \in \mathfrak{u}_{-1} \mid\left[X, \mathfrak{u}_{-k}\right]=0\right\}$.

Hence $\mathfrak{u}$ satisfies the conditions (a.1), (a.2), (a.3) and (a.4) with $\mathfrak{f}=\mathfrak{H}_{*}$. Let $\iota$ be the inclusion map of $\mathfrak{u}$ into $\mathfrak{S}^{k}(V, W)$. Let $\phi \in A(\mathfrak{u})$. Then $\iota$ and $\iota \cdot \phi$ are 
graded Lie algebra isomorphism of $\mathfrak{u}$ into $\mathfrak{C}^{k}(V, W)$ such that $\mathfrak{u}_{*}=\iota^{-1}(\mathfrak{\mho}(V, W))$ $=(\iota \cdot \phi)^{-1}(\mathfrak{\mho}(V, W))$. Hence, by Proposition $5.7(3)$, there exists $\hat{\phi} \in A^{k}(V, W)$ such that $\iota \cdot \phi=\hat{\phi} \cdot \iota$, i.e., $\left.\hat{\phi}\right|_{\mathfrak{u}}=\phi$.

(2) For $A_{\rho} \in N^{k}(V, W), A_{\rho}(\mathfrak{u})=\mathfrak{u}$ if and only if $\rho(V) \subset \mathfrak{H}_{*}$, i.e., if and only if $\rho \in p \mathfrak{u}_{*}$. Furthermore $S(\mathfrak{u})=\left\{\hat{V} \in S^{k+1}(V, W) \mid \hat{V} \subset \mathfrak{u}_{-1}\right\}$. Hence the assertion follows immediately from Proposition 3.7 (1).

(3) Let $\phi \in A(\mathfrak{u})$. By Proposition 3.7 (2), there exist $\phi^{\prime} \in G^{k}(V, W)$ and $A_{\rho_{\phi}} \in N^{k}(V, W)$ such that $\phi=\phi^{\prime} \cdot A_{\rho_{\phi}}$. From $\left.A_{\rho_{\phi}}\right|_{W \otimes S^{r}\left(V^{*}\right)}=i d_{W \otimes S^{r}\left(V^{*}\right)}(r=0, \cdots$, $k)$, it follows that $\phi^{\prime}\left(\mathfrak{u}_{p}\right)=\mathfrak{u}_{p}(-(k+1) \leqq p \leqq-2)$ and $\phi^{\prime}\left(\mathfrak{u}_{*}\right)=\mathfrak{u}_{*}$. Furthermore, by $\mathfrak{u}_{-1} \supset V$, we have $\phi^{\prime}(\mathfrak{u})=\mathfrak{u}$, i.e., $\phi^{\prime} \in A(\mathfrak{u})$. Hence we obtain $\phi^{\prime} \in G(\mathfrak{u})$ and $A_{\rho \phi} \in N(\mathfrak{u})$. Therefore, the assertion follows immediately from Proposition $3.7(2)$.

(4) Let $\phi \in A(\mathfrak{u})$ such that $\left.\phi\right|_{\mathfrak{u}_{p}}=i d_{\mathfrak{u}_{p}}$ for $p<-1$. By (3), there exist $\phi^{\prime} \in G(\mathfrak{u})$ and $A_{\rho} \in N(\mathfrak{u})$ such that $\phi=\phi^{\prime} \cdot A_{\rho}$. Then, since $\left.A_{\rho}\right|_{\mathfrak{u}_{p}}=i d_{\mathfrak{u}_{p}}$ for $p<$ -1 , we have $\left.\phi^{\prime}\right|_{\mathfrak{u}_{p}}=i d_{\mathfrak{u}_{p}}$ for $p<-1$. From $\mathfrak{u}_{-k}=W \otimes V^{*}$ and $\mathfrak{u}_{*}=\left\{X \in \mathfrak{u}_{-1} \mid\right.$ $\left.\left[X, \mathfrak{u}_{-k}\right]=0\right\}$, it follows that $\phi^{\prime}(X)-X \in \mathfrak{H}_{*}$ for any $X \in V$, i.e., $\left.\phi^{\prime}\right|_{V}=i d_{V}$. Hence we get $\phi^{\prime}=i d_{A(\mathfrak{u})}$.

q.e.d.

REMARK 5.9. Notations being the same as in Corollary 5.8 , let $\mathfrak{u}_{0}$ be the Lie algebra of $A(\mathfrak{u})$. Let $\mathfrak{g}(\mathfrak{u})$ and $\mathfrak{n}(\mathfrak{u})$ be the subalgebras of $\mathfrak{u}_{0}$ corresponding to $G(\mathfrak{u})$ and $N(\mathfrak{l})$ respectively. Then, by (3) and (4) of Corollary 5.8, we have

$$
\begin{aligned}
& \mathfrak{H}_{0}=\mathfrak{g}(\mathfrak{H}) \oplus \mathfrak{H}(\mathfrak{H}) \text { (direct sum of vector spaces), } \\
& \mathfrak{H}(\mathfrak{H})=\left\{X \in \mathfrak{H}_{0} \mid\left[X, \mathfrak{H}_{p}\right]=0 \text { for } p<-1\right\} .
\end{aligned}
$$

Obviously $\mathfrak{n}(\mathfrak{u})$ can be considered as a subalgebra of $\mathfrak{g l}\left(\mathfrak{u}_{-1}\right)$ by $X \in \mathfrak{n}(\mathfrak{u}) \mapsto$ $\left.X\right|_{\mathfrak{u}_{-1}} \in \mathfrak{g} \mathfrak{l}\left(\mathfrak{u}_{-1}\right)$. Furthermore, by $(2), \mathfrak{n}(\mathfrak{u})$ is isomorphic with $p \mathfrak{u}_{*}$. This isomorphism $a: p \mathfrak{H}_{*} \rightarrow \mathfrak{n}(\mathfrak{u})$ is given as follows: Let $\rho \in p \mathfrak{u}_{*}$, i.e., $\rho$ is a linear map of $V$ into $\mathfrak{u}_{*} \subset W \otimes S^{k}\left(V^{*}\right)$ such that $\left.\left.X\right\lrcorner \rho(Y)=Y\right\lrcorner \rho(X)$ for $X, Y \in V$. Then $a(\rho)$ is defined by $a(\rho)(X)=\rho \cdot q(X) \in \mathfrak{H}_{*} \subset \mathfrak{H}_{-1}$ for $X \in \mathfrak{u}_{-1}$, where $q$ is the projection of $\mathfrak{u}_{-1}$ onto $V$ corresponding to $\mathfrak{u}_{-1}=V \oplus \mathfrak{u}_{*}$.

Let $p^{(r+1)} \mathfrak{H}_{*}$ be the $(r+1)$-th prolongation of $\mathfrak{u}_{*}$, i.e., $p^{(r+1)} \mathfrak{u}_{*}=\mathfrak{u}_{*} \otimes$ $S^{r+1}\left(V^{*}\right) \cap W \otimes S^{r+2}\left(V^{*}\right)$, and let $\mathfrak{n}^{(r)}(\mathfrak{u})$ be the $r$-th prolongation of $\mathfrak{n}(\mathfrak{u}) \subset$ $\mathfrak{g} \mathfrak{(}\left(\mathfrak{u}_{-1}\right)$, i.e., $\mathfrak{n}^{(r)}(\mathfrak{u})=\mathfrak{n}(\mathfrak{u}) \otimes S^{r}\left(\mathfrak{u}_{-1}^{*}\right) \cap \mathfrak{u}_{-1} \otimes S^{r+1}\left(\mathfrak{u}_{-1}^{*}\right)$. We consider a linear map $a^{(r)}$ of $p^{(r+1)} \mathfrak{u}_{*}$ into $\mathfrak{n}^{(r)}(\mathfrak{u})$ defined by

$$
a^{(r)}(\rho)\left(X_{1}, \cdots, X_{r}\right)=a\left(\rho\left(q\left(X_{1}\right), \cdots, q\left(X_{r}\right)\right)\right) \in \mathfrak{n}(\mathfrak{H})
$$

for $\rho \in p^{(r+1)} \mathfrak{H}_{*}$ and $X_{1}, \cdots, X_{r} \in \mathfrak{H}_{-1}$. Then it is easy to see that $a^{(r)}$ is a linear isomorphism of $p^{(r+1)} \mathfrak{H}_{*}$ onto $\mathfrak{n}^{(r)}(\mathfrak{H})$.

Assume that $\mathfrak{u}$ is generated, as a Lie algebra, by $\mathfrak{u}_{-1}$. Then $\mathfrak{u}$ is a fundamental graded algebra. One should note that, by Corollary $2[19 ;$ p. 76], 1 is of infinite type if and only if $\mathfrak{u}_{*}$ is of infinite type as a subspace of $W \otimes S^{k}\left(V^{*}\right)$. 
Now we study the structure of the symbol algebra $\mathfrak{s}(x)$ of $\left(R ; D^{1}, \cdots, D^{k}\right)$ at $x \in R$. By Lemma 5.6, $\Sigma(x)$ satisfies the conditions (a.1), (a.2), (a.3) and (a.4). Let $V(x)$ denote the factor space $\mathfrak{I}_{-1}(x) / \mathfrak{f}(x)=D^{k}(x) / F^{k-1}(x)$ and let $\kappa$ be the projection of $\mathfrak{g}_{-1}(x)$ onto $V(x)$. Then we can define the linear map $\tau_{p}(x)$ (resp. $\tau_{-1}(x)$ ) of $\mathfrak{g}_{p}(x)$ (resp. $\left.f(x)\right)$ into $\mathfrak{g}_{-(k+1)}(x) \otimes S^{p+k+1}\left(V(x)^{*}\right)\left(\right.$ resp. $\mathfrak{S}_{-(k+1)}(x)$ $\left.\otimes S^{k}\left(V(x)^{*}\right)\right)$ by setting

$$
\tau_{p}(x)\left(Y_{p}\right)\left(\tilde{X}_{1} \bigcirc \cdots\left(\tilde{X}_{p+k+1}\right)=\left[\left[\cdots\left[Y_{p}, X_{1}\right], \cdots\right], X_{p+k+1}\right](-k \leqq p \leqq-1),\right.
$$

for $Y_{-1} \in \mathfrak{f}(x), Y_{p} \in \mathfrak{g}_{p}(x)(p \leqq-2), X_{i} \in \mathfrak{Z}_{-1}(x)$ and $\tilde{X}_{i}=\kappa\left(X_{i}\right) \in V(x)(i=1, \cdots$, $p+k+1)$. Furthermore, we define the first prolongation $p \mathfrak{f}(x)$ of $f(x)$ by

$$
p \tilde{f}(x)=\tau_{-1}(x)(\mathfrak{f}(x)) \otimes V(x)^{*} \cap \mathfrak{z}_{-(k+1)}(x) \otimes S^{k+1}\left(V(x)^{*}\right) .
$$

Note that $p \digamma(x)$ is, by definition, a subspace of $\xi_{-(k+1)}(x) \otimes S^{k+1}\left(V(x)^{*}\right)$. Let $R^{(1)}(x)$ be the set of integral elements $w$ of $\left(R, D^{k}\right)$ at $x$ such that $\mathfrak{g}_{-1}(x)=w \oplus$ $f(x)$ (direct sum). Then we have

Proposition 5.10. Let $\mathfrak{g}(x)$ be the symbol algebra of $\left(R ; D^{1}, \cdots, D^{k}\right)$ at $x \in R$.

(1) $\tau_{-1}(x)$ is a linear isomorphism of $\mathrm{f}(x)$ into $\mathfrak{S}_{-(k+1)}(x) \otimes S^{k}\left(V(x)^{*}\right)$. $\tau_{p}(x)$ is a linear isomorphism of $\mathfrak{B}_{p}(x)$ into $\mathfrak{B}_{-(k+1)}(x) \otimes S^{p+k+1}\left(V(x)^{*}\right)$ for $p=-2$, $\cdots,-k$.

(2) There exists a graded Lie algebra isomorphism $\phi$ of $\mathfrak{\xi}(x)$ into $\mathfrak{\complement}^{k}(n)$. Furthermore, let $\hat{\phi}$ be another graded Lie algebra isomorphism of $\xi(x)$ into $\mathfrak{C}^{k}(n)$. Then there exists a graded Lie algebra automorphism $A$ of $\mathfrak{S}^{k}(n)$ such that $\hat{\phi}=A \cdot \phi$.

(3) If $R^{(1)}(x)$ is not empty, the vector group $p \tilde{f}(x)$ canonically acts simply transitively on $R^{(1)}(x)$.

Proof. (1) The assertion is clear by Proposition 5.7 (1).

(2) Let $V$ and $W$ be vector spaces of dimension $n$ and 1 respectively. Since $\operatorname{dim} \mathfrak{s}_{-(k+1)}(x)=1$ and $\operatorname{dim} V(x)=n$, the existence of an isomorphism $\phi$ of $\mathfrak{s}(x)$ into $\mathfrak{C}^{k}(n)=\mathfrak{C}^{k}(V, W)$ follows from Proposition 5.7 (2). Furthermore, from $\operatorname{dim} \mathfrak{I}_{-k}=\operatorname{dim} W \otimes V^{*}=n$ (Lemma $5.6(\mathrm{i})$ ), we have $\phi\left(\mathfrak{g}_{-k}(x)\right)=W \otimes V^{*}$. Hence, from $\mathrm{f}(x)=\left\{X \in \mathfrak{I}_{-1}(x) \mid\left[X, \mathfrak{I}_{-k}(x)\right]=0\right\}$. (Lemma 5.6 (vi)), easily get $\mathrm{f}(x)=$ $\phi^{-1}(\widetilde{\mho}(V, W))$. Therefore the assertion follows immediately from Proposition $5.7(3)$.

(3) The proof is quite similar to that of Proposition 3.9 (3). Let $w$ be any element of $R^{(1)}(x)$. Then, setting $V_{0}=w$ in the proof of Proposition 5.7 (2), we get an isomorphism $\phi_{w}$ of $\mathfrak{g}(x)$ into $\mathfrak{C}^{k}\left(V(x), \mathfrak{g}_{-(k+1)}(x)\right)$ such that $\phi_{w}(w)$ $=V(x)$. Let $\mathfrak{H}=\sum_{p=-1}^{-(k+1)} \mathfrak{H}_{p}$ be the graded subalgebra of $\mathfrak{C}^{k}\left(V(x), \mathfrak{g}_{-(k+1)}(x)\right)$ defined by $\mathfrak{u}_{-(k+1)}=\mathfrak{I}_{-(k+1)}(x), \mathfrak{u}_{p}=\tau_{p}(x)\left(\mathfrak{g}_{p}(x)\right)(p=-2, \cdots,-k)$ and $\mathfrak{u}_{-1}=V(x)$ 
$\oplus \tau_{-1}(x)(\mathfrak{f}(x))$. Then $\mathfrak{u}=\operatorname{Im} \phi_{w}$ for any $w \in R^{(1)}(x)$. Furthermore we see that, for $A_{\rho} \in N^{k}\left(V(x), \mathfrak{S}_{-(k+1)}(x)\right), A_{\rho}(\mathfrak{u})=\mathfrak{u}$ if and only if $\rho(V(x)) \subset \tau_{-1}(x)(\mathfrak{f}(x))$, i.e., if and only if $\rho \in p \nmid(x)$. Let $\rho$ be any element of $p f(x)$. Then we see that $w^{\prime}$ $=\left(A_{\rho} \cdot \phi_{w}\right)^{-1}(V(x)) \in R^{(1)}(x)$ and $A_{\rho} \cdot \phi_{w}=\phi_{w^{\prime}}$. Since $\phi_{w}=\phi_{w^{\prime}}$ if and only if $w=$ $w^{\prime}$, we have thus defined the action of $p f(x)$ on $R^{(1)}(x)$. Let $w_{1}$ and $w_{2}$ be any pair of elements of $R^{(1)}(x)$. Then, since $\phi_{w_{i}}$ satisfies the conditions (i) and (ii) of Proposition 5.7, there exists $A_{\rho} \in N^{k}\left(V(x), \mathfrak{Z}_{-(k+1)}(x)\right)$ such that $\phi_{w_{2}}=$ $A_{\rho} \cdot \phi_{w_{1}}$. Obviously $A_{\rho}(\mathfrak{u})=\mathfrak{u}$. Hence $p \mathfrak{f}(x)$ acts simply transitively on $R^{(1)}$.

q.e.d.

$\tau_{-1}(x)$ (resp. $\tau_{p}(x)$ ) is called the fundamental identification of $\mathrm{f}(x)$ (resp. $\left.\xi_{p}(x)\right)$ at $x \in R$.

Furthermore, by Lemma 5.6 (iv), we have

Proposition 5.11. Let $\left(R ; D^{1}, \cdots, D^{k}\right)$ be a PD manifold of order $k$ of degree $n$ and let $\Xi(x)$ be the symbol algebra at $x \in R$. Let $\tau_{p}=\tau_{p}(x)(-k \leqq p \leqq-1)$ and $R^{(1)}(x)$ be as above.

(1) $D^{r}(x)=\partial D^{r+1}(x)$ if and only if $V(x)-\tau_{r-k}\left(\mathfrak{g}_{r-k}(x)\right)=\tau_{r-k-1}\left(\mathfrak{g}_{r-k-1}(x)\right)$ for $r=1, \cdots, k-2$. Furthermore, if $R^{(1)}(x)$ is not empty, $D^{k-1}(x)=\partial D^{k}(x)$ if and only if $V(x)-\tau_{-1}(\mathfrak{f}(x))=\tau_{-2}\left(\mathfrak{B}_{-2}(x)\right)$.

(2) If $k \geqq 3$, then $D^{1}(x)=\partial D^{2}(x)$ if and only if $C h\left(D^{2}\right)(x)=F^{2}(x)$. Furthermore, if $k=2$ and $R^{(1)}(x)$ is not empty, then $D^{1}(x)=\partial D^{2}(x)$ if and only if $C h\left(D^{2}\right)$ $(x)=\{0\}$.

Proof. (1) Let us fix a linear subspace $V_{0}$ of $\mathfrak{g}_{-1}(x)$ such that $\mathfrak{g}_{-1}(x)=$ $V_{0} \oplus f(x)$ (direct sum). Then, as in the proof of Proposition 5.7, we get a graded Lie algebra isomorphism $\phi^{0}$ of $\mathfrak{g}(x)$ into $\mathfrak{C}^{k}\left(V(x), \mathfrak{Z}_{-(k+1)}(x)\right)$ such that $\left.\phi^{0}\right|_{\xi_{-(k+1)}(x)}=i d_{\xi_{-(k+1)}(x)},\left.\phi^{0}\right|_{\hat{p}_{p}(x)}=\tau_{p}$ for $p=-2, \cdots,-k$ and $\left.\phi^{0}\right|_{\tilde{f}(x)}=\tau_{-1}$. Hence we have

$$
\phi^{0}\left(\left[\mathfrak{g}_{p}(x), \mathfrak{Z}_{-1}(x)\right]\right)=\left[\tau_{p}\left(\mathfrak{g}_{p}(x)\right), \phi^{0}\left(\mathfrak{g}_{-1}(x)\right)\right]=\left[\tau_{p}\left(\mathfrak{g}_{p}(x)\right), V(x)\right]
$$

for $p<-1$. Furthermore, if $R^{(1)}(x)$ is not empty, we can take $V_{0} \in R^{(1)}(x)$. Then $\phi^{0}\left(V_{0}\right)=V(x)$. Hence we have

$$
\phi^{0}\left(\left[\mathfrak{F}_{-1}(x), \mathfrak{I}_{-1}(x)\right]\right)=\left[V(x), \tau_{-1}(\mathfrak{f}(x))\right] .
$$

Therefore, the assertions follow immediately from Lemma 5.6 (iv).

(2) $C h\left(D^{2}\right)(x)=F^{2}(x)$ if and only if $C h\left(D^{2}\right)(x) \subset C h\left(D^{1}\right)(x)$. Hence, the only if part follows from Lemma 1.3. In order to prove the if part, we will show that, if $D^{1}(x) \supsetneq \partial D^{2}(x)$, then $C h\left(D^{2}\right)(x) \supsetneq F^{2}(x)$. Assume that $D^{1}(x) \supsetneq$ $\partial D^{2}(x)$. Then, by (1) and Lemma 5.6 (i), we have

$$
H=V(x)-\tau_{-k+1}\left(\mathfrak{I}_{-k+1}(x)\right) \subsetneq \tau_{-k}\left(\mathfrak{I}_{-k}(x)\right)=\mathfrak{I}_{-(k+1)}(x) \otimes V(x)^{*}
$$


provided that $k \geqq 3$. And if $k=2$ and $R^{(1)}(x)$ is not empty, we have

$$
H=V(x) \downarrow \tau_{-1}(\mathfrak{f}(x)) \subseteq \mathfrak{g}_{-3}(x) \otimes V(x)^{*} .
$$

Since $\operatorname{dim} \mathfrak{s}_{-(x+1)}(x)=1$, we see that, in both cases, $H^{\perp}=\left\{X \in V(x) \mid X_{-\lrcorner} H=0\right\}$ is a non-trivial subspace of $V(x)$. Let $X$ be any non-zero vector of $H^{\perp}$. Now we proceed according as $k \geqq 3$ or $k=2$. First, in case $k \geqq 3$, let $\tilde{X}$ be any vector of $\mathfrak{\zeta}_{-1}(x)$ such that $k(\tilde{X})=X$. Then we easily get $\left[\tilde{X}, \mathfrak{\zeta}_{-k+1}(x)\right]=0$ and $X \in f(x)$. Therefore, by the proof of Lemma 5.6 (vi), we have $X \in C h\left(D^{2}\right)(x) \cap D^{k}(x)$ and $X \notin F^{2}(x) \cap D^{k}(x)$. In case $k=2$ and $R^{(1)}(x)$ is not empty, take $V_{0} \in R^{(1)}(x)$ and let $\tilde{X}$ be the unique vector of $V_{0}$ such that $\kappa(\tilde{X})=X$. Then we get $[\tilde{X}, f(x)]=$ 0 and $\tilde{X} \notin f(x)$. Since $V_{0}$ is an abelian subalgebra such that $\mathfrak{g}_{-1}(x)=V_{0} \oplus \tilde{f}(x)$, we obtain $\left[\tilde{X}, \mathfrak{g}_{-1}(x)\right]=0$, i.e., $\tilde{X} \in C h\left(D^{2}\right)(x)$. Thus, in both cases, we have $C h\left(D^{2}\right)(x) \supsetneq F^{2}(x)$.

q.e.d.

5.3. Involutive PD manifolds. First we give the following definition.

Definition 5.12. Let $\left(R ; D^{1}, \cdots, D^{k}\right)$ be a PD manifold of order $k$ of degree $n$ and let $\zeta(x)$ be the symbol algebra at $x \in R$. Then $\left(R ; D^{1}, \cdots, D^{k}\right)$ is called involutive if and only if the following two conditions are satisfied:

(1) $\mathfrak{S}(x)$ is isomorphic, as a graded Lie algebra, with an involutive subalgebra of $\int^{k}(n)$ at each $x \in R$.

(2) $\operatorname{dim} p f(x)$ is constant on $R$.

Furthermore, if $\mathfrak{z}(x)(x \in R)$ are mutually isomorphic, $\left(R^{1} ; D, \cdots, D^{k}\right)$ is called regularly involutive.

One should note that a PD manifold $\left(R ; D^{1}, \cdots, D^{k}\right)$ is regularly involutive if and only if there exists an involutive subalgebra $\xi$ of $\mathcal{C}^{k}(n)$ such that $\mathfrak{g}(x)$ is isomorphic with $\mathfrak{g}$ at each $x \in R$. In this case $\left(R ; D^{1}, \cdots, D^{k}\right)$ is called a regularly involutive $\mathrm{PD}$ manifold of type $?$.

Let $\left(R ; D^{1}, \cdots, D^{k}\right)$ be a PD manifold of order $k$ of degree $n$. The first prolongation $R^{(1)}$ of $\left(R ; D^{1}, \cdots, D^{k}\right)$ is defined by

$$
R^{(1)}=\bigcup_{x \in R} R^{(1)}(x) \subset J(R, n),
$$

where $R^{(1)}(x)$ is the set of integral elements $w$ of $\left(R, D^{k}\right)$ at $x$ such that $D^{k}(x)$ $=w \oplus F^{k-1}(x)$ (direct sum). Let $\pi$ be the projection of $J(R, n)$ onto $R$ and let $\rho^{(1)}$ be the restriction of $\pi$ to $R^{(1)}$. Let $x$ be any point of $R$. Take an open neighborhood $U$ of $x$ such that $U$ is regular with respect to $F^{1}$. Now consider a PD manifold $\left(U ;\left.D^{1}\right|_{U}, \cdots,\left.D^{k}\right|_{U}\right)$ and let $\subseteq$ be its associated contact manifold. Taking $U$ sufficiently small, we may assume that the canonical immersion $\iota: U \rightarrow J^{k}(\mathfrak{S})$ is an imbedding. Then, by Proposition 4.9 and Proposition $5.10(2), \iota(U)$ is an involutive submanifold if and only if $\left(U ;\left.D^{1}\right|_{U}, \cdots\right.$, $\left.\left.D^{k}\right|_{U}\right)$ is involutive. Hence we have 
Proposition 5.13 (cf. Appendix [15]). Let $\left(R ; D^{1}, \cdots, D^{k}\right)$ be a PD manifold of order $k$ of degree $n$. Let $R^{(1)}$ be the first prolongation of $R$. Then $\left(R ; D^{1}, \cdots, D^{k}\right)$ is involutive if and only if $\rho^{(1)}: R^{(1)} \rightarrow R$ is onto and each $w \in R^{(1)}$ is an ordinary integral element of $\left(R, D^{k}\right)$.

Therefore, in the real analytic category, the involutiveness of $\left(R ; D^{1}, \cdots\right.$, $\left.D^{k}\right)$ guarantees, for each $w \in R^{(1)}$, the existence of an $n$-dimensional integral manifold $N$ of $\left(R, D^{k}\right)$ passing through $x=\rho^{(1)}(w)$ such that $T_{x}(N)=w$ and $T_{y}(N) \cap F^{k-1}(y)=\{0\}$ for $y \in N$.

Let $\left(R ; D^{1}, \cdots, D^{k}\right)$ be an involutive $P D$ manifold of order $k$ of degree $n$. Then, by Definition $5.12(2), S(R)=\bigcup_{x \in R} p f(x)$ is a vector bundle over $R$. Hence, from Proposition 5.10 (3), it follows that $R^{(1)}$ is an affine bundle over $R$ modeled on $S(R)$ (cf. Proposition 7.1 [9]). Therefore $R^{(1)}$ is a submanifold of $J(R, n)$ and $\rho^{(1)}$ is a submersion of $R^{(1)}$ onto $R$. Now we define differential systems $D^{(r)}(r=1, \cdots, k)$ on $R^{(1)}$ by setting

$$
D^{(r)}=\left(\rho^{(1)}\right)_{*}^{-1}\left(D^{r}\right)
$$

Furthermore, let $C^{*}$ be the canonical system on $J(R, n)$ and set

$$
D^{(k+1)}(w)=T_{w}(R) \cap C^{*}(w) \quad \text { for } w \in R^{(1)} .
$$

Then, since $\rho^{(1)}$ is a submersion, $F^{(k)}=\operatorname{Ker} \rho_{*}^{(1)}$ is a differential system on $R^{(1)}$ and $F^{(k)}(w)$ is a subspace of $D^{(k+1)}(w)$ of codimension $n$ at each $w \in R^{(1)}$. Hence $D^{(k+1)}$ is a differential system on $R^{(1)}$. Then we have

Lemma 5.14. $\left(D^{(1)}, \cdots, D^{(k+1)}\right)$ is a contact system of order $k+1$ of degree $n$ on $R^{(1)}$.

Proof. We must check the five conditions of Definition 5.1.

(1) From $D^{(1)}=\left(\rho^{(1)}\right)_{*}^{-1}\left(D^{1}\right)$ and $D^{(2)}=\left(\rho^{(1)}\right)_{*}^{-1}\left(D^{2}\right)$, it follows that $D^{(1)}$ and $D^{(2)}$ are differential systems of codimension 1 and $n+1$ respectively.

(2) Since each $w \in R^{(1)}$ is an integral element of $\left(R, D^{k}\right)$ and $D^{(k+1)}(w)$ $=\left(\rho^{(1)}\right)_{*}^{-1}(w)$, we see that $\partial \mathscr{D}^{(k+1)} \subset \mathscr{D}^{(k)}$. Furthermore, from $D^{(r)}=\left(\rho^{(1)}\right)_{*}^{-1}\left(D^{r}\right)$ $(1 \leqq r \leqq k)$ and $\partial \mathscr{D}^{r+1} \subset \mathscr{D}^{r}(1 \leqq r \leqq k-1)$, we get $\partial \mathscr{D}^{(r+1)} \subset \mathscr{D}^{(r)}$ for $r=1, \cdots, k-1$.

(3) From $D^{(r)}=\left(\rho^{(1)}\right)_{*}^{-1}\left(D^{r}\right)$, it is easy to see that

$$
C h\left(D^{(r)}\right)(w)=\left(\rho^{(1)}\right)_{*}^{-1}\left(C h\left(D^{r}\right)\left(\rho^{(1)}(w)\right)\right) \text { for } w \in R^{(1)} \text { and } r=1, \cdots, k .
$$

Hence we get $F^{(r)}=(\rho)_{*}^{-1}\left(F^{r}\right)$ for $r=1, \cdots, k-1$, where $F^{(r)}(w)=C h\left(D^{(1)}\right)(w) \cap$ $\cdots \cap C h\left(D^{(r)}\right)(w)$. Furthermore, from $C h\left(D^{1}\right)(x) \cap \cdots \cap C h\left(D^{k}\right)(x)=\{0\}$, we obtain $F^{(k)}(w)=C h\left(D^{(1)}\right)(w) \cap \cdots \cap C h\left(D^{(k)}\right)(w)$ for $w \in R^{(1)}$, where $F^{(k)}=$ $\operatorname{Ker} \rho_{*}^{(1)}$. Therefore $F^{(r)}$ is a subbundle of $D^{(r+1)}$ of codimension $n$ for $r=1$, $\cdots, k$. 
(4) $\left(R^{(1)}, D^{(k+1)}, \rho^{(1)}, R\right)$ satisfies the assumption of Lemma 1.5. On the other hand $R^{(1)}$ is a submanifold of $J(R, n)$. Hence, by Lemma $1.5, F^{(k)}(w) \cap$ $C h\left(D^{(k+1)}\right)(w)=\{0\}$ for $w \in R^{(1)}$.

(5) For each $w \in R^{(1)}$, we have $w \cap F^{k-1}\left(\rho^{(1)}(w)\right)=\{0\}$. Hence we get $D^{(k+1)} \cap F^{(k-1)}=F^{(k)}$. Furthermore, from $F^{k-1}=F^{1} \cap D^{k}$, we have $F^{(k-1)}=$ $F^{(1)} \cap D^{(k)}$. Therefore we obtain $F^{(k)}=F^{(1)} \cap D^{(k+1)}$.

q.e.d.

Assume further that $R$ is regular with respect to $F^{1}$ and let $\subseteq=(K, C)$ be its associated contact manifold. Let $\bar{\rho}_{1}$ be the projection of $R$ onto $J^{1}(\mathfrak{C})$. Then $\rho=\bar{\rho}_{1} \cdot \rho^{(1)}$ is a submersion of $R^{(1)}$ onto $J^{1}(\mathbb{E})$ and $F^{(1)}=\operatorname{Ker} \rho_{*}$. Since fibres of $\rho^{(1)} ; R^{(1)} \rightarrow R$ are connected, each fibre of $\rho ; R^{(1)} \rightarrow J^{1}(\mathfrak{S})$ is connected. Hence $R^{(1)}$ is regular with respect to $F^{(1)}$ and its associated contact manifold $\mathfrak{C}^{(1)}$ is naturally identified with $\mathfrak{S}$. Let $\iota$ be the canonical immersion of $R$ into $J^{k}(\mathbb{E})$. Then, as in the proof of Theorem 5.3, we see that there exists a unique immersion $\iota^{(1)}$ of $R^{(1)}$ into $J^{k+1}(\mathfrak{C})$ satisfying $D^{(k+1)}=\left(\iota^{(1)}\right)_{*}^{-1}\left(C^{k+1}\right)$ and $\iota \cdot \rho^{(1)}=\rho_{k}^{k+1} \cdot \iota^{(1)}$. Under the above identification of $\mathfrak{C}^{(1)}$ with $\mathfrak{C}, \iota^{(1)}$ is the canonical immersion of $R^{(1)}$ into $J^{k+1}$ (ङ).

Now we will consider the relations between symbol algebras of $R$ and $R^{(1)}$. Let $w$ be any point of $R^{(1)}$. Let $\mathfrak{g}^{(1)}(w)=\sum_{p=-1}^{-(k+2)} \mathfrak{g}_{p}^{(1)}(w)$ be the symbol algebra of $\left(R^{(1)} ; D^{(1)}, \cdots, D^{(k+1)}\right)$ at $w$ and let $\mathfrak{g}(x)=\sum_{p=-1}^{-(k+1)} \mathfrak{g}_{p}(x)$ be the symbol algebra of $\left(R ; D^{1}, \cdots, D^{k}\right)$ at $x=\rho^{(1)}(w)$. Recall that $\xi_{p}^{(1)}(w)=D^{(p+k+2)}(w) /$ $D^{(p+k+3)}(w)(-(k+2) \leqq p \leqq-2), \quad \mathfrak{I}_{-1}^{(1)}(w)=D^{(k+1)}(w), \quad \mathfrak{\xi}_{p}(x)=D^{p+k+1}(x) / D^{p+k+2}(x)$ $(-(k+1) \leqq p \leqq-2)$ and $\mathfrak{g}_{-1}(x)=D^{k}(x)$, where $D^{(0)}(w)=T_{w}\left(R^{(1)}\right)$ and $D^{0}(x)=$ $T_{x}(R)$. Hence $\rho_{*}^{(1)}$ induces the linear isomorphism $\sigma_{p}$ of $\mathfrak{g}_{p}^{(1)}(w)$ onto $\mathfrak{\xi}_{p+1}(x)$ satisfying the following commutative diagram for $p=-3, \cdots,-(k+2)$;

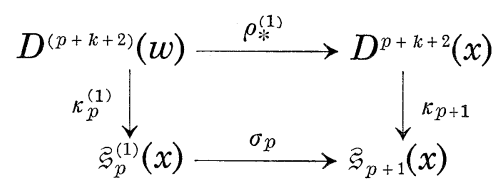

where vertical arrows are the natural projections. Furthermore we set $V(w)$ $=D^{(k+1)}(w) / F^{(k)}(w)$ and $V(x)=D^{k}(x) / F^{k-1}(x)$. Then $\rho_{*}^{(1)}$ induces the linear isomorphism $\sigma_{-2}$ of $\mathfrak{g}_{-2}^{(1)}(w)$ onto $f(x)=F^{k-1}(x)$ and the linear isomorphism $\sigma_{-1}$ of $V(w)$ onto $V(x)$ satisfying the following commutative diagrams;
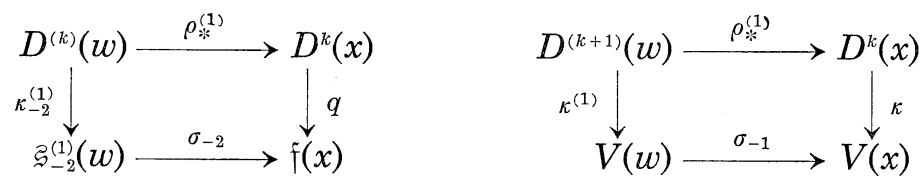

where $q$ is the projection corresponding to $D^{k}(x)=w \oplus f(x)$ and other vertical arrows are the natural projections. Hence $\hat{\sigma}_{r}=\sigma_{-(k+2)} \otimes \otimes^{r}\left(\sigma_{-1}^{*}\right)^{-1}$ is a linear isomorphism of $\mathfrak{g}_{-(k+2)}^{(1)}(w) \otimes S^{r}\left(V(w)^{*}\right)$ onto $\mathfrak{S}_{-(k+1)}(x) \otimes S^{r}\left(V(x)^{*}\right)$ for $r \geqq 1$, 
where $\sigma_{-1}^{*}$ is the adjoint linear map of $\sigma_{-1}$.

LEMMA 5.15. Notations being as above, let $\tau_{p}^{(1)}=\tau_{p}^{(1)}(w)\left(\right.$ resp. $\left.\tau_{p}=\tau_{p}(x)\right)$ be the fundamental identifications at $w \in R^{(1)}$ (resp. at $x \in R$ ).

(i ) $\sigma_{p-1}([Y, X])=\left[\sigma_{p}(Y), \rho_{*}^{(1)}(X)\right]$ for $Y \in \mathfrak{F}_{p}^{(1)}(w)$ and $X \in \mathfrak{S}_{-1}^{(1)}(w)(-(k+2)$ $\leqq p \leqq-2)$.

(ii) $\tau_{p+1} \cdot \sigma_{p}=\hat{\sigma}_{p+k+2} \cdot \tau_{p}^{(1)} \quad$ for $p=-2, \cdots,-(k+2)$.

(iii) $\hat{\sigma}_{k+1} \cdot \tau_{-1}^{(1)}\left(f^{(1)}(w)\right)=p f(x)$.

Proof. (i ) Take $Y^{\prime} \in \mathscr{D}_{w}^{(p+k+2)}$ and $X^{\prime} \in \mathscr{D}_{w}^{(k+1)}$ such that $Y=\kappa_{p}^{(1)}\left(Y_{w}^{\prime}\right)$ and $X=X_{w}^{\prime}$ Furthermore, take $Y^{\prime \prime} \in \mathscr{D}_{x}^{p+k+2}$ and $X^{\prime \prime} \in \mathscr{D}_{x}^{k}$ such that $Y_{x}^{\prime \prime}=\rho_{*}^{(1)}\left(Y_{w}^{\prime}\right)$ and $X_{x}^{\prime \prime}=\rho_{*}^{(1)}(X)$. Then we have $[Y, X]=\kappa_{p-1}^{(1)}\left(\left[Y^{\prime}, X^{\prime}\right]_{w}\right)$ and $\sigma_{p}(Y)=\kappa_{p+1}\left(Y_{x}^{\prime \prime}\right)$. Hence it suffices to show $\kappa_{p} \cdot \rho_{*}^{(1)}\left(\left[Y^{\prime}, X^{\prime}\right]_{w}\right)=\kappa_{p}\left(\left[Y^{\prime \prime}, X^{\prime \prime}\right]_{x}\right)$, i.e., $\rho_{*}^{(1)}\left(\left[Y^{\prime}, X^{\prime}\right]_{w}\right)-$ $\left[Y^{\prime \prime}, X^{\prime \prime}\right]_{x} \in D^{p+k+2}(x)$. Assume that $D^{p+k+2}$ is defined around $x$ by 1 -forms $\pi_{i}$ $(i=1, \cdots, \nu)$, where $\nu=\operatorname{codim} D^{(p+k+2)}$. Then $D^{(p+k+2)}$ is defined around $w$ by $\left(\rho^{(1)}\right)^{*} \pi_{i}(i=1, \cdots, \nu)$. Since $Y^{\prime}, X^{\prime} \in \mathscr{D}_{w}^{(p+k+2)}$ and $Y^{\prime \prime}, X^{\prime \prime} \in \mathscr{D}_{x}^{p+k+2}$, we get

$$
\pi_{i}\left(\rho_{*}^{(1)}\left(\left[Y^{\prime}, X^{\prime}\right]_{w}\right)-\left[Y^{\prime \prime}, X^{\prime \prime}\right]_{x}\right)=d \pi_{i}\left(Y_{x}^{\prime \prime}, X_{x}^{\prime \prime}\right)-d \pi_{i}\left(\rho_{*}^{(1)}\left(Y_{w}^{\prime}\right), \rho_{*}^{(1)}\left(X_{w}^{\prime}\right)\right)=0
$$

Thus we obtain $\sigma_{p-1}([Y, X])=\left[\sigma_{p}(Y), \rho_{*}^{(1)}(X)\right]$.

(ii) The assertion follows immediately from (i).

(iii) $\left[\mathfrak{g}_{-1}^{(1)}(w), \mathfrak{f}^{(1)}(w)\right] \subset \mathfrak{g}_{-2}^{(1)}(w)$ implies $\left.V(w)\right\lrcorner \tau_{-1}^{(1)}\left(f^{(1)}(w)\right) \subset \tau_{-2}^{(1)}\left(\mathfrak{g}_{-2}^{(1)}(w)\right)$. From (ii), we have $\hat{\sigma}_{k} \cdot \tau_{-2}^{(1)}\left(\mathcal{G}_{-2}^{(1)}(w)\right)=\tau_{-1} \cdot \sigma_{-2}\left(\mathcal{G}_{-2}^{(1)}(w)\right)=\tau_{-1}(\mathfrak{f}(x))$. Hence we get $\left.V(x)\right\lrcorner \hat{\sigma}_{k}$. $\tau_{-1}^{(1)}\left(f^{(1)}(w)\right) \subset \tau_{-1}(f(x))$, i.e., $\hat{\sigma}_{k} \cdot \tau_{-1}^{(1)}\left(\tilde{f}^{(1)}(w)\right) \subset p f(x)$. Since $R^{(1)}$ is an affine bundle modeled on $S(R)$, we have $\operatorname{dim} f^{(1)}(w)=\operatorname{dim} p f(x)$. Therefore we obtain $\hat{\sigma}_{k} \cdot \tau_{-1}^{(1)}\left(\tilde{f}^{(1)}(w)\right)=p \tilde{f}(x)$.

q.e.d.

Let $\xi=\sum_{p=-1}^{-(k+1)} \xi_{p}$ be an involutive subalgebra of $\Im^{k}(n)=\mathfrak{S}^{k}(V, W)$ such that $\mathfrak{g}_{-1} \supset V$. We define the first prolongation $\mathfrak{g}^{(1)}$ of $\mathfrak{g}_{\mathfrak{g}}$ as follows:

$$
\mathfrak{g}^{(1)}=\sum_{p=-1}^{-(k+2)} \mathfrak{g}_{p}^{(1)} \quad(\text { direct sum), }
$$

where

$$
\begin{aligned}
& \mathfrak{\xi}_{p}^{(1)}=\mathfrak{g}_{p+1} \subset W \otimes S^{p+k+2}\left(V^{*}\right)(-(k+2) \leqq p \leqq-3), \\
& \mathfrak{g}_{-2}^{(1)}=\mathfrak{g}_{*} \subset W \otimes S^{k}\left(V^{*}\right), \quad \mathfrak{g}_{-1}^{(1)}=V \oplus p \mathfrak{g}_{*} .
\end{aligned}
$$

It is easy to see that $\mathfrak{g}^{(1)}$ is an involutive subalgebra of $\int^{k+1}(n)$. Then, by Lemma 5.15 and Proposition 9.5 [15], we finally obtain

Proposition 5.16. Let $\left(R ; D^{1}, \cdots, D^{k}\right)$ be an involutive PD manifold of order $k$ of degree $n$. Then $\left(R^{(1)} ; D^{(1)}, \cdots, D^{(k+1}\right)$ is an involutive $P D$ manifold of order $k+1$ of degree $n$ and $\left(R^{(1)}, R, \rho^{(1)}\right)$ is an affine bundle modeled on $S(R)$. Furthemore, if $R$ is a regularly involutive PD manifold of type $\xi$, then $R^{(1)}$ is of type $\mathfrak{S}^{(1)}$. 
REMARK 5.17. In our terminology, the geometric contents of [5] and [6] may be formulated as the problem of (local) equivalence of regularly involutive PD manifolds $\left(R ; D^{1}, D^{2}\right)$ of order 2 of degree 2 or 3 . In fact E. Cartan first classified the structure formulae of such involutive systems (see [6, I]). This is essentially equivalent to the classification of types of $\left(R ; D^{1}, D^{2}\right)$, i.e., the classification of the symbol algebra $\xi(x)$ of $\left(R ; D^{1}, D^{2}\right)$ at $x \in R$ (cf. Remark 6.7 (2)). Hence, by Proposition 5.10 (2), this classification is equivalent to the classification of involutive subalgebras $\mathfrak{B}$ of $\mathfrak{C}^{2}(n)=\mathfrak{C}^{2}(V, W)$ satisfying $\mathfrak{g}_{-3}=W$ and $\mathfrak{g}_{-2}=W \otimes V^{*}$ by the action of $A^{2}(V, W)$. Furthermore, by Proposition 3.7, this amounts to the classification of involutive subspaces of $S^{2}\left(V^{*}\right)$ by the action of $G L(V)$. In general the classification of involutive subalgebras of $\mathfrak{\complement}^{2}(n)$ of codimension 1 is equivalent to the classification of quadratic forms on $V$. And involutive subalgebras of codimension 2 are classified into two types (over $\boldsymbol{R}$ or $\boldsymbol{C}$ ) $(n \geqq 3)$.

REMARK 5.18. Let $\left(R ; D^{1}, \cdots, D^{k}\right)$ be a regularly involutive PD manifold of order $k$ of degree $n$ of type $\zeta$. Assume that $\xi$ is generated, as a Lie algebra, by $g_{-1}$. Then, from Proposition 5.11, it follows that $D^{r}=\partial^{k-r} D^{k}$ for $r=1, \cdots$, $k-1$. Furthermore, by Lemma 5.6 (iv), we obtain $D^{r}=\partial^{(k-r)} D^{k}$ for $r=1, \cdots$, $k-1$. Therefore $D^{k}$ is a regular differential system on $R$ of type 3. One should note that, by Remark 5.9 and Lemma 7.3., $\xi$ is a fundamental graded algebra of infinite type in the sense of N. Tanaka [19].

\section{§6. Higher order contact manifolds} nition.

6.1. Higher order contact manifolds. First we give the following defi-

Definition 6.1. Let $C$ be a differential system on a manifold $K$. Then $(K, C)$ is called a contact manifold of order $k$ of degree $n$ if and only if the following conditions are satisfied:

(1) There exists a family $\left\{C^{1}, \cdots, C^{k}\right\}$ of differential systems on $K$ such that $C^{k}=C$ and $C^{r}=\partial C^{r+1}$ for $r=1, \cdots, k-1$, i.e., the $r$-th derived sheaf $\partial^{r} C$ of $C$ defines a differential system $\partial^{r} C=C^{k-r}$ for $r=1, \cdots, k-1$.

(2) $C^{1}$ is a differential system of codimension 1 .

(3) $C^{2}$ is a differential system of codimension $n+1$.

(4) The Cauchy-Cartan characteristic system $C h\left(C^{r}\right)$ of $C^{r}$ is a subbundle of $C^{r+1}$ of codimension $n$ for $r=1, \cdots, k-1$.

(5) $C h\left(C^{k}\right)(x)=\{0\}$ at each $x \in K$.

(6) $C h\left(C^{k-1}\right)=C h\left(C^{1}\right) \cap C^{k}(k \geqq 3)$.

( 7) $\operatorname{dim} K=\sum_{r=1}^{l_{n}}{ }_{n} H_{r}+n+1$, where ${ }_{n} H_{r}=\left(\begin{array}{c}n+r-1 \\ r\end{array}\right)$. 
One should note that, by Lemma $1.3,\left(C^{1}, \cdots, C^{k}\right)$ is a contact system of order $k$ of degree $n$ on $K$. Hence $\left(K ; C^{1}, \cdots, C^{k}\right)$ is a PD manifold of order $k$ of degree $n$. Therefore, by (7) and Theorem 5.3, it follows that $(K, C)$ is locally isomorphic with $\left(J^{k}(\mathfrak{C}), C^{k}\right)$, where $\sqrt{5}$ is a contact manifold of dimension $2 n+1$. Conversely Proposition 2.5 shows that $\left(J^{k}(\mathbb{C}), C^{k}\right)$ is a contact manifold of order $k$ of degree $n$. Thus we obtain

THEOREm 6.2. Let $C$ be a differential system on a manifold $K$. Then $(K, C)$ is a contact manifold of order $k$ of degree $n$ if and only if, at each $x \in K$, there exist functions $x_{1}, \cdots, x_{n}, z$ and $p_{I}\left(I \in \bigcup_{r=1}^{k} M_{r}\right)$ defined on a neighborhood $U$ of $x$ such that

(1) $p_{I}$ is symmetric with respect to $I$,

(2) the system of functions $x_{1}, \cdots, x_{n}, z$ and $p_{I}\left(I \in \Sigma_{k}\right)$ forms a coordinate system on $U$,

(3) $C$ is defined on $U$ by the following 1-forms;

$$
\begin{aligned}
& \hat{\pi}=\mathrm{d} z-\sum_{i=1}^{n} p_{i} \mathrm{~d} x_{i}, \\
& \hat{\pi}_{I}=\mathrm{d} p_{I}-\sum_{j=1}^{n} p_{I, j} \mathrm{~d} x_{j}\left(I \in \Sigma_{k-1}\right) .
\end{aligned}
$$

Theorem 6.2 is a generalization of the classical Darboux's theorem for contact manifolds. Furthermore, in case $n=1$, we note (cf. [8])

Proposition 6.3. Let $C$ be a differential system on a manifold $K$. Then $(K, C)$ is a contact manifold of order $k$ of degree 1 if and only if the following conditions are satisfied:

(1) $C$ is a differential system of rank 2.

(2) There exists a family $\left\{C^{1}, \cdots, C^{k}\right\}$ of differential systems on $K$ such that $C^{k}=C, C^{r}$ is a differential system of codimension $r$ and satisfies $\partial C^{r}=C^{r-1}$ for $r=1, \cdots, k$, where $C^{0}=T(K)$.

(3) If $k \geqq 3, \operatorname{dim} C h\left(C^{1}\right)(x) \cap C^{k}(x)$ is locally constant on $K$.

Proof. The only if part follows immediately from Definition 6.1. Assume that $(K, C)$ satisfies the above three conditions. We will check the conditions of Definition 6.1. Clearly we have only to check (4), (5) and (6) of Definition 6.1. Let $x$ be any point of $K$. By (2), there exist 1-forms $\pi_{1}, \cdots$, $\pi_{k}$ defined on a neighborhood $U$ of $x$ such that $C^{r}$ is defined on $U$ by $\pi_{1}, \cdots$, $\pi_{r}$ for $r=1, \cdots, k$. Since $C^{r}=\partial C^{r+1}$ for $r=0, \cdots, k-1$, we have

$$
\mathrm{d} \pi_{r} \equiv 0\left(\bmod \pi_{1}, \cdots, \pi_{r+1}\right) \quad \text { and } \quad \mathrm{d} \pi_{r} \neq 0\left(\bmod \pi_{1}, \cdots, \pi_{r}\right)
$$

for $r=1, \cdots, k-1$. Hence there exist 1-forms $\omega_{r}$ on $U$ such that

$$
\mathrm{d} \pi_{r} \equiv \omega_{r} \wedge \pi_{r+1}\left(\bmod \pi_{1}, \cdots, \pi_{r}\right) .
$$


Furthermore, from $C^{r}(y)=\partial C^{r+1}(y) \supsetneq C^{r+1}(y), y \in U$, and Lemma 1.1, we see that $\pi_{1}, \cdots, \pi_{r+1}$ and $\omega_{r}$ are linearly independent everywhere on $U$. Hence, by Lemma 1.2, it follows that $C h\left(C^{r}\right)$ is defined on $U$ by $\pi_{1}, \cdots, \pi_{r+1}$ and $\omega_{r}$. Therefore $C h\left(C^{r}\right)$ is a subbundle of $C^{r+1}$ of codimension 1 for $r=1, \cdots, k-1$. Similarly, by (1) and $\partial C^{k} \supsetneq C^{k}$, it is easy to see that $C h\left(C^{k}\right)=\{0\}$. Thus we get (4) and (5) of Definition 6.1.

By (2) and Lemma 1.3, we have $C h\left(C^{k-1}\right) \subset C h\left(C^{1}\right)$. Furthermore $C h\left(C^{k-1}\right)$ is a subbundle of $C^{k}$ of codimension 1 . Hence we get $C h\left(C^{k-1}\right) \subset C h\left(C^{1}\right) \cap C^{k}$. Therefore, by (1) and (3), taking $U$ sufficiently small, we have $C h\left(C^{1}\right) \cap C^{k}=$ $C h\left(C^{k-1}\right)$ or $C^{k}$ on $U$. Assume that $C h\left(C^{1}\right) \cap C^{k}=C^{k}$ on $U$, i.e., $C h\left(C^{1}\right) \supset C^{k}$ on $U$. Then, since $C h\left(C^{1}\right)$ is defined on $U$ by $\pi_{1}, \pi_{2}$ and $\omega_{1}, \omega_{1}$ belongs to $\left\langle\pi_{1}, \cdots\right.$, $\left.\pi_{k}\right\rangle$, where $\left\langle\pi_{1}, \cdots, \pi_{k}\right\rangle$ denotes the $\mathscr{R}(U)$-module generated by $\pi_{1}, \cdots, \pi_{k}$. On the other hand we have $d \pi_{1} \equiv \omega_{1} \wedge \pi_{2}\left(\bmod \pi_{1}\right)$. Hence we have $\omega_{1} \wedge d \pi_{2} \equiv 0$ $\left(\bmod \pi_{1}, \pi_{2}\right)$. Furthermore, from $\mathrm{d} \pi_{2} \equiv \omega_{2} \wedge \pi_{3}\left(\bmod \pi_{1}, \pi_{2}\right)$, we get $\omega_{1} \wedge \omega_{2} \wedge \pi_{3} \equiv 0$ $\left(\bmod \pi_{1}, \pi_{2}\right)$. Since $\pi_{1}, \pi_{2}, \pi_{3}$ and $\omega_{2}$ are linearly independent on $U$, $\omega_{1}$ belongs to $\left\langle\pi_{1}, \pi_{2}, \pi_{3}, \omega_{2}\right\rangle$. Therefore $\omega_{1}$ belongs to $\left\langle\pi_{1}, \pi_{2}, \pi_{3}\right\rangle$. Hence we have $C^{3} \subset$ $C h\left(C^{1}\right)$ on $U$. Since $C^{3}$ and $C h\left(C^{1}\right)$ are subbundles of $C^{2}$ of codimension 1 , we get $C^{3}=C h\left(C^{1}\right)$ on $U$, which implies that $C^{3}$ is completely integrable on $U$. This contradiction shows that $C h\left(C^{1}\right) \cap C^{k}=C h\left(C^{k-1}\right)$.

q.e.d.

Let $(K, C)$ be a contact manifold of order $k$ of degree $n$. Now we will mention facts about the prolongations of $(K, C)$. Let $x$ be any point of $K$ and let $f(x)=\sum_{p=-1}^{-(k+1)} \mathfrak{f}_{p}(x)$ be the symbol algebra of $\left(K ; C^{1}, \cdots, C^{k}\right)$ at $x$. Let $K^{(1)}$ be the first prolongation of $(K, C)$, i.e.,

$$
K^{(1)}=\bigcup_{x \in K} K^{(1)}(x)
$$

where $K^{(1)}(x)$ is the set of integral elements $w$ of $(K, C)$ at $x$ such that $C(x)=$ $w \oplus F(x)$ (direct sum), $F=C h(\partial C)$. We set $V(x)=C(x) / F(x)$. Then, by Definition $6.1(7)$ and Proposition $5.10(2), \mathfrak{f}(x)$ is isomorphic with $\mathbb{C}^{k}(n)$. Furthermore, from Lemma 5.6 (iv), it follows that $\partial^{r} C=\partial^{(r)} C$ for $r=1, \cdots, k$. Hence $f(x)$ coincides with the graded algebra of $(K, C)$ at $x$. Therefore $(K, C)$ is a regular differential system of type $\mathfrak{C}^{k}(n)$ (cf. Proposition 3.6). In particular $K^{(1)}(x)$ is not empty at each $x \in K$. Furthermore, by Proposition 5.10 (3), $K^{(1)}(x)$ is an affine space modeled on $\mathfrak{f}_{-(k+1)}(x) \otimes S^{k+1}\left(V(x)^{*}\right)$ (Proposition 3.9 (3)). Let $S(K)$ be the vector bundle over $K$ defined by

$$
S(K)=\bigcup_{x \in K} \mathfrak{f}_{-(k+1)}(x) \otimes S^{k+1}\left(V(x)^{*}\right) .
$$

Then $K^{(1)}$ is an affine bundle over $K$ modeled on $S(K)$. Let $\rho^{(1)}$ be the projection of $K^{(1)}$ onto $K$ and let $C^{(1)}$ be the restriction to $K$ of the canonical system on $J(K, n)$, i.e., $C^{(1)}(w)=\left(\rho^{(1)}\right)_{*}^{-1}(w)$ for $w \in K^{(1)}$. One should observe 
the following fact: Let $\left(R ; D^{1}, \cdots, D^{k}\right)$ be a PD manifold of order $k$ of degree $n$. Then $\operatorname{dim} R \leqq \sum_{r=1}^{k} H_{r}+n+1$ and the equality holds if and only if $\left(R, D^{k}\right)$ is a contact manifold of order $k$ of degree $n$. Then, by Lemma 5.14 or directly by Lemma 2.3 and Proposition 2.5, we obtain

Proposition 6.4. Let $(K, C)$ be a contact manifold of order $k$ of degree $n(k \geqq 2)$. Then the first prolongation $\left(K^{(1)}, C^{(1)}\right)$ of $(K, C)$ is a contact manifold of order $k+1$ of degree $n$ and $K^{(1)}$ is an affine bundle over $K$ modeled on $S(K)$.

One should note that, in this context, $\left(J^{k+1}(\mathbb{E}), C^{k+1}\right)$ is, by definition, the first prolongation of the contact manifold $\left(J^{k}(\Subset), C^{k}\right)$ of order $k$ of degree $n$ (cf. $\S 2)$.

6.2. Characterization by graded algebras. Let $C$ be a differential system on a manifold $K$. If $(K, C)$ is a contact manifold of order $k$ of degree $n$, then $(K, C)$ is a regular differential system of type $\widetilde{C}^{k}(n)$. Now we will study the converse of this fact and will characterize contact manifolds of higher order by their graded algebras. First we have

THEOREM 6.5. Let $K$ be a manifold of dimension $\sum_{r=1}^{k} H_{r}+n+1$. Let $C$ be a regular differential system on $K$ of type ${ }^{k}(n)$. Then $(K, C)$ is a contact manifold of order $k$ of degree $n$ if and only if $C^{1}=\partial C^{2}$, where $C^{1}=\partial^{(k-1)} C$ and $C^{2}=\partial^{(k-2)} C$.

Proof. The only if part follows from Proposition 5.10 (2) and Lemma 5.6 (iv), or directly from Theorem 6.2 and Proposition 2.5 (1).

Let $M\left(\varsigma^{k}(n)\right)$ be the simply connected Lie group with Lie algebra $\varsigma^{k}(n)=$ $\mathcal{C}^{k}(V, W)$. Let $\theta$ be the Maurer-Cartan form of $M\left(\complement^{k}(n)\right)$. As in $\S 3$, take a basis $w_{0}$ of $W$ and a basis $e_{1}, \cdots, e_{n}$ of $V$. Then we have a natural basis $\left\{w_{0}, e_{1}, \cdots, e_{n}, w_{0} \otimes e_{I}^{*}\left(I \in \Sigma_{k}\right)\right\}$ of $\mathcal{S}^{k}(V, W)$ (cf. $\left.\S 3\right)$. With respect to this basis we express $\theta$ as follows:

$$
\begin{gathered}
\theta_{-(k+1)}=\hat{\pi} \cdot w_{0}, \quad \theta_{p}=\sum_{I \in S p+k+1} \hat{\pi}_{I} \cdot w_{0} \otimes e_{I}^{*}(p=-2, \cdots,-k), \\
\theta_{V}=\sum_{i=1}^{k} \hat{\omega}_{i} \cdot e_{i}, \quad \theta_{\mathrm{f}}=\sum_{I \in S_{k}} \hat{\pi}_{I} \cdot w_{0} \otimes e_{I}^{*}, \quad \theta_{-1}=\theta_{V}+\theta_{\mathrm{f}},
\end{gathered}
$$

where $\theta_{p}$ denotes the $\widehat{C}_{p}^{k}(n)$-component of $\theta$. Then, by the proof of Lemma 3.4 (iii), the structure equation;

$$
\mathrm{d} \theta+\frac{1}{2}[\theta \wedge \theta]=\mathrm{d} \theta+\left[\theta \wedge \theta_{V}\right]=0
$$

of $\int^{k}(n)$ is expressed, in terms of the above 1-forms, as follows;

$$
\mathrm{d} \hat{\pi}=\sum_{i=1}^{n} \hat{\omega}_{i} \wedge \hat{\pi}_{i},
$$




$$
\begin{aligned}
& \mathrm{d} \hat{\pi}_{I}=\sum_{j=1}^{n} \hat{\omega}_{j} \wedge \hat{\pi}_{I, j}\left(I \in \Sigma_{k-1}\right), \\
& \mathrm{d} \hat{\omega}_{i}=\mathrm{d} \hat{\pi}_{J}=0\left(i=1, \cdots, n, J \in S_{k}\right),
\end{aligned}
$$

where we use the convention that $\hat{\pi}_{I}$ is defined for any $I \in \bigcup_{r=1}^{k} M_{r}$ such that $\hat{\pi}_{I}$ is symmetric with respect to $I$. These being remarked, let $x$ be any point of $K$. Since $C$ is a regular differential system of type $\widetilde{C}^{k}(n)$, the argument in $\left[19\right.$, p. 22] and the proof of Proposition 4.2 [19] show that there exists a $\complement^{k}(n)-$ valued 1-form $\xi=\sum_{p=-1}^{-(k+1)} \xi_{p}$ defined on a neighborhood $U$ of $x$, which defines an absolute parallelism on $U$, such that $\partial^{(-p-1)} C$ is defined on $U$ by $\xi_{r}(r<p)$ and that the following equalities hold;

$$
\mathrm{d} \xi_{p}+\left[\xi_{p+1} \wedge \xi_{V}\right] \equiv 0\left(\bmod \xi_{r} \wedge \xi_{s}(r+s<p)\right),
$$

where $[$,$] is the bracket product of \mathbb{C}^{k}(n)$. Then, expressing $\xi$ with respect to the above basis of $\mathcal{C}^{k}(n)$, we obtain 1 -forms $\pi, \omega_{1}, \cdots, \omega_{n}$ and $\pi_{I}\left(I \in \Sigma_{k}\right)$ defined on $U$, which form a basis of $T_{y}^{*}(K)$ at each $y \in U$, such that $C^{r+1}=$ $\partial^{(k-r-1)} C$ is defined on $U$ by $\pi$ and $\pi_{I}\left(I \in \Sigma_{r}\right)$ for $r=0, \cdots, k-1$ and that the following equalities hold on $U(r=1, \cdots, k-1)$;

$$
\begin{aligned}
\mathrm{d} \pi \equiv \sum_{i=1}^{n} \omega_{i} \wedge \pi_{i} & \left(\bmod \pi, \pi_{J_{1}} \wedge \pi_{J_{2}}\left(\left(J_{1}, J_{2}\right) \in \hat{S}(0)\right)\right), \\
\mathrm{d} \pi_{I} \equiv \sum_{j=1}^{n} \omega_{j} \wedge \pi_{I, j} & \left(\bmod \pi, \pi_{J}\left(J \in \Sigma_{r}\right), \pi_{J_{1}} \wedge \pi_{J_{2}}\left(\left(J_{1}, J_{2}\right) \in \hat{S}(r)\right)\right),
\end{aligned}
$$

for $I \in S_{r}$, where $\left(J_{1}, J_{2}\right) \in \hat{S}(r)$ means that $J_{1} \in S_{r_{1}}, J_{2} \in S_{r_{2}}, r_{1}+r_{2}<r+k+1$ and $r_{1}, r_{2}>r$. In particular $C^{1}=\partial^{(k-1)} C$ is defined on $U$ by $\pi$ and $C^{2}=\partial^{(k-2)} C$ is defined by $\pi_{1}, \cdots, \pi_{n}$ and $\pi$.

Assume that $C^{1}=\partial C^{2}$. Then, by Lemma 1.1, we have

$$
\mathrm{d} \pi \equiv 0 \quad\left(\bmod \pi, \pi_{1}, \cdots, \pi_{n}\right) .
$$

Hence, by (6.1), we get

$$
\mathrm{d} \pi \equiv \sum_{i=1}^{n} \omega_{i} \wedge \pi_{i} \quad\left(\bmod \pi, \pi_{i} \wedge \pi_{J}\left(i=1, \cdots, n, J \in \Sigma_{k-1}\right)\right) .
$$

Then there exist 1 -forms $\tilde{\omega}_{1}, \cdots, \tilde{\omega}_{n}$ defined on $U$ such that

$$
\begin{aligned}
\tilde{\omega}_{i} & \equiv \omega_{i} \quad\left(\bmod \pi, \pi_{J}\left(J \in \Sigma_{k-1}\right)\right) \text { for } i=1, \cdots, n, \\
\mathrm{~d} \pi & \equiv \sum_{i=1}^{n} \tilde{\omega}_{i} \wedge \pi_{i} \quad(\bmod \pi) .
\end{aligned}
$$

Furthermore, by the choice of $\tilde{\omega}_{1}, \cdots, \tilde{\omega}_{n}$, we have

$$
d \pi_{I} \equiv \sum_{j=1}^{n} \check{\omega}_{j} \wedge \pi_{I, j} \quad\left(\bmod \pi, \pi_{J}\left(J \in \Sigma_{r}\right), \pi_{J_{1}} \wedge \pi_{J_{2}}\left(\left(J_{1}, J_{2}\right) \in \hat{S}(r)\right)\right)
$$


for $I \in S_{r}$. Now we claim

$$
\mathrm{d} \pi_{I} \equiv \sum_{j=1}^{n} \tilde{\omega}_{j} \wedge \pi_{I, j} \quad\left(\bmod \pi, \pi_{J}\left(J \in \Sigma_{r}\right)\right) \text { for } I \in S_{r} .
$$

(One should note that $(6.4)_{p+k+1}$ is equivalent to the following:

$$
\mathrm{d} \xi_{p}+\left[\xi_{p+1} \wedge \tilde{\xi}_{V}\right] \equiv 0 \quad\left(\bmod \xi_{r}(r \leqq p)\right)
$$

where $\tilde{\xi}_{V}=\sum_{i=1}^{n} \tilde{\omega}_{i} \cdot e_{i}$.) We will prove this claim by induction on $r$. For $r=0$, we have (6.2). Assume that (6.4) holds for $m \leqq r$. Let $I$ be any element of $S_{r}$. Here we understand that $S_{0}$ is an empty set. By (6.3), there exist 2 -forms $\Pi_{I, i}=\sum_{\left(J_{1}, J_{2}\right) \in S(r+1)} a_{J_{1}, J_{2}}^{I, i} \pi_{J_{1}} \wedge \pi_{J_{2}}(i=1, \cdots, n)$ on $U$ such that

$$
\mathrm{d} \pi_{I, i} \equiv \sum_{j=1}^{n} \tilde{\omega}_{j} \pi_{I, i, j}+\Pi_{I, i} \quad\left(\bmod \pi, \pi_{J}\left(J \in \Sigma_{r+1}\right)\right) .
$$

On the other hand, from $(6.4)_{r}$, it follows that

$$
\sum_{i=1}^{n} \tilde{\omega}_{i} \wedge \mathrm{d} \pi_{I, i} \equiv 0 \quad\left(\bmod \pi, \pi_{J}\left(J \in \Sigma_{r+1}\right)\right) \text { for } I \in S_{r}
$$

(One should note that (6.6) is equivalent to the following:

$$
\left.\left[\mathrm{d} \xi_{p} \wedge \tilde{\xi}_{V}\right] \equiv 0 \quad\left(\bmod \xi_{r}(r \leqq p)\right) .\right)
$$

Hence, substituting (6.5) into (6.6), by the Jacobi identity of $\varsigma^{k}(n)$, we get

$$
\sum_{i=1}^{n} \tilde{\omega}_{i} \wedge \Pi_{I, i} \equiv 0 \quad\left(\bmod \pi, \pi_{J}\left(J \in \Sigma_{r+1}\right)\right) \text { for } I \in S_{r}
$$

Therefore we obtain $\Pi_{I, i}=0$, whence $(6.4)_{r+1}$ holds.

From (6.2) and (6.4), it follows that $C^{r}=\partial C^{r+1}$ for $r=1, \cdots, k-1$ and that $C h\left(C^{r}\right)$ is defined on $U$ by $\tilde{\omega}_{1}, \cdots, \tilde{\omega}_{n}, \pi$ and $\pi_{I}\left(I \in \Sigma_{r}\right)$ for $r=1, \cdots, k$. Hence $C h\left(C^{r}\right)$ is a subbundle of $C^{r+1}$ of codimension $n$ for $r=1, \cdots, k-1$. Furthermore, it is easy to see that $C h\left(C^{k-1}\right)=C h\left(C^{1}\right) \cap C^{k}$ and $C h\left(C^{k}\right)=\{0\}$. Therefore, $(K, C)$ is a contact manifold of order $k$ of degree $n$ q.e.d.

Corollary 6.6. Let $C$ be a regular differential system on a manifold $K$ such that $\partial^{(\mu)} C=T(K)$ for some $\mu$. Then $(K, C)$ is a contant manifold of order 2 (resp. order 3 ) of degree $n$ if and only if $C$ is a regular differential system of type $\overleftarrow{\complement}^{2}(n)$ (resp. of type $\left.\mathfrak{C}^{3}(n)\right)$. Furthermore, $(K, C)$ is a contact manifold of order 4 of degree 1 if and only if $C$ is of type $\mathcal{S}^{4}(1)$.

Proof. Let $C$ be a regular differential system of type $\mathbb{C}^{k}(n)$. Notations being the same as in the proof of Theorem 6.5, let $I(k)$ denote the set of ordered pairs $\left(r_{1}, r_{2}\right)$ of positive integers such that $r_{1} \leqq r_{2}$ and $r_{1}+r_{2}<k+1$. 
Then we have $I(2)=\{(1,1)\}, I(3)=\{(1,1),(1,2)\}$ and $I(4)=\{(1,1),(1,2),(1,3)$, $(2,2)\}$. Hence, if $k=2,3$ or $(k, n)=(4,1)$, by $(6.1)$, we easily get

$$
\mathrm{d} \pi \equiv 0 \quad\left(\bmod \pi, \pi_{1}, \cdots, \pi_{n}\right),
$$

which implies that $C^{1}=\partial C^{2}$. Therefore the assertion follows from Theorem 6.5 . q.e.d.

Thus we have characterized contact manifolds of order 2 or 3 of degree $n$ solely by their graded algebras.

REMARK 6.7. (1) By the proof of Theorem 6.5 and Theorem 6.2, we have the following fact (cf. [6, p. 20]): Let $K$ be a manifold of dimension $\sum_{r=1}^{k} H_{r}+n+1$ and let $C$ be a differential system on $K$. Let $x$ be any point of $K$. Then we can find a coordinate system $\left(x_{1}, \cdots, x_{n}, z, p_{I}\right)\left(I \in \Sigma_{k}\right)$ of $K$ defined around $x$ such that $C$ is defined by the following 1-forms:

$$
\begin{aligned}
& \hat{\pi}=\mathrm{d} z-\sum_{i=1}^{n} p_{i} \mathrm{~d} x_{i}, \\
& \hat{\pi}_{I}=\mathrm{d} p_{I}-\sum_{j=1}^{n} p_{I, j} \mathrm{~d} x_{j}\left(I \in \sum_{k-1}\right),
\end{aligned}
$$

if (and only if) there exist 1-forms $\omega_{1}, \cdots, \omega_{n}, \pi$ and $\pi_{I}\left(I \in \Sigma_{k}\right)$ defined around $x$, which form a basis of 1 -forms around $x$, such that $C$ is defined by $\pi$ and $\pi_{I}$ $\left(I \in \Sigma_{k-1}\right)$ and that the following equalities hold:

$$
\begin{aligned}
\mathrm{d} \pi & \equiv \sum_{i=1}^{n} \omega_{i} \wedge \pi_{i} \quad(\bmod \pi), \\
\mathrm{d} \pi_{I} & \equiv \sum_{j=1}^{n} \omega_{j} \wedge \pi_{I, j} \quad\left(\bmod \pi, \pi_{J}\left(J \in \Sigma_{r}\right)\right)
\end{aligned}
$$

for $I \in S_{s}(r=1, \cdots, k=1)$. This fact follows essentially from the Darboux's theorem for $\pi$ (cf. the proof of Theorem 5.3). In other words we have only to integrate (i.e., to find a first integral of) completely integrable differential systems $n$ times to obtain coordinate functions $x_{1}, \cdots, x_{n}, z$ and $p_{I}\left(I \in \Sigma_{k}\right)$ around $x$.

(2) Let $\left(R ; D^{1}, \cdots, D^{k}\right)$ be a regularly involutive PD manifold of order $k$ of degree $n$ of type $\xi$. Then $\xi=\sum_{p=-1}^{-(k+1)} \xi_{p}$ is an involutive subalgebra of $\mathfrak{C}^{k}(n)=\mathfrak{C}^{k}(V, W)$ satisfying $\xi_{-(k+1)}=W$ and $\xi_{-k}=W \otimes V^{*}$. Here, by Proposition 3.7, we may assume that $\mathfrak{g}_{-1} \subset V$. Hence we have $\mathfrak{g}_{-1}=V \oplus \mathfrak{g}_{*}$ (direct sum), where $\mathfrak{g}_{*}=\mathfrak{g} \cap \mathfrak{F}(V, W)$. Let $\theta$ be the Maurer-Cartan form of $\mathfrak{g}$ with the structure equation;

$$
\mathrm{d} \theta+\frac{1}{2}[\theta \wedge \theta]=0
$$


Since $\left[\mathfrak{I}_{p}, \mathfrak{I}_{q}\right]=\left[\mathfrak{I}_{p}, \mathfrak{P}_{*}\right]=0$ if $p, q<-1$, we can rewrite the structure equation as follows:

$$
\mathrm{d} \theta+\left[\theta \wedge \theta_{V}\right]=0
$$

where $\theta_{V}$ is the $V$-component of $\theta$. Let $x$ be any point of $R$. Then, in the same manner as in Proposition 4.2 [19], there exists a $\xi$-valued 1-form $\xi=$ $\sum_{p=-1}^{-(k+1)} \xi_{p}$ defined on a neighborhood $U$ of $x$, which defines an absolute parallelism on $U$, such that $D^{p+k+1}$ is defined on $U$ by $\xi_{r}(r<p)$ for $p \leqq-1$ and that the following equalities hold;

$$
\mathrm{d} \xi_{p}+\left[\xi_{p+1} \wedge \xi_{V}\right] \equiv 0\left(\bmod \xi_{r}(r \leqq p), \xi_{p+1} \wedge \xi_{s}(p<s<-1)\right) .
$$

Furthermore, by $\mathfrak{I}_{-(k+1)}=W$ and $\mathfrak{g}_{-k}=W \otimes V^{*}$, in the same manner as in the proof of Theorem 6.5, we may assume, by replacing $\xi_{V}$, that

$$
\mathrm{d} \xi_{p}+\left[\xi_{p+1} \wedge \xi_{V}\right] \equiv 0 \quad\left(\bmod \xi_{r}(r \leqq p)\right) .
$$

These equations are precisely what E. Cartan called the structure formula of the involutive system $R$ in [5] and [6].

\section{§7. Monge characteristics and covariant systems}

7.1. Polar subspaces and characteristic ideal $\mathfrak{M}(\mathfrak{f}(x))$. Let $\left(R ; D^{1}, \cdots\right.$, $D^{k}$ ) be an involutive PD manifold of order $k$ of degree $n$. In this and the subsequent paragraphs, we fix a point $x$ of $R$. Let $\xi=\xi(x)$ be the symbol algebra at $x$. Set $V=V(x)\left(=\mathscr{g}_{-1}(x) / f(x)\right)$ and let $\kappa$ be the projection of $\mathfrak{g}_{-1}(x)$ onto $V$. Let us fix a basis $w_{0}$ of $\mathfrak{Z}_{-(k+1)}=\mathfrak{g}_{-(k+1)}(x)$ and identify $\mathfrak{g}_{-(k+1)} \otimes S^{r}\left(V^{*}\right)$ with $S^{r}\left(V^{*}\right) . \quad\left(S^{r}\left(V^{*}\right)\right)^{*}$ is naturally identified with $S^{r}(V)$ via the natural identification of $\left(\otimes^{r} V^{*}\right)^{*}$ with $\otimes^{r} V$. Let $\tau_{-1}=\tau_{-1}(x)$ be the fundamental identification of $\uparrow=\digamma(x)$ at $x$ (Proposition $5.9(1)$ ). Let $V_{r}$ be an $r$-dimensional subspace of $V$ and set

$$
\begin{aligned}
& \hat{\mathfrak{f}}=\tau_{-1}(\mathfrak{f}) \subset S^{k}\left(V^{*}\right), \\
& \hat{\mathfrak{f}}\left(V_{r}\right)=\left\{a \in \hat{\mathfrak{f}} \mid v-a=0 \text { for any } v \in V_{r}\right\} .
\end{aligned}
$$

Let $(\hat{\hat{f}})^{\perp}$ be the annihilator subspace of $\hat{\hat{\varphi}}$ in $S^{k}(V)$ and let $p \hat{f}$ denote the first prolongation of $\hat{\mathfrak{f}}$. For subspaces $A \subset S^{r}(V)$ and $B \subset S^{s}(V)$, let $A \otimes_{s} B$ be the linear subspace of $S^{r+s}(V)$ generated by the set of elements $a @ b$ for $a \in A$ and $b \in B$, where (O) denotes the symmetric product. Then we have easily

LEMMA 7.1. ( i ) $(p \hat{\mathrm{f}})^{\perp}=(\hat{\mathrm{f}})^{\perp} \otimes_{s} V$,

(ii) $\left(\hat{\mathfrak{f}}\left(V_{r}\right)\right)^{\perp}=(\hat{\mathfrak{f}})^{\perp}+V_{r} \otimes_{s} S^{k-1}(V)$.

Let $E_{r}$ be an $r$-dimensional integral element of $\left(R, D^{k}\right)$ at $x$ such that 
$E_{r} \cap f(x)=\{0\}$. Then the polar subspace (i.e., the space of solutions of the polar equation) $H\left(E_{r}\right)$ of $E_{r}$ is, by definition ([14, p. 47]), given by

$$
H\left(E_{r}\right)=\left\{X \in \mathfrak{Z}_{-1}(x) \mid\left[X, E_{r}\right]=0\right\} .
$$

LEMMA 7.2. $\tau_{-1}\left(H\left(E_{r}\right) \cap \mathfrak{f}\right)=\hat{\mathfrak{f}}\left(V_{r}\right)$, where $V_{r}=\kappa\left(E_{r}\right)$.

Proof. Take a point $w \in R^{(1)}(x)$ and let $\phi_{w}$ be the isomorphism of $g$ into $\mathfrak{C}^{k}\left(V, \mathfrak{g}_{-(k+1)}\right)$ given in the proof of Proposition 5.10 (3). Let $X \in \mathfrak{f}$ and $Y \in E_{r}$. Then

$$
\left.\phi_{w}([X, Y])=\left[\tau_{-1}(X), \kappa(Y)\right]=\kappa(Y)\right\lrcorner \tau_{-1}(X)
$$

Hence we get

$$
\tau_{-1}\left(H\left(E_{r}\right) \cap \mathfrak{f}\right)=\left\{a \in \hat{\mathfrak{f}} \mid v-a=0 \text { for any } v \in V_{r}\right\} .
$$

Thus we get the assertion.

q.e.d.

Set $S(V)=\sum_{k=0}^{\infty} S^{k}(V)$. Then $S(V)$ is the ring of polynomials on $V^{*}$. Let $\mathfrak{X}$ denote the maximal ideal of $S(V)$, i.e., $\mathfrak{X}=\sum_{k=1}^{\infty} S^{k}(V)$. Let $\Re$ be a homogeneous ideal of $S(V)$. We denote by $H(\Re)$ the smallest (homogeneous) ideal $\mathfrak{M}$ of $S(V)$ which contains $\mathfrak{N}$ and which possesses the property

$$
\mathfrak{X} z \subset \mathfrak{M} \text { implies } z \in \mathfrak{M}
$$

(see $\S 3[11])$. One should recall that $H(H(\Re))=H(\Re)$ and that an irredundant primary decomposition of $H(\Re)$ in $S(V)$ is obtained from that of $\Re$ by omitting the component which is $\mathfrak{X}$-primary (if it exists) ( $\$ 3$ [11]).

Let $A$ be a subspace of $S^{k}\left(V^{*}\right)$. We denote by $\mathfrak{R}(A)=\sum_{q=k}^{\infty} \Re_{q}(A)$ the (homogeneous) ideal of $S(V)$ generated by $A^{\perp}$ and set $\mathfrak{M}(A)=H(\Re(A))$. $\mathfrak{M}(A)=\sum_{q=0}^{\infty} \mathfrak{M}_{q}(A)$ is called the ideal associated with $A$. Then the characteristic ideal $\mathfrak{M}(\mathfrak{f}(x))$ of $\left(R ; D^{1}, \cdots, D^{k}\right)$ at $x$ is, by definition, the ideal of $S(V(x))$ associated with $\hat{\mathfrak{f}}=\tau_{-1}(\tilde{f}(x))$. The notion of the characteristic ideal is introduced by K. Kakie [11]. Now we recall

Lemia 7.3 (Lemma 5 [11]). Let $A$ be an involutive subspace of $S^{k}\left(V^{*}\right)$. Then $\mathfrak{M}_{q}(A)=\mathfrak{N}_{q}(A)$ for all $q \geqq k$, i.e., $\mathfrak{M}(A) \cap \mathfrak{X}^{k}=\mathfrak{N}(A)$. In particular, $A$ is of finite type, i.e., $p^{(r)} A=\{0\}$ for some $r$, if and only if $A=\{0\}$.

Then we have

Proposition 7.4. Let $A$ be an involutive subspace of $S^{k}\left(V^{*}\right)$ and let $V_{r}$ be an $r$-dimensional subspace of $V$. Then

(1) $A^{\perp} \supset V_{r} \otimes_{s} S^{k-1}(V)$ if and only if $\mathfrak{M}(A) \supset V_{r}$.

(2) $A^{\perp} \subset V_{s} \otimes_{s} S^{k-1}(V)$ if and only if $\mathfrak{M}(A) \subset \mathfrak{S}\left(V_{r}\right)$, where $\mathfrak{S}\left(V_{r}\right)$ denotes the (prime) ideal of $S(V)$ generated by $V_{r} \subset S^{1}(V)$. 
Proof. Clearly the ideal generated by $V_{r} \otimes_{s} S^{k-1}(V)$ is given by $\mathfrak{\Im}\left(V_{r}\right)$ $\cap \mathfrak{X}^{k}$. Hence $A^{\perp} \supset V_{r} \otimes_{s} S^{k-1}(V)$ if and only if $\mathfrak{N}(A) \supset \mathfrak{S}\left(V_{r}\right) \cap \mathfrak{X}^{k}$. On the other hand, by Lemma 7.3, we have $\mathfrak{R}(A)=\mathfrak{M}(A) \cap \mathfrak{X}^{k}$. From $H\left(\mathfrak{s}\left(V_{r}\right) \cap \mathfrak{X}^{k}\right)=$ $\mathfrak{I}\left(V_{r}\right)$ and $H\left(\mathfrak{M}(A) \cap \mathfrak{X}^{k}\right)=\mathfrak{M}(A)$, it follows that $A^{\perp} \supset V_{r} \otimes_{s} S^{k-1}(V)$ if and only if $\mathfrak{M}(A) \supset \mathfrak{J}\left(V_{r}\right)$. Thus we get (1). (2) can be shown similarly. q.e.d.

REMARK 7.5. The above consideration is limited to the case when $\operatorname{dim} W$ $=1$. More generally, if $\operatorname{dim} W>1$, we have the following fact: Set $L=$ $\sum_{k=0}^{\infty} W^{*} \otimes S^{k}(V)$. Let $A$ be an involutive subspace of $W \otimes S^{k}\left(V^{*}\right)$ and let $M(A)$ be the module associated with $A\left(\S 3\right.$ [11]). Let $V_{r}$ be an $r$-dimensional subspace of $V$. We set $V_{r} L_{k-1}=W^{*} \otimes V_{r} \otimes_{s} S^{k-1}(V)$ and $V_{r} L=\sum_{k=0}^{\infty} W^{*} \otimes V_{r}$ $\otimes_{s} S^{k}(V)$. Then, similarly as in Proposition 7.4, we have

(1) $A^{\perp} \supset V_{r} L_{k-1}$ if and only if $M(A) \supset V_{r} L$.

(2) $A^{\perp} \subset V_{r} L_{k-1}$ if and only if $M(A) \subset V_{r} L$.

Furthermore, let Ann $(L / M(A))$ be the annihilator of $M(A)$ in $L$, i.e., Ann $(L / M(A))=\{a \in S(V) \mid a L \subset M(A)\}$. Then we have

(1) $A^{\perp} \supset V_{r} L_{k-1}$ if and only if $\operatorname{Ann}(L / M(A)) \supset V_{r}$.

(2) $A^{\perp} \subset V_{r} L_{k-1}$ implies $\operatorname{Ann}(L / M(A)) \subset S\left(V_{r}\right)$.

The converse of (2)' does not hold in general.

7.2. Characteristic elements. Let $E_{r}$ be an $r$-dimensional integral element of $\left(R, D^{k}\right)$ at $x$ such that there exists a point $w=E_{n}$ of $R^{(1)}(x)$ satisfying $E_{r} \subset E_{n}$. Then $E_{n} \subset H\left(E_{r}\right)$. Hence, by Lemma 7.2 , we have $\operatorname{dim} H\left(E_{r}\right)=$ $\operatorname{dim} \hat{\hat{f}}\left(V_{r}\right)+n$, where $V_{r}=\kappa\left(E_{r}\right)\left(\right.$ cf. Lemma 1 [15, p. 70]). Set $g_{r}=\operatorname{Min}\left\{\operatorname{dim} \hat{\mathfrak{f}}\left(V_{r}\right) \mid\right.$ $V_{r}: r$-dimensional subspace of $\left.V\right\}$. Then a generic (or ordinary) integral element $E_{r}$ satisfies $\operatorname{dim} H\left(E_{r}\right)=n+g_{r}$. In general $E_{r}$ is called a singular integral element if $\operatorname{dim} H\left(E_{r}\right)>n+g_{r}$. In the following, for a fixed integer $r(1 \leqq r \leqq n)$, we will seek an integral element $E_{r}$ such that $\operatorname{dim} H\left(E_{r}\right)$ attains the (possible) maximum value.

Let $E_{r}$ be an $r$-dimensional integral element of $\left(R, D^{k}\right)$ at $x$ such that $E_{r} \cap \tilde{f}(x)=\{0\}$, i.e., $\operatorname{dim} V_{r}=r$, where $V_{r}=\kappa\left(E_{r}\right)$. Let $\alpha=\alpha(R)(0 \leqq \alpha \leqq n-1)$ denote the unique integer satisfying ${ }_{n-\alpha-1} H_{k}<\operatorname{dim} f(x) \leqq_{n-\alpha} H_{k}$. First we have

LEMMA 7.6. Let $\mathfrak{M}(\mathfrak{f}(x))$ be the characteristic ideal at $x$.

(i) If $r \leqq \alpha(R)$, then $\operatorname{dim} H\left(E_{r}\right) \cap \uparrow(x) \leqq \operatorname{dim} \tilde{\uparrow}(x)$. The equality holds if and only if $V_{r} \subset \mathfrak{M}(\mathfrak{f}(x))$.

(ii) If $\alpha(R)<r<n$, then $\operatorname{dim} H\left(E_{r}\right) \cap \tilde{f}(x) \leqq{ }_{n-r} H_{k}(<\operatorname{dim} f(x))$. The equality holds if and only if $\mathfrak{\Im}\left(V_{r}\right) \supset \mathfrak{M}(\mathfrak{f}(x))$.

(iii) If $r=n$, then $H\left(E_{n}\right)=E_{n}$. 
Proof. Let $V_{r}^{\perp}$ denote the annihilator subspace of $V_{r}$ in $V^{*}$. Then, by Lemma 7.1 (ii) and Lemma 7.2, we have

$$
\tau_{-1}\left(H\left(E_{r}\right) \cap \mathfrak{f}\right)=\hat{\mathfrak{f}} \cap S^{k}\left(V_{r}^{\perp}\right),
$$

where $S^{k}\left(V_{r}^{\perp}\right)$ is naturally identified with the subspace of $S^{k}\left(V^{*}\right)$ consisting of elements $a \in S^{k}\left(V^{*}\right)$ such that $v \_a=0$ for any $v \in V_{r}$. Clearly $\operatorname{dim} S^{k}\left(V_{\frac{\perp}{r}}^{\perp}\right.$ $={ }_{n-r} H_{k}$. Hence we get

$$
\operatorname{dim} H\left(E_{r}\right) \cap \mathfrak{f}(x) \leqq \lambda_{r},
$$

where $\lambda_{r}=\operatorname{Min}\left\{\operatorname{dim} f(x),{ }_{n-r} H_{k}\right\}$.

(i) $r \leqq \alpha(R)$ implies $\lambda_{r}=\operatorname{dim} f(x)$. Then the equality holds if and only if $\tau_{-1}\left(H\left(F_{r}\right) \cap \hat{f}\right)=\hat{\mathfrak{f}}$, i.e., $(\hat{\mathfrak{f}})^{\perp} \supset V_{r} \otimes_{s} S^{k-1}(V)$. Hence the assertion follows from Proposition 7.4 (1).

(ii) The assertion follows from Proposition 7.4 (2) similarly as in (i).

(iii) $\lambda_{n}=0$. Hence $H\left(E_{n}\right) \cap f(x)=\{0\}$, which implies $\operatorname{dim} H\left(E_{n}\right) \leqq n$. On the other hand we have $E_{n} \subset H\left(E_{n}\right)$. Thus we get $H\left(E_{n}\right)=E_{n}$.

Now we have

Theorem 7.7. Let $\left(R ; D^{1}, \cdots, D^{k}\right)$ be an involutive $P D$ manifold of order $k$ of degree $n$. Let $E_{r}$ be an r-dimensional integral element of $\left(R, D^{k}\right)$ at $x$ such that $\operatorname{dim} V_{r}=r$, where $V_{r}=\kappa\left(E_{r}\right)$. Let $\mathfrak{M}(\mathfrak{f}(x))$ be the characteristic ideal at $x$. Then

(1) If $r \leqq \alpha(R)$, then $\operatorname{dim} H\left(E_{r}\right) \leqq \operatorname{dim} D^{k}(x)$. The equality holds if and only if $V_{r} \subset \mathfrak{M}(\mathfrak{f}(x))$ and there exists a point $w$ of $R^{(1)}(x)$ such that $E_{r} \subset w$. Furthermore, if the equality holds, $E_{r} \subset w$ for any $w \in R^{(1)}(x)$.

(2) If $r>\alpha(R)$, then $\operatorname{dim} H\left(E_{r}\right) \leqq{ }_{n-r} H_{k}+n\left(<\operatorname{dim} D^{k}(x)\right)$. The equality holds if and only if $\mathfrak{s}\left(V_{r}\right) \supset \mathfrak{M}(\mathfrak{f}(x))$ and there exists a point $w$ of $R^{(1)}(x)$ such that $E_{r} \subset w$.

Proor. The inequalities follow immediately from Lemma 7.6. It remains to show the equality conditions.

(1) The if part follows immediately from Lemma 7.6. If $\operatorname{dim} H\left(E_{r}\right)=$ $\operatorname{dim} D^{k}(x)$, we have $H\left(E_{r}\right)=D^{k}(x)$. Hence, by Lemma 7.6 (i), we get $V_{r} \subset$ $\mathfrak{M}(f(x))$. Furthermore, by the definition of polar subspaces, we have

$$
\left[X, \mathscr{P}_{-1}(x)\right]=0 \quad \text { for any } X \in E_{r} \text {. }
$$

Let $w=E_{n}$ be any point of $R^{(1)}(x)$. Since $\left[X, E_{n}\right]=0$ for any $X \in E_{r}$, we get $E_{r} \subset H\left(E_{n}\right)$. Then, by Lemma 7.6 (iii), we obtain $E_{r} \subset E_{n}$.

(2) The if part follows immediately from Lemma 7.6. If $\operatorname{dim} H\left(E_{r}\right)=$ ${ }_{n-r} H_{k}+n$, we must have $\operatorname{dim} H\left(E_{r}\right) \cap \mathfrak{f}(x)={ }_{n-r} H_{k}$. Then, by the proof of Lemma 7.6, we get $\mathfrak{S}\left(V_{r}\right) \supset \mathfrak{M}(\mathfrak{f})$ and $\tau_{-1}\left(H\left(E_{r}\right) \cap \mathfrak{f}\right)=S^{k}\left(V_{r}^{\perp}\right)$. Furthermore, 
since $\operatorname{dim} H\left(E_{r}\right) / H\left(E_{r}\right) \cap \mathfrak{f}=n$, there exists an $n$-dimensional subspace $E$ of $H\left(E_{r}\right)$ such that $\kappa(E)=V$ and $E_{r} \subset E$. Then, setting $V_{0}=E$ in the proof of Proposition 5.7 (2), we get an isomorphism $\phi_{E}$ of $\mathfrak{g}$ into $\mathcal{C}^{k}\left(V, \mathfrak{Z}_{-(k+1)}\right)$. Recall that $\phi_{E}$ is defined by

$$
\begin{aligned}
& \left.\phi_{E}\right|_{\beta_{-(k+1)}}=i d_{\vec{\beta} \rightarrow(k+1)},\left.\quad \phi_{E}\right|_{z_{p}}=\tau_{p}(-k \leqq p \leqq-2), \\
& \left.\phi_{E}\right|_{\mathrm{f}}=\left.\tau_{-1} \quad \phi_{E}\right|_{E}=\kappa+\hat{\rho} \cdot \kappa,
\end{aligned}
$$

where $\hat{\rho} ; V \rightarrow S^{k}\left(V^{*}\right)$ is defined by (5.1). Take a basis $X_{1}, \cdots, X_{n}$ of $E$ such that the vectors $X_{1}, \cdots, X_{r}$ span $E_{r}$. Set $\kappa\left(X_{i}\right)=e_{i}$. Then $e_{i}, \cdots, e_{n}$ is a basis of $V$. By $\left[X_{i}, E\right]=0(i=1, \cdots, r)$ and (5.1), we get

$$
\hat{\rho}\left(e_{i}\right)=0 \quad \text { for } i=1, \cdots, r,
$$

which implies $\phi_{E}\left(E_{r}\right) \subset V$. Furthermore, by (5.2), we have

$$
\left.\tau_{-2}\left(\left[X_{i}, X_{\alpha}\right]\right)=e_{\alpha}\right\lrcorner \hat{\rho}\left(e_{i}\right)-e_{i}-\hat{\rho}\left(e_{\alpha}\right)=0
$$

for $i=1, \cdots, r$ and $\alpha=r+1, \cdots, n$. Hence we obtain $e_{i}-\hat{\rho}\left(e_{\alpha}\right)=0(1 \leqq i \leqq r$, $r+1 \leqq \alpha \leqq n)$, i.e.,

$$
\hat{\rho}\left(e_{\alpha}\right) \in S^{k}\left(V_{r}^{\perp}\right) \quad \text { for } \alpha=r+1, \cdots, n .
$$

Then, from $S^{k}\left(V_{r}^{\perp}\right)=\tau_{-1}\left(H\left(E_{r}\right) \cap \mathfrak{f}\right) \subset \tau_{-1}(\mathfrak{f})=\phi_{E}(\mathfrak{f})$, we conclude $V \subset \phi_{E}\left(\mathfrak{G}_{--1}\right)$. Thus we find a point $w=\phi_{\phi}^{-1}(V)$ of $R^{(1)}(x)$ such that $E_{r} \subset w$. q.e.d.

Corollary 7.8 (cf. [12]). Let $\left(R ; D^{1}, \cdots, D^{k}\right)$ be an involutive PD manifold of order $k$ of degree $n$. Let $\mathfrak{M}(f(x))$ be the characteristic ideal at $x \in R$. Then $\kappa\left(C h\left(D^{k}\right)(x)\right)=\mathfrak{M}(f(x)) \cap V(x)$.

Proof. Let $E_{r}$ be an $r$-dimensional integral element of $\left(R, D^{k}\right)$ at $x$. Then, as is well known, $E_{r} \subset C h\left(D^{k}\right)(x)$ if and only if $H\left(E_{r}\right)=D^{k}(x)$. On the other hand we have $f(x) \cap C h\left(D^{k}\right)(x)=\{0\}$. Hence the assertion follows immediately from Theorem 7.7 (1).

q.e.d.

Let $E_{r}$ be an $r$-dimensional integral element of $\left(R, D^{k}\right)$ at $x$ such that $E_{r} \cap f(x)=\{0\} . \quad$ In view of Theorem 7.7 (2), we call $E_{r}$ a Monge element if it satisfies $\operatorname{dim} H\left(E_{r}\right)={ }_{n-r} H_{k}+n$. For a Monge element $E_{r}$, there exists a point $w=E_{n}$ of $R^{(1)}(x)$ such that $E_{r} \subset E_{n}$. Let $E_{s}$ be a subspace of $E_{n}$ such that $E_{r} \subset E_{s}$. Then, from Theorem 7.7 (2), it follows that $E_{s}$ is also a Monge element. This being remarked, we give the following definition.

Definition 7.9 (cf. [6], [10]). Let $E_{r}(1 \leqq r \leqq n-1)$ be an $r$-dimensional integral element of $\left(R, D^{k}\right)$ at $x$ such that $E_{r} \cap f(x)=\{0\}$. Then $E_{r}$ is called $a$ Monge characteristics element if it is a minimal Monge element, i.e., if $E_{r}$ satisfies $\operatorname{dim} H\left(E_{r}\right)={ }_{n-r} H_{k}+n$ and no $s$-dimensional subspace $E_{s}$ of $E_{r}$ satisfies 
$\operatorname{dim} H\left(E_{s}\right)={ }_{n-s} H_{k}+n$ for $s<r$.

Let $\left\{\mathfrak{P}_{1}, \cdots, \mathfrak{P}_{m}\right\}$ be the set of isolated prime ideals of $\mathfrak{M}(\mathfrak{f}(x))$, i.e., $r(\mathfrak{M}(\mathfrak{f}(x)))=\bigcap_{i=1}^{m} \mathfrak{P}_{i}$ (irredundant prime decomposition), where $r(\mathfrak{M}(\mathfrak{f}(x)))$ is the radical of $\mathfrak{M}(f(x))$. Let $E_{r}$ be an $r$-dimensional integral element of $\left(R, D^{k}\right)$ at $x$ which is contained in a point of $R^{(1)}(x)$. Then, from Theorem 7.7 (2), it follows that $E_{r}$ is a Monge element if and only if $\mathfrak{P}_{i} \subset \mathfrak{S}\left(V_{r}\right)$ for some $i$, where $V_{r}=\kappa\left(E_{r}\right)$. Furthermore, assume that $\mathfrak{P}_{i}$ is generated by elements of homogeneous degree 1, i.e., $\mathfrak{P}_{i}=\mathfrak{S}\left(V_{r}^{i}\right)$ for some $r$-dimensional subspace $V_{r}^{i}$ of $V$. Then $E_{r}$ is a Monge characteristic element if $\kappa\left(E_{r}\right)=V_{r}^{i}$. In this case $E_{r}$ is called a Monge characteristic element corresponding to $\mathfrak{P}_{i}$.

REMARK 7.10. In view of Remark 7.5, Theorem 7.7 (1), and hence Corollary 7.8, can be generalized accordingly to involutive systems with more than one unknown functions (cf. [12]). On the other hand, it seems to us that the concept of Monge characteristic elements of involutive systems with more than one unknown functions needs further geometric investigations.

7.3. Covariant systems of regularly involutive $P D$ manifolds. Let $(R$; $\left.D^{1}, \cdots, D^{k}\right)$ be a regularly involutive PD manifold of order $k$ of degree $n$ of type $\mathfrak{S}$. Then $\mathfrak{Z}=\sum_{p=-1}^{-(k+1)} \mathfrak{g}_{p}$ is an involutive subalgebra of $\mathfrak{C}^{k}(n)=\mathfrak{S}^{k}(V, W)$ satisfying $\xi_{-(k+1)}=W$ and $\xi_{-k}=W \otimes V^{*}$. Here, by Proposition 3.7, we may assume that $\mathfrak{g}_{-1} \supset V$. Let $A(\mathfrak{g})$ be the group of graded Lie algebra automorphisms of $\xi$. Then, by Corollary 5.8 , we have

$$
\begin{aligned}
& A(\mathfrak{I})=\left\{\phi \in A^{k}(V, W) \mid \phi(\mathfrak{I})=\mathfrak{g}\right\}, \\
& A(\mathfrak{g})=G(\mathfrak{g}) \cdot N(\mathfrak{g}) \text { (semi-direct product), }
\end{aligned}
$$

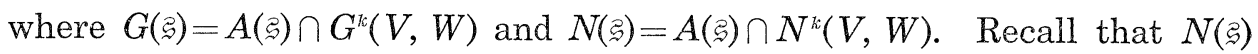
is canonically isomorphic with the vector group $p \xi_{*}$ and $G(\xi)=\{\phi \in A(\mathfrak{g}) \mid \phi(V)$ $=V\}$, where $\mathfrak{g}_{*}=\mathfrak{g} \cap \mathfrak{F}(V, W)$. Furthermore, we define the subgroup $G^{\prime}(\mathfrak{g})$ of $G L(V)$ by setting

$$
G^{\prime}(\mathfrak{g})=\left\{a \in G L(V) \mid \chi^{p+k+1}(a)\left(\mathfrak{g}_{p}\right)=\mathfrak{g}_{p}(-k+1 \leqq p \leqq-2), \chi^{k}(a)\left(\mathfrak{g}_{*}\right)=\mathfrak{g}_{*}\right\},
$$

where $\chi^{r}(a)=\chi^{r}\left(a, i d_{W}\right)(2 \leqq r \leqq k)$. Then, from $\operatorname{dim} W=1$, we see that $\chi\left(G^{\prime}(\mathfrak{g}) \times\right.$ $G L(W))=G(\xi)$, where $\chi$ is the isomorphism of $G L(V) \times G L(W)$ onto $G^{k}(V, W)$ given in Proposition 3.7. Let $\xi^{(1)}$ be the first prolongation of $\xi$ (cf. Proposition 5.16). Then one should note that $G^{\prime}(\mathfrak{I})=G^{\prime}\left(\mathfrak{g}^{(1)}\right)$.

Let $E$ be a $G($ ())-invariant subspace of $V$, i.e., $\phi(E)=E$ for every $\phi \in G($ ()). Obviously $E$ is $G(\xi)$-invariant if and only if it is $G^{\prime}(\xi)$-invariant. Now we will construct covariant systems $N(E)$ and $M(E)$ of $\left(R ; D^{1}, \cdots, D^{k}\right)$ as follows.

( I) Covariant system $N(E)$. Let $E^{\perp}$ be the annihilator subspace of $E$. Then $n(E)=W \otimes E^{\perp}$ is an $A(\xi)$-invariant subspace of $\xi_{-k}=W \otimes V^{*}$. Let $x$ be 
any point of $R$ and let $z(x)$ be the symbol algebra at $x$. Take a graded Lie algebra isomorphism $\phi$ of $\xi(x)$ onto $\xi$. Let $n(E)(x)$ denote the linear subspace of $\mathfrak{I}_{-k}(x)$ defined by

$$
\mathfrak{n}(E)(x)=\phi^{-1}(\mathfrak{n}(E)) .
$$

Then, since $\mathfrak{n}(E)$ is $A(\mathfrak{g})$-invariant, it follows from Proposition 5.10 (2) that $\mathfrak{n}(E)(x)$ is well-defined, i.e., the above definition is independent of the choice of $\phi$. Furthermore, $\mathfrak{n}(E)(x)$ is an $A(\xi(x))$-invariant subspace of $\xi(x)$, where $A(\xi(x))$ is the group of graded Lie algebra automorphisms of $\xi(x)$. Let $\kappa_{-k}$ be the projection of $D^{1}(x)$ onto $g_{-k}(x)=D^{1}(x) / D^{2}(x)$. Now we define the linear subspace $N(E)(x)$ of $D^{1}(x)$ by setting

$$
N(E)(x)=\left(\kappa_{-1}\right)^{-1}(\mathfrak{n}(E)(x)) .
$$

Then it follows that the assignment $x \mapsto N(E)(x)$ defines a subbundle $N(E)$ of $D^{1}$ (cf. Remark $6.7(2)$ ). $N(E)$ is called the first order covariant system corresponding to $E$.

(II) Covariant system $M(E)$. Let $m(E)$ denote the linear subspace of $\xi_{-1}$ spanned by linear subspaces $\phi(E), \phi \in A(\mathfrak{g})$, i.e.,

$$
m(E)=\left\langle\left\{\dot{\phi}(E) \subset \mathfrak{g}_{-1} \mid \phi \in A(\mathfrak{g})\right\}\right\rangle .
$$

Then we have

LEMma 7.11. $\mathfrak{m}(E)=E \oplus c(E)($ direct sum $)$, where

$$
\mathfrak{c}(E)=\left\langle\left\{\rho(E) \mid \rho \in p \mathfrak{s}_{*}\right\}\right\rangle \subset \mathfrak{g}_{*} .
$$

Proof. Let $A_{\rho} \in N^{k}(V, W)$. Then $A_{\rho} \in N(\mathfrak{g})$ if and only if $\rho \in p \mathfrak{g}_{*}$ (Corollary $5.8(1)$ ). Since $A_{\rho}(X)=X+\rho(X)$ for $X \in E$, we have

$$
E \oplus \mathfrak{c}(E)=\left\langle\left\{A_{\rho}(E) \subset \mathfrak{g}_{-1} \mid A_{\rho} \in N(\xi)\right\}\right\rangle .
$$

Let $\phi \in A(\mathfrak{\xi})$. Since $A(\mathfrak{\xi})$ is a semi-direct product of $G(\xi)$ and $N(\xi)$, there exist $\phi^{\prime} \in G(\mathfrak{I})$ and $A_{\rho} \in N(\mathfrak{I})$ such that $\phi=A_{\rho} \cdot \phi^{\prime}$. Then, from $\phi^{\prime}(E)=E$, we get $\phi(E)$ $=A_{\rho}(E)$. Therefore, we obtain $\mathfrak{m}(E)=E \oplus \mathrm{c}(E)$.

q.e.d.

Obviously $\mathfrak{m}(E)$ is an $A(\mathfrak{g})$-invariant subspace of $\mathfrak{z}_{-1}$. Let $x$ be any point of $R$ and let $\xi(x)$ be the symbol algebra at $x$. Take a graded Lie algebra isomorphism $\phi$ of $\mathfrak{z}(x)$ onto $\mathfrak{s}$. Let $M(E)(x)$ denote the linear subspace of $\mathfrak{g}_{-1}(x)$ $=D^{k}(x)$ defined by

$$
M(E)(x)=\phi^{-1}(\mathfrak{m}(E)) .
$$

Since $\mathfrak{m}(E)$ is $A(\xi)$-invariant, $M(E)(x)$ is well-defined. Furthermore, it follows that the assignment $x \mapsto M(E)(x)$ defines a subbundle $M(E)$ of $D^{k}$ (cf. Remark 
6.7 (2)). $M(E)$ is called the $k$-th order covariant system corresponding to $E$. Let $E(x)$ be the linear subspace of $V(x)=\mathfrak{Z}_{-1}(x) / \mathfrak{f}(x)$ defined by $E(x)=\hat{\phi}^{-1}(E)$, where $\hat{\phi}$ is the linear isomorphism of $V(x)$ onto $V$ satisfying $q \cdot \phi_{-1}=\hat{\phi} \cdot \kappa$ and $q$ is the projection of $\mathfrak{g}_{-1}$ onto $V$ corresponding to $\mathfrak{g}_{-1}=V \oplus \mathfrak{s}_{*}$. Then $E(x)$ is well-defined and one should note that $M(E)(x)$ is the linear subspace of $D^{k}(x)$ spanned by linear subspaces $E_{w}$ of $w$ satisfying $\kappa\left(E_{w}\right)=E(x)$ for all $w \in R^{(1)}(x)$ (cf. Proposition 3 [11]).

Summarizing the discussion above, we easily obtain

Proposition 7.12. Let $\left(R ; D^{1}, \cdots, D^{k}\right)$ and $\left(\hat{R} ; \hat{D}^{1}, \cdots, \hat{D}^{k}\right)$ be regularly involutive $P D$ manifolds of order $k$ of degree $n$ of type $\mathfrak{s}$. Let $E$ be a G(:)invariant subspace of $V$. Let $N(E)($ resp. $\hat{N}(E))$ and $M(E)($ resp. $\hat{M}(E))$ be the first and $k$-th order covariant systems of $R$ (resp. $\hat{R}$ ) corresponding to $E$ respectively. Then an isomorphism $\psi$ of $\left(R ; D^{1}, \cdots, D^{k}\right)$ onto $\left(\hat{R} ; \hat{D}^{1}, \cdots, \hat{D}^{k}\right)$ satisfies $\psi_{*}(N(E))=\hat{N}(E)$ and $\psi_{*}(M(E))=\hat{M}(E)$.

Let $\mathfrak{M}(\mathfrak{s})$ be the ideal associated with $\mathfrak{g}_{*}$. Let $\mathfrak{P}_{1}, \cdots, \mathfrak{P}_{\nu}$ be the associated prime ideals of $\mathfrak{M}(\mathfrak{s})$. Let $G^{0}(\mathfrak{s})$ and $A^{0}(\mathfrak{I})$ denote the identity components of $G(\xi)$ and $A(\xi)$ respectively. Then, by the uniqueness of the associated prime ideals, $E_{i}=\mathfrak{P}_{i} \cap V$ is a $G^{0}(\mathfrak{\xi})$-invariant subspace of $V$. Hence $\mathfrak{n}\left(E_{i}\right)$ and $\mathfrak{n}\left(E_{i}\right)$ are $A^{0}\left(\mathfrak{\zeta )}\right.$-invariant subspaces of $\zeta_{\text {. If }} E_{i}$ is $G(\mathfrak{g})$-invariant, then we can define the covariant systems $N\left(\mathfrak{P}_{i}\right)=N\left(E_{i}\right)$ and $M\left(\mathfrak{P}_{i}\right)=M\left(E_{i}\right)$ corresponding to $\mathfrak{P}_{i}$. Assume further that an isolated prime ideal $\mathfrak{P}_{i}$ is generated by elements of homogeneous degree 1, i.e., $\mathfrak{P}_{i}=\mathfrak{\Im}\left(E_{i}\right)$ for $E_{i}=\mathfrak{P}_{i} \cap V$. Then it follows that $M\left(\Re_{i}\right)(x)$ is the linear subspace of $D^{k}(x)$ spanned by the Monge characteristic elements corresponding to $\mathfrak{P}_{i}$.

REMARK 7.13. (1) $M\left(\Re_{i}\right)$ is called the Monge characteristic system corresponding to $\mathfrak{F}_{i}$ in [11].

(2) In [6], E. Cartan investigated "characteristic submanifolds" (multiplicites caracteristiques), which foliated each 3-dimensional integral manifold $N$ of $\left(R, D^{2}\right)$ satisfying $D^{2}(y)=T_{y}(N) \oplus \mathfrak{f}(x), y \in N$. The Monge characteristic elements are the candidates for the tangent spaces of such characteristic submanifolds. Not all Monge elements serve this purpose (cf. [6, p. 34]). Furthermore, the first covariant systems play a fundamental role in [5] and [6] for the further reduction of the equivalence and the integration problems of $\left(R ; D^{1}, D^{2}\right)$ (cf. Remark 5.18). We will take up these problems in the future papers. 


\title{
References
}

[1] R. L. Anderson and N. H. Ibragimov, Lie-Bäcklund transformations in applications, SIAM studies in Appl. Math. Ser. 1, SIAM, Philadelphia, 1979.

[2] V. I. Arnold, Characteristic class entering in quantization conditions, Functional Anal. Appl., 1 (1967), 1-13.

[ 3 ] A. V. Bäcklund, Ueber Flächentransformation, Ann. IX (1876), 297-320.

[4] C. Caratheodory, Calculus of Variations and Partial Differential Equations of the First order, Part I, Holden-Day, San Francisco-London-Amsterdam, 1965.

[5] E. Cartan, Les systèmes de pfaff à cinq variables et les équations aux dérivées partielles du second ordre, Ann. École Normale, 27 (1910), 109-192.

[6] E. Cartan, Sur les systèmes en involution d'équations aux dérivées partielles $\mathrm{du}$ second ordre à une fonction inconnue de trois variables indépendantes, Bull. Soc. Math. France, 39 (1911), 352-443.

[7] E. Cartan, Les systèmes différentielles extérieurs et leurs applications geometriques, Hermann, Paris, 1945.

[8] A. Giaro, A. Kumpera et C. Ruiz, Sur la lecture correcte d'un resultat d'Elie Cartan, C. R. Acad. Se. Paris, 287, Sér. A (1978), 241-244.

[@] H. Goldschmidt, Integrability criteria for systems of non-linear partial differential equations, J. Differential Geometry, 1 (1967), 269-307.

[10] K. Kakié, On involutive systems of partial differential equations in two independent variables, J. Fac. Sci. Univ. Tokyo, Sect. 1A, 21 (1974), 405-433.

[11] K. Kakié, Algebraic structures of characteristics in involutive systems of nonlinear partial differential equations, Publ. Res. Inst. Math. Sci., 13 (1977), 107-158.

[12] K. Kakié, Cauchy's characteristics of involutive systems of non-linear partial differential equations, Comment. Math. Univ. St. Paul. XXVIII-1 (1979), 8792.

[13] M. Kuranishi, On E. Cartan's prolongation theorem of exterior differential systems, Amer. J. Math., 79 (1957), 1-47.

[14] M. Kuranishi, Lectures on exterior differential systems, Tata Institute of Fundamental Research, Bombay, 1962.

[15] M. Kuranishi, Lectures on involutive systems of partial differential equations, Pub. Soc. Mat. São Paulo, 1967.

[16] T. Oshima and H. Komatsu, Partial Differential Equations of the First order, Kiso-Sûgaku, Analysis (II) iii, Iwanami Shoten, 1977, (in Japanese).

[17] R. Palais, A global formulation of the Lie theory of transformation gorups, Mem. Amer. Math. Soc. \#22, 1957.

[18] S. Sternberg, Lectures on Differential Geometry, Prentice-Hall, 1964.

[19] N. Tanaka, On differential systems, graded Lie algebras and pseudogroups, J. Math. Kyoto Univ., 10 (1970), 1-82.

[20] N. Tanaka, On pseudo-product structures and the geometrization of ordinary differential equations, to appear in Hokkaido Math. J..

[21] K. Yamaguchi, Geometrization of Jet bundles, to appear in Hokkaido Math. J..

\author{
DePARTMENT OF MATHEMATics \\ HOKKAIDO UNIVERSITY \\ SAPPORO 060, JAPAN
}

